\title{
A MULTIPLE CASE STUDY OF THE INTERAGENCY RELATIONSHIP BETWEEN SCHOOL ADMINISTRATORS AND LAW ENFORCEMENT PERSONNEL IN THE CREATION, IMPLEMENTATION, AND SUSTAINING OF SCHOOL EMERGENCY MANAGEMENT PLANS
}

\author{
by \\ Robert Lopez \\ Liberty University
}

\author{
A Dissertation Presented in Partial Fulfillment \\ Of the Requirements for the Degree \\ Doctor of Education
}

Liberty University

2018 


\title{
A MULTIPLE CASE STUDY OF THE INTERAGENCY RELATIONSHIP BETWEEN SCHOOL ADMINISTRATORS AND LAW ENFORCEMENT PERSONNEL IN THE CREATION, IMPLEMENTATION, AND SUSTAINING OF SCHOOL EMERGENCY MANAGEMENT PLANS \\ by Robert Lopez
}

\begin{abstract}
A Dissertation Presented in Partial Fulfillment
Of the Requirements for the Degree

Doctor of Education
\end{abstract}

Liberty University, Lynchburg, VA

2018

APPROVED BY:

James Swezey, Ed.D., Committee Chair

Russell Claxton, Ed.D., Committee Member

Patricia Cruz, Ed.D., Committee Member 


\begin{abstract}
The purpose of this qualitative multiple case study was to examine how the interorganizational relationship between school administrators and law enforcement personnel in the Midwest, South, and Southwest regions of the United States with diverse cultures, missions, and objectives to collaborate to create, implement, and sustain emergency management readiness. The theoretical concept that guided this study involved interorganizational theories as they relate to two or more organizations working together toward a common goal. This study used purposeful sampling of 12 participants from three school districts from the states of Alabama, New Mexico, and Illinois. A within-case and cross-case analysis along with interviews, documents, and artifacts, such as school safety plans, school safety drills, safety audits, and Safe School rubrics were analyzed as part of the data collection process and were used to investigate interorganizational relationships between schools and law enforcement in relation to school emergency management readiness. Results indicated that the creation of emergency management plans is primarily the work of district administrators. The implementation process included district administrators and building principals with minimal input of law enforcement personnel. However, the process of sustaining school emergency plans is a joint effort between school and law enforcement personnel. Analysis of data affirmed the importance of having an effective interorganizational relationship between school and law enforcement personnel in the context of school safety to create an effective emergency management plan in the school.

Keywords: Disaster, emergency management, interorganizational relationships, jurisdiction, school safety plans.
\end{abstract}




\section{Dedication}

I dedicate this study to my brothers and sisters of the military, law enforcement, and education who continually make the ultimate sacrifice to keep us safe and free in our daily lives. I am profoundly thankful for your dedication to service and country. May God bless all of you and your families.

"Blessed are the peacemakers, for they will be called children of God." Matthew 5:9 (NIV)

"Before I formed you in the womb I knew you, before you were born I set you apart." Jeremiah 1:5 (NIV) 


\section{Acknowledgments}

I thank God and my savior Jesus Christ for being there throughout my journey through life and this doctoral process.

I also thank my wife and family for their support throughout this doctoral process and understanding why I had to spend time away from family activities. The amount of cheerleading and support that I received from everyone over the last 3 years has been amazing. My mom moved on to be with Jesus some years ago but in my heart, I know that my accomplishment would have made her proud.

I am also thankful for God leading me to attend Liberty University. I have made some wonderful friends for life, including professors and fellow students. I want to thank my chair Dr. James Swezey in particular for mentoring and guiding me along the way. I appreciate the time he took for one-on-one prayer and friendly conversation outside of the dissertation journey. I also want to acknowledge my committee members Dr. Russell Claxton and Dr. Patricia Cruz who provided valuable insight and encouragement. Additionally, I would like to thank Dr. Cruz who has been with me through almost all of my coursework and dissertation process. Her advice and stories about her doctoral journey were immensely helpful.

I want to thank my fellow classmate Sherry for her friendship, prodding, encouragement, and laughter, which has made this journey much more bearable. 


\section{Table of Contents}

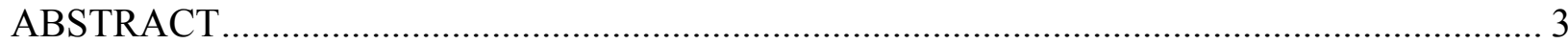

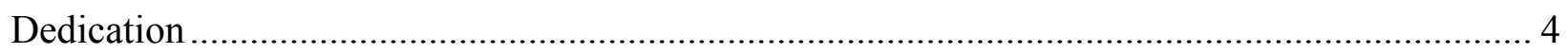

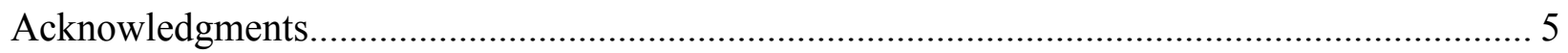

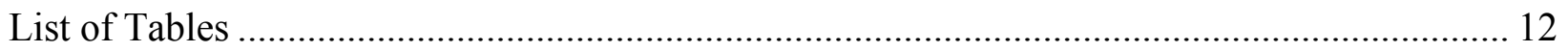

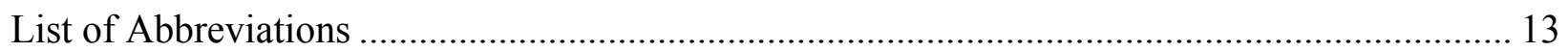

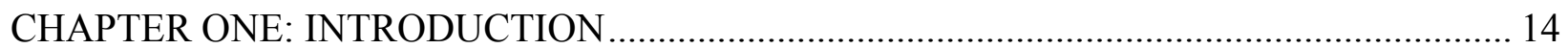

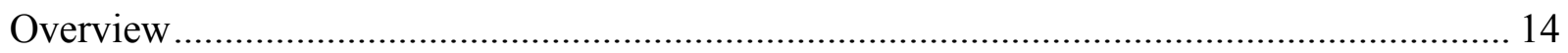

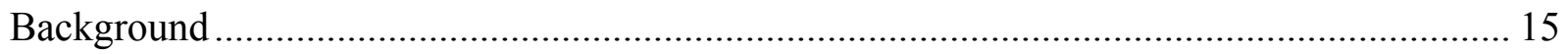

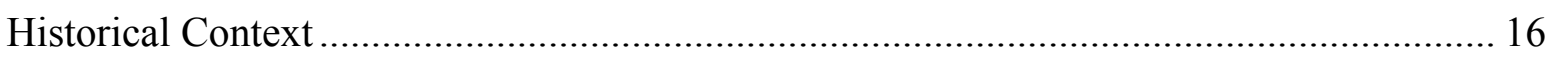

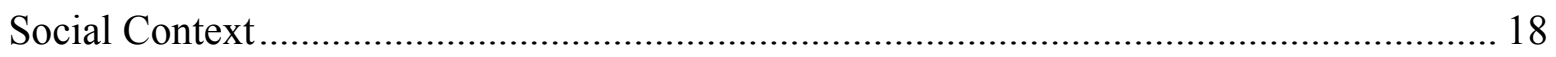

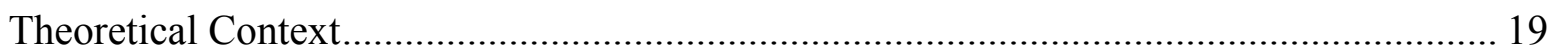

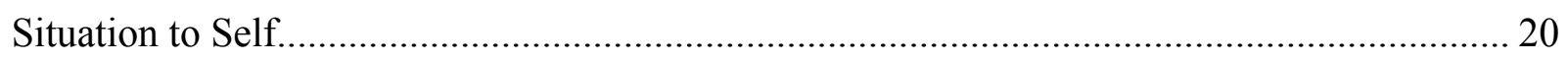

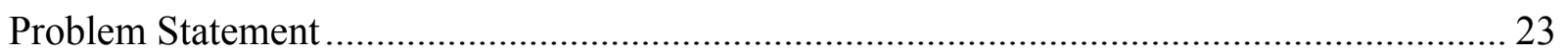

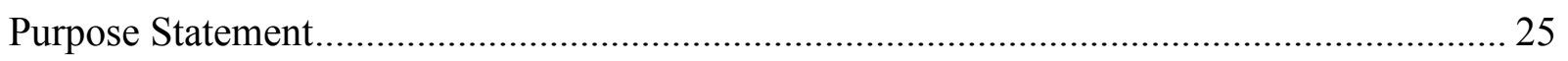

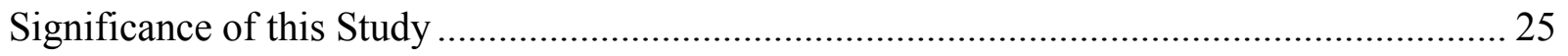

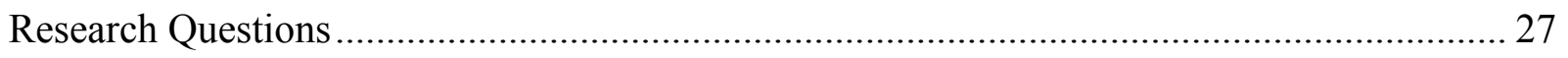

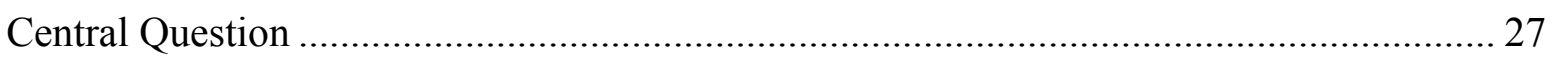

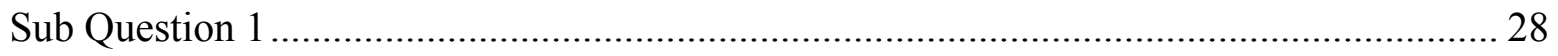

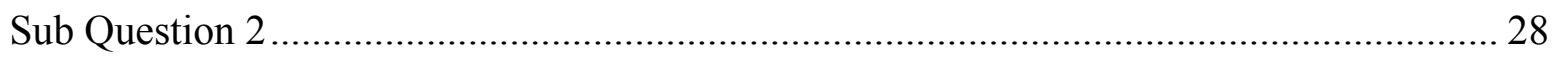

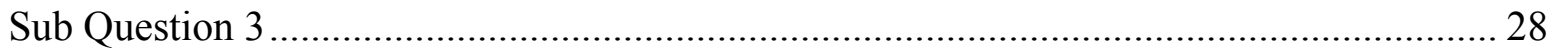

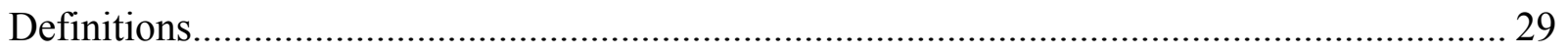




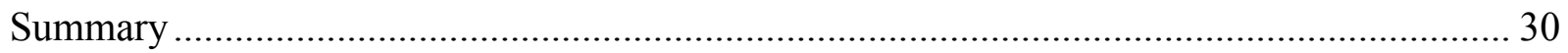

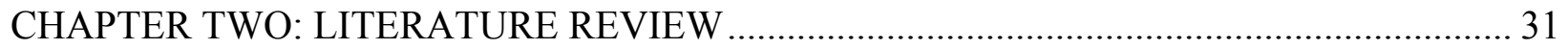

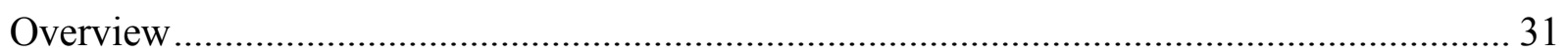

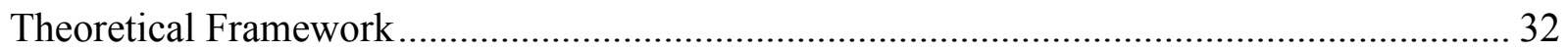

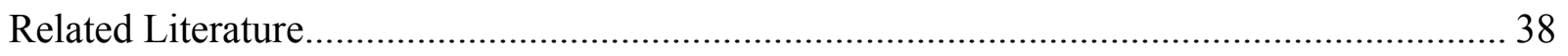

U. S. Department of Education School Emergency Operations Plans .................................. 38

International Versions of School Emergency Plans............................................................... 40

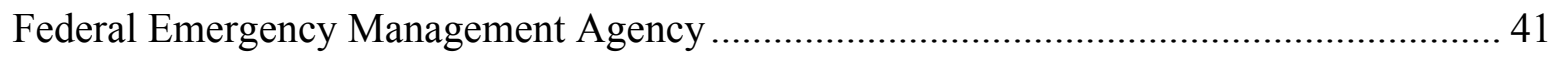

National Incident Management System (NIMS) ……………………………………..... 44

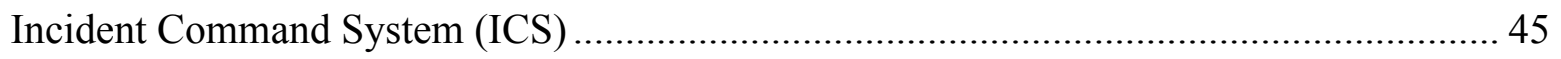

School Emergency Operations Plans ................................................................................ 45

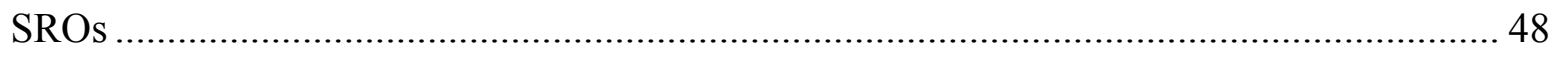

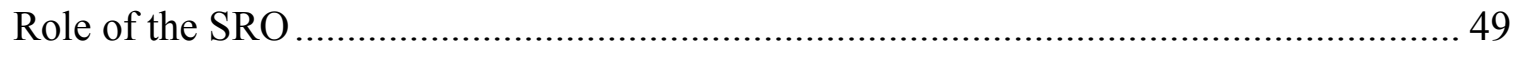

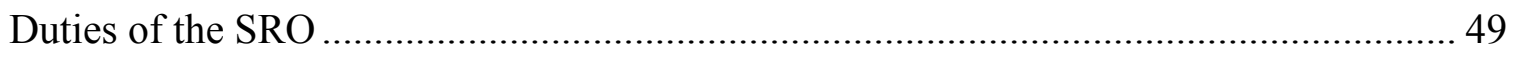

School Administrators, Teachers, Staff, and Student Views on SEOs ............................. 51

Role of School Administration .................................................................................... 52

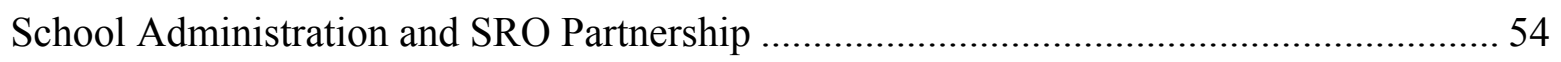

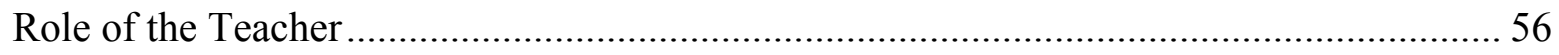

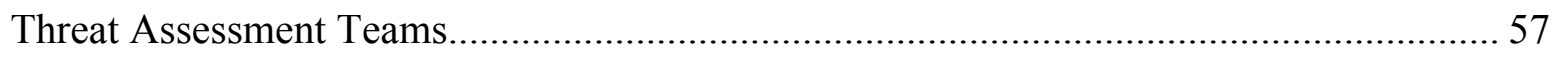

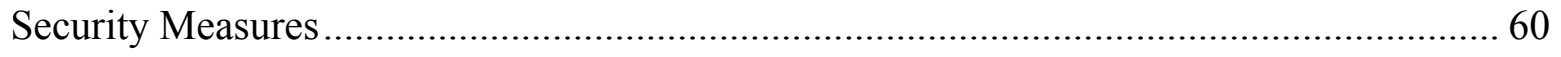

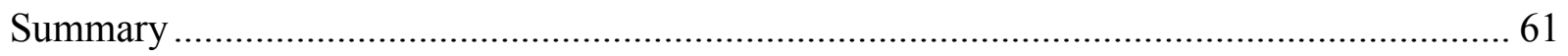

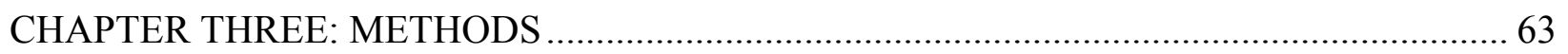

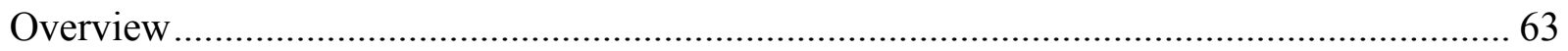




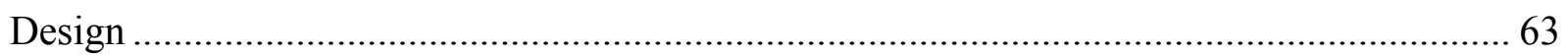

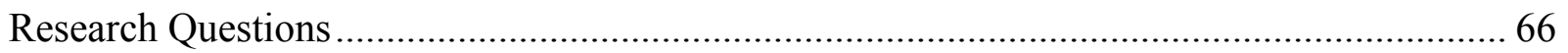

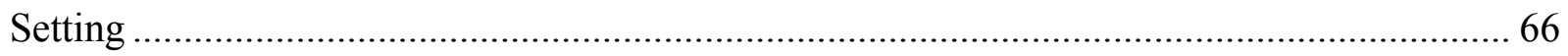

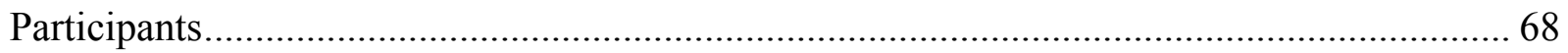

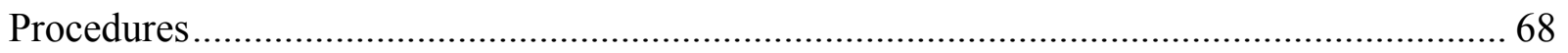

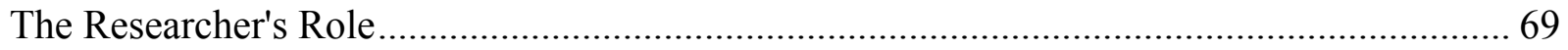

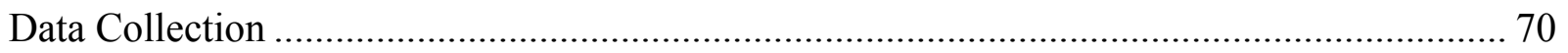

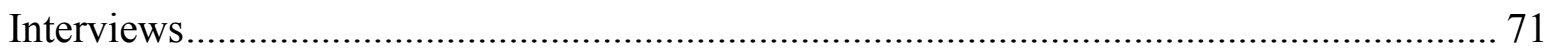

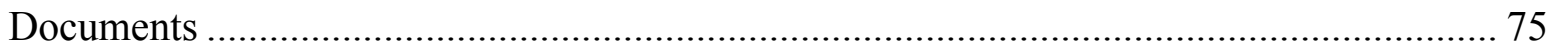

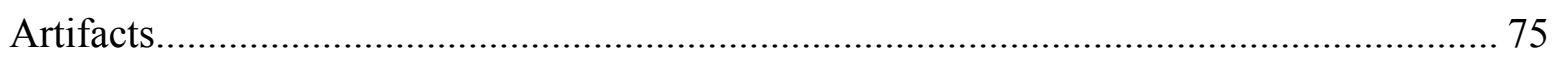

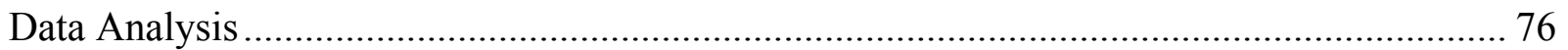

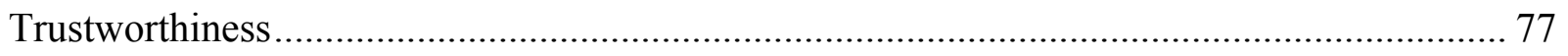

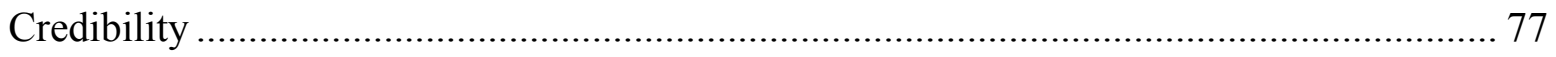

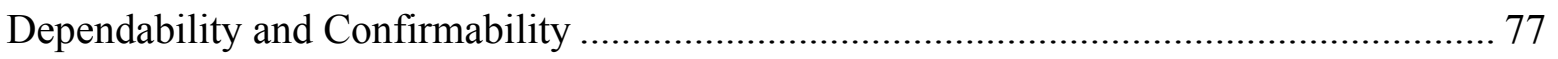

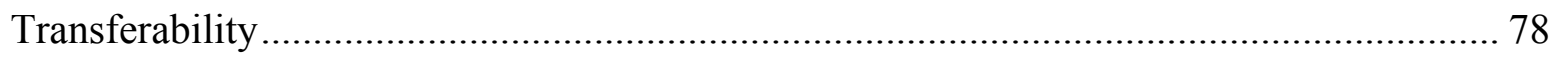

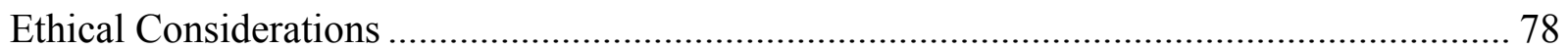

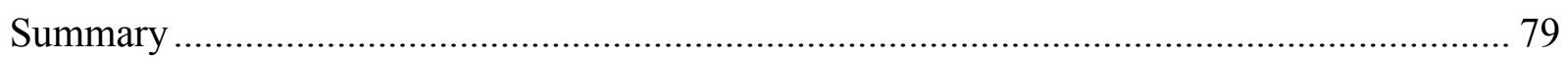

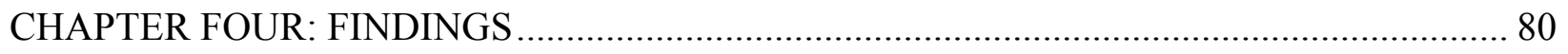

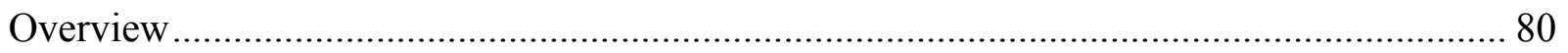

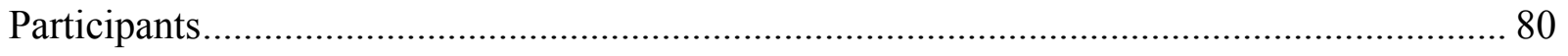

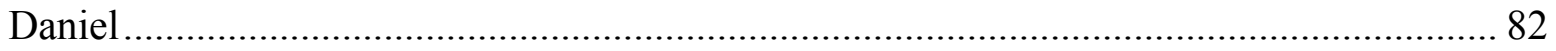

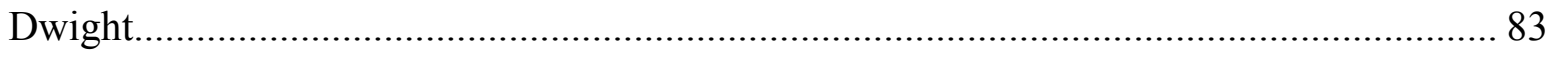

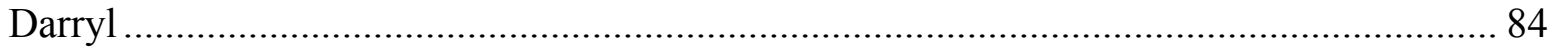




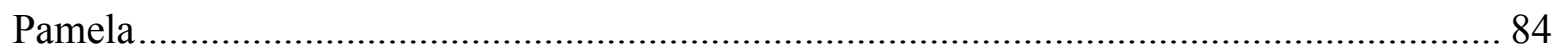

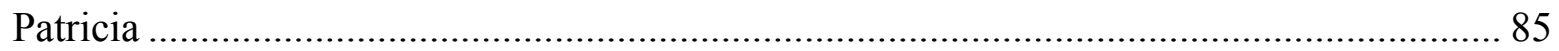

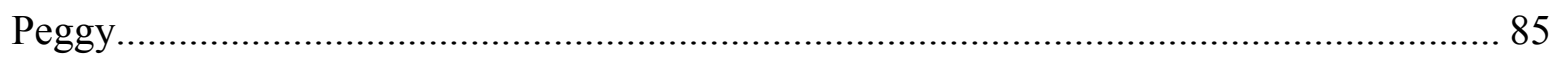

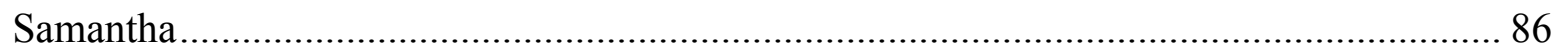

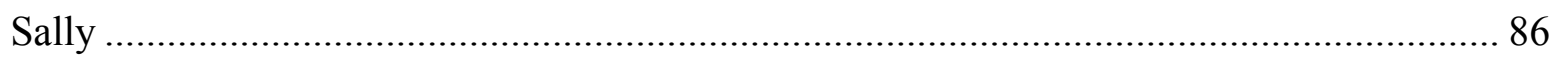

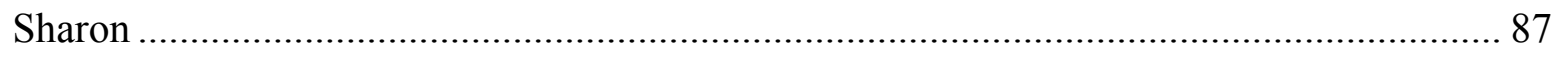

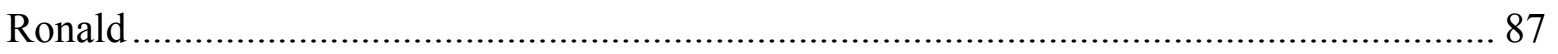

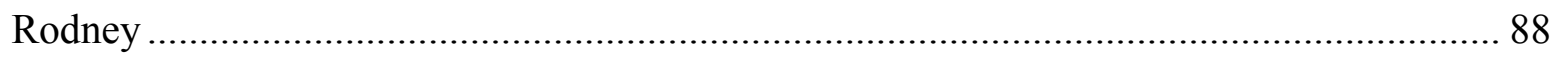

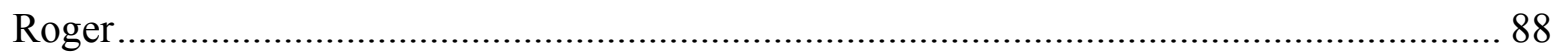

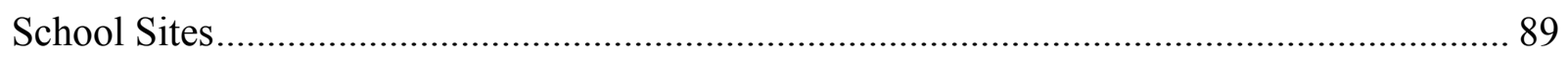

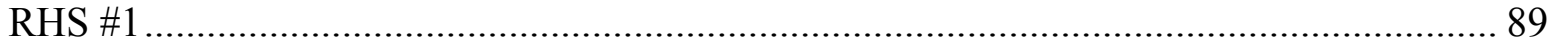

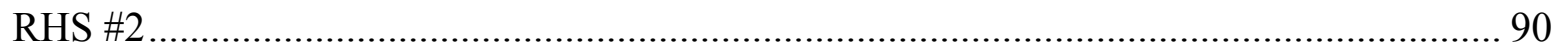

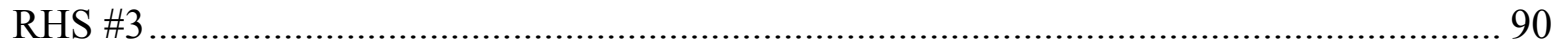

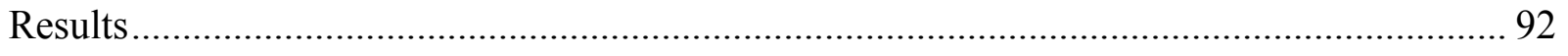

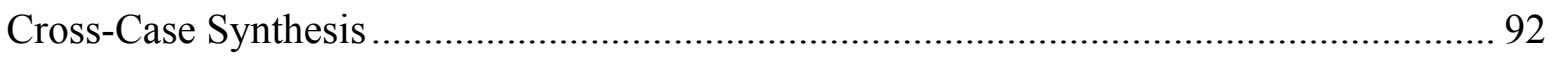

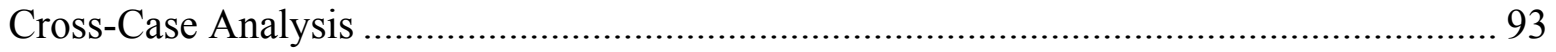

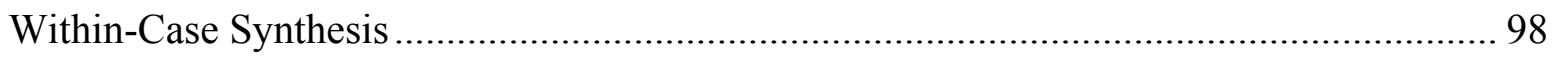

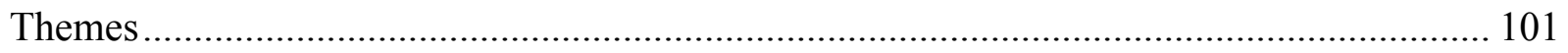

Theme One: Communication .............................................................................. 101

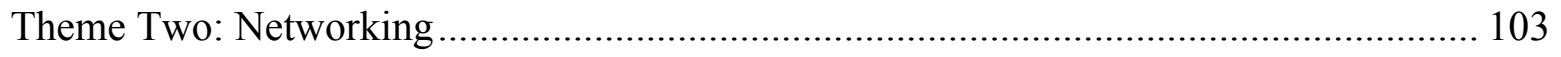

Theme Three: Mutual Understanding ................................................................ 105

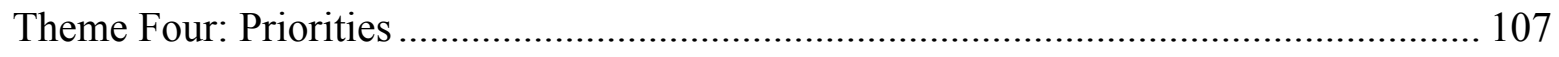

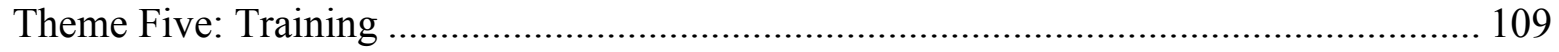


Answers to Research Questions 112 CQ: How Do School and Law Enforcement Personnel from the Midwest, South, and Southwest Regions of the U.S. Collaborate to Create, Implement, and Sustain School Emergency Management Readiness

SQ1: How Do Personnel from Schools and Law Enforcement Create School Emergency Management Plans 116

SQ2: How Do Personnel from Schools and Law Enforcement Implement School

Emergency Management Plans

SQ3: How Do Personnel from Schools and Law Enforcement Sustain School Emergency

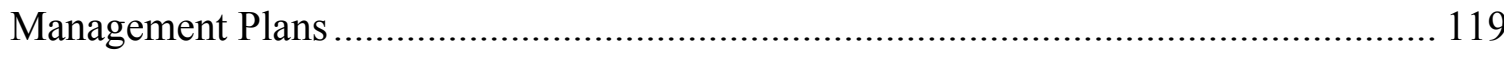

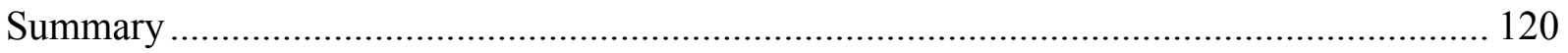

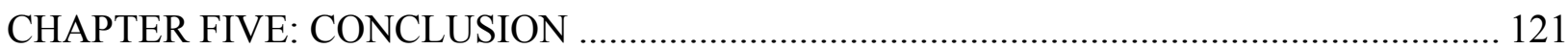

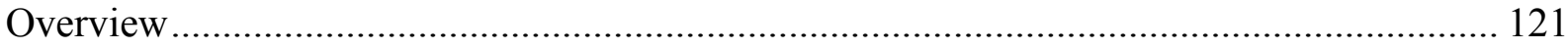

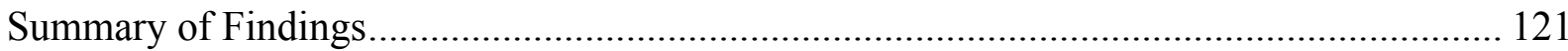

Central Research Question and Sub Questions....................................................... 122

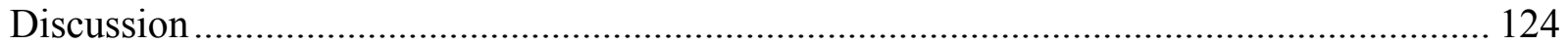

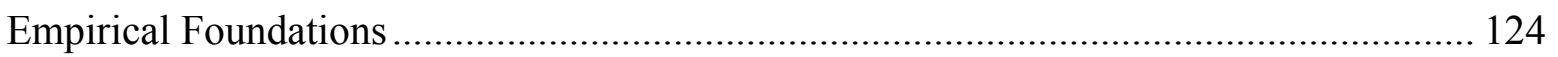

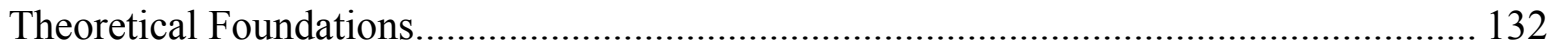

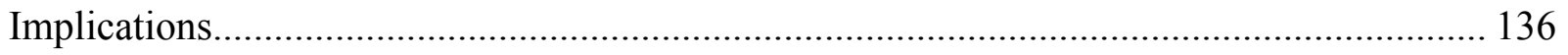

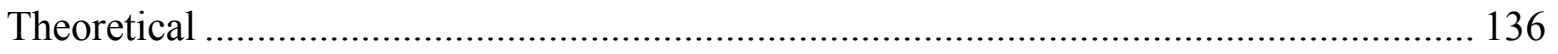

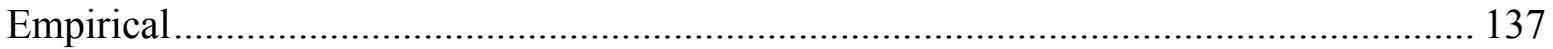

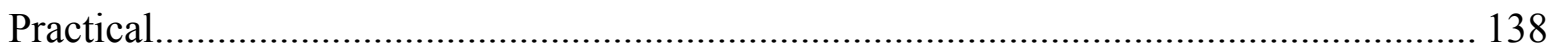

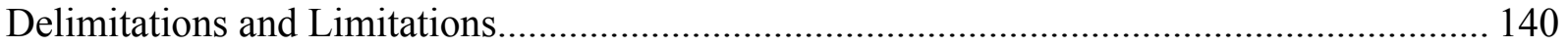


Recommendations for Future Research ..................................................................... 142

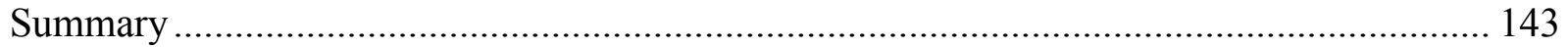

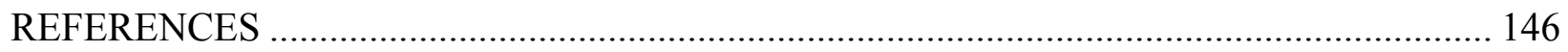

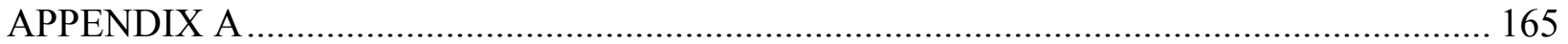

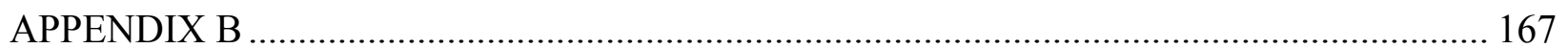

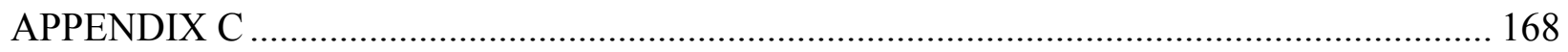

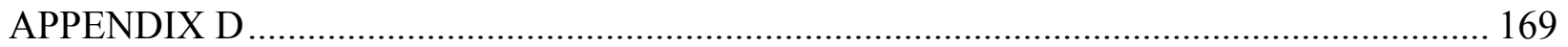

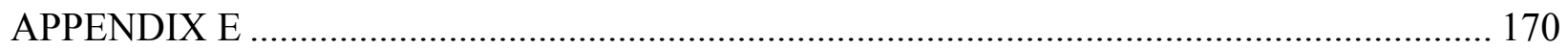




\section{List of Tables}

Table 1: Interview Questions ...................................................................................... 72

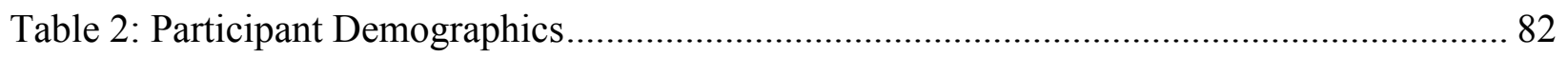




\section{List of Abbreviations}

Department of Homeland Security (DHS)

Federal Emergency Management Association (FEMA)

Incident Command Systems (ICS)

Law Enforcement Management and Administrative Statistics (LEMAS)

Memorandum of Understanding (MOU)

National Incident Management Systems (NIMS)

Non-Government Organizations (NGOs)

Post-Traumatic Stress Disorder (PTSD)

Region High School \# 1 (RHS \#1)

Region High School \# 2 (RHS \#2)

Region High School \# 3 (RHS \#3).

Secure Ammunition and Forearms Enforcement Act (SAFE)

Service Integration (SI)

State Emergency Management Systems (SEMS)

School Resource Officers (SROs) 


\section{CHAPTER ONE: INTRODUCTION}

\section{Overview}

The purpose of this chapter is to provide a framework for this multiple case study. Background information about the interorganizational relationships between school and law enforcement personnel regarding creating, implementing, and sustaining of school emergency management readiness for one rural and two suburban public, pre-k-12, school districts are examined. The planning, response, mitigation, and recovery methods of emergency management will be closely studied as each pertains to school emergency management. The purpose statement will explain the goal of the study, and an analysis of the importance of the study will be included. This chapter includes research questions that guided this study along with important definitions that provide a thorough understanding of school emergency management concepts.

Managing manmade, natural, and technical disasters is becoming increasingly important with the increase of conflicts, urbanization, and the potential for natural disasters. As a result, the majority of schools in the United States are creating school safety plans designed to aid in keeping students and staff safe (Kano, Ramirez, Ybarra, Frias, \& Bourque, 2007; Madfis, 2016). The U.S. Department of Education policies and some political leaders have strongly suggested that school districts collaborate with local first responders to develop school safety plans because all schools are vulnerable to threats, hazards, and disasters that could disrupt daily school operations (as cited in O’Toole \& Meier, 2011).

An all-hazards type of approach to school emergency readiness is needed because written plans alone are not enough to build resiliency in the event of a disaster (Hull, 2011; Perry \& Lindell, 2003). Several authors have explained that written plans without training exercises and 
collaboration with outside agencies can result in a poor response outcome (Brudney \& Gazley, 2009; Comfort, Ko, \& Zagorecki, 2004; Kapucu \& Hu, 2014). Recent studies have shown a lack of effective emergency planning and preparation in schools across the United States (Chrusciel, Wolf, Hansen, Rojek, \& Kaminski, 2015; Crawford \& Burns, 2015).

Most schools are directed to have some type of emergency plans and exercises; however, “...not all schools are uniformly prepared, trained, or resource equipped to respond to school emergencies" (Hull, 2011, p. 440). Additionally, schools with written sets of emergency plans that use a general template are not sufficient for an effective strategy. Written emergency plans need to be tailored according to potential hazards that each school may experience. Having written school safety plans is an important first step in having an effective school safety management system (Kano \& Bourque, 2007). It is equally important to sustain school emergency plans with regular training exercises with other agencies that may respond in the event of a crisis (Kano \& Bourque, 2007; Roher \& Warner, 2006).

One of the problems with disaster response during a major crisis is the potential conflict among stakeholders and a lack of collaboration that slows or thwarts the response process (Ansell \& Gash, 2007; Chikoto, Sadiq, \& Fordyce, 2013; Kapucu, Arslan, \& Demiroz, 2010). The issues with conflict and collaboration should have been worked out in advance with joint training exercises and regular meetings before an actual crisis situation (Chung, 2015; Roher \& Warner, 2006). Effective preparedness for disasters requires joint-training exercises, interagency meetings, and a collaborative decision-making process.

\section{Background}

Educational and law enforcement leaders have instituted a collection of strategies to prevent or diminish the harmful effects to schools that may occur due to man-made, natural, or 
technical disasters. An effective response is critical in the overall strategic process in the development of emergency management plans in all pre-k-12 schools (Kano \& Bourque, 2007). However, beyond the planning and preparation phase is the on-going collaborative relationship between schools and law enforcement that is critical in ensuring a smooth working relationship (Abbasi \& Kapucu, 2015; Amsler, 2016; Kapucu et al., 2010).

\section{Historical Context}

During the 1850s in the United States, a school building caught fire and because of a lack of fire safety preparation, several students died in the fire (Golway, 2003). As a result, schools across the U.S. began taking steps to prepare for emergencies by incorporating the use of regularly-scheduled fire drills (Heath, Pamela, Dean, \& Bingham, 2007). The practice of fire drills developed a smooth evacuation process thereby potentially saving lives.

During the 1950s and 1960s, several public and private schools began air raid drills in anticipation of a potential nuclear attack where students had to hide under their school desks (Heath et al., 2007). Similarly, many school districts located in regions that are known for certain types of natural disasters began to practice safety procedures specifically for those types of hazards (Federal Emergency Management Agencey [FEMA], 2016). For example, schools in California began preparing for earthquakes while schools in Florida began preparing for hurricanes.

Attacks on school and college campuses are not a new phenomenon. These incidents have been taking place for years. In 1927 one of the worst school attacks occurred in Bath County, Michigan that involved an irate school board member who carried out a series of bombings that killed 45 people, 38 of whom were children (Heath et al., 2007). Rocque (2012) noted that nearly 60 school shootings have taken place in American schools, with a rash of 
armed attacks occurring in the 1990s. This information shows that not all school attacks receive major media attention, which means that this problem may be worse than previously believed.

As a result of increased attacks, schools have intensified their security measures by adding School Resource Officers (SROs) and additional security devices on campuses across the country. One of the first known SRO programs started in Flint, Michigan in the late 1950s with the goal of bridging the gap between law enforcement and the local youth (Cray \& Weller, 2011). Not surprisingly, the program proved to be effective and grew extensively to where it is today (National Association of School Resource Officers [NASRO], n.d.).

A 2007 survey conducted by the Law Enforcement Management and Administrative Statistics (LEMAS) found there are more than 17,000 SROs assigned to public schools in the U.S. However, SROs are not required to register in any type of national database, but it is estimated by NASRO (n.d.) that approximately 17,000 are assigned to schools nationwide. Schools and law enforcement agencies must work together in developing comprehensive school emergency plans and making the effort to employ joint-agency exercises. However, collaboration between agencies for a variety of reasons has been problematic for decades (Aiken \& Hage, 1968; Reitan, 1998).

Agranoff (1991) reported that the movement to improve interagency relations in the human services began in the 1960s and 1970s because of the lack of effective combined efforts. During the 1960s and 1970s, independent agencies were working separately of other agencies and provided overlapping services. Extensive problems surfaced because of the lack of communication between the agencies. However, as agencies began to use Service Integration (SI) concepts, the federal government noticed the overall improvement in interagency 
relationships. The federal government has emphasized interagency program linkages, which has improved the ability of officials to manage comprehensive human services systems.

It is vital that a culture of collaboration be developed between educational institutions and law enforcement agencies with the mutual goal of developing effective emergency management systems for schools and districts (Hougland \& Sutton, 1978; Hull, 2011; Perry \& Lindell, 2003). Government organizations such as public schools and law enforcement agencies have a reputation of working together due to laws or policies of the jurisdiction that often require mutual participation (Aiken \& Hage, 1968; Houghland \& Sutton, 1978; Kapucu, Garayev, \& Wang, 2013).

\section{Social Context}

Sigmund Freud considered self-preservation to be a fundamental element of the human psyche (as cited in Khantzian \& Mack, 1989). Emergency management is a fundamental concept of self-preservation with the goal of keeping the self and property safe from potential harm (Quarantelli, 1997). Lindell and Perry (2012) posited that the stronger the environmental cue, such as sights and sounds of an impending hazard or disaster, the more likely that people will respond to protect themselves. Emergency management is built upon the concept that taking protective action in the form of preparation, response, mitigation, and recovery will lessen or prevent harm to persons or property (FEMA, 2016).

Disaster or hazard warnings may come from a variety of sources such as television, radio, newspapers, or computer technology, which in turn can create a protective reaction of the citizens (Lindell \& Perry, 2012). Quarantelli (1993) argued that the dominant reaction to a potential threat is not panic, but much the opposite. People normally choose a positive behavioral response. Nonetheless, those who have been previously exposed to a major disaster 
are more likely to react sooner and with a sense of urgency compared to those that have been through warnings of impending disaster but were not affected (Dillon, Tinsley, \& Burns, 2014; Weinstein, 1989).

In the case of a dangerous event, reactions will likely spark rapid and perhaps prolonged response until the public comes to terms with the crisis (Burns \& Slovik, 2007). Response to an adverse event will include first-responder agencies, government officials, and community leaders that will coordinate their efforts to enhance the recovery process (Burns \& Slovic, 2007). These types of coordinated efforts require practice before a crisis takes place. Waiting to coordinate first responder units in the midst of a chaotic, changing emergency will severely hamper their effectiveness. An effective response requires an extensive amount of planning, preparation, and collaborative efforts (Brudney \& Gazley, 2009; Kapucu \& Hu, 2014).

\section{Theoretical Context}

There is one theoretical concept that is closely related to emergency management responses and collaborative efforts among varied organizations that will guide this study. Interorganizational relationships are a significant component of emergency management and coordination. During a disaster or emergency event, several organizations must work together to ensure the safety of all persons involved. These organizations could consist of government organizations, non-government organizations (NGOs), and emergent organizations (Ansell, \& Gash, 2007; Kapucu et al., 2010).

For this study, the government organizations involved primarily consist of schools, law enforcement, fire departments, emergency medical teams, and emergency management. Nongovernment organizations are private agencies within the community that assist with the emergency management process. Emergent organizations are groups of volunteers that may be 
created ad-hoc during a crisis (Drabek, \& McEntire, 2003; Perry \& Lindell, 2003; Quarentelli, 1993). Drabek and McEntire (2003) described an emergent group as one that voluntarily reacts to assist others during a crisis.

The concept of interagency collaboration during the emergency management process is a fundamental factor in the success of any response to a disaster or hazardous situation, especially in a school setting (Kano \& Bourque, 2007). Bhandari, Owen, and Brooks (2014) described the major components of emergency response as adaptability, stability, stakeholder communication, and leadership as being the prominent features of emergency management. Communication and collaboration among independent organizations that have to work together during a time of crisis are enormously difficult tasks (Comfort et al., 2004; Kapucu et al., 2010). However, if the goal is to deliver reliable services in the event of a crisis, those involved in this dynamic relationship must work cohesively through a functioning interorganizational relationship (Gonzalez, 2010;

Reitan, 1998). Emergency management without systematic cooperation, joint training exercises, strategic planning, and proper communication among the stakeholders severely limits the chances of success in disaster response (Perry \& Lindell, 2003).

\section{Situation to Self}

My goal in conducting this study is to analyze the interorganizational relationships and emergency school management used in protecting students and staff members in the event of a manmade, natural, or technical disaster on or near a school campus. As an educator and parent, my hope and motivation is that this study will contribute to school emergency management readiness, which could potentially save lives. School campuses, both pre-k-12 and postsecondary institutions, are vulnerable to a multitude of potential problems including terrorist attacks and natural and technical disasters. The good news is that steps can be taken that will 
lesson or prevent the loss of lives and property. As a former law enforcement officer and current school administrator, I hope to see school districts understand the potential for a crisis and take necessary steps to lessen or prevent any harm to students or staff. It is my hope that this study will contribute to the current studies associated with school emergency management.

During my career, I served in the United States Marine Corps as a military police officer. After completing my contract, I went to work with the Los Angeles Police Department and later moved to Georgia where I worked as a SRO and criminal justice instructor. I completed my law enforcement career in New Mexico where I served as the Director of Public Safety.

My career in education includes stints as a full-time college professor and director of criminal justice programs to my current position as a district crisis and safety director for a school district. For the past five years, I have worked in high school administration, which has sparked my interest in researching the collaborative efforts between schools and law enforcement agency personnel for the purpose of developing school emergency management plans.

Experiences in education and law enforcement have led me to use the interpretive framework of pragmatism. In my opinion, there is not a single answer to the question of instituting cooperative efforts in an interagency task of developing emergency plans for schools. As stated by Creswell (2013), truth is dependent on what is working at a specific point in time, and the search for the current truth will require multiple sources of data from social, political, and historical sources.

There are four philosophical assumptions discussed in Creswell (2013): ontological, epistemological, axiological, and methodological. Creswell asserted that ontological is the belief of a concept that is derived from multiple realties that are garnered from multiple forms of evidence. Ontological is based on what an individual believes based on themes using words and 
perspectives of different individuals. Epistemological assumption is the development of explaining how a people know what they believe about a reality. Past experiences create an explanation of a person's processing of an idea to form a belief. Accumulation of knowledge in a particular study is based on the observations and participation from individuals that are involved in the study. Axiological assumption is based upon the abounding values of the nature of the study. The author argued that research is formed from the values that shape the explanation with the added interpretation of the researcher's findings. Information from the study is compiled, investigated, and analyzed to provide an interpretation from the mindset of the researcher. Methodology is the path chosen by the researcher to formulate deeper insight and knowledge of the data from the study. Researchers use certain processes or methods, which will dictate or form a pattern of the study.

The philosophical assumption that will most closely dictate my approach to this study will be epistemological that follows two frameworks: social constructivism and pragmatism. First, social constructivism, according to Creswell (2013), is a co-constructed reality between the researcher and the participants whose views are shaped by individual experiences. Both the researcher and the participants will have had unique experiences, philosophies, and visions about the concept of school emergency management depending on the experiences and roles played by each individual. A pattern or meaning will be developed through the interviews and analysis rather than beginning with a particular theory and attempting to prove it. In this study, meaning will be developed through the interpretive lens of individuals and interactions that will take place during the study. In other words, a theoretical concept will develop naturally after all data are gathered and analyzed. 
The goal of the research in this multiple case study is to "... rely as much as possible on the participants' views of the situation" (Creswell, 2013, pp. 24-25). Following Creswell's suggestions, patterns and meanings will appear as the individuals share their beliefs and experiences in relation to school emergency readiness. I attempted to remove my biases and experiences as much as possible and relied on evidence gathered from qualitative narratives of the participants so as not to influence the study with my personal beliefs. Other than straightforward demographic and historical queries, open-ended questioning was used to obtain the participants' perspectives from their life setting. One of the objectives in this study was to allow the participants to create an individual essence of their interpretation through story telling.

Second, the framework of pragmatism is the identity of a reality, which is gained through the analysis of using both deductive and inductive reasoning (Creswell, 2013). Similar to the philosophical assumption of social constructivism described by the author, pragmatism does not begin with a particular theory in mind. An interpretation of the information is developed from the outcomes and the problem being investigated. Creswell described pragmatism as the opportunity to use varied systems of philosophy instead of relying on a single concept. Answering the research question on how school and law enforcement personnel with diverse cultures, missions, and objectives collaborate to create, implement, and sustain school emergency management readiness required a variety of qualitative approaches. For this study, pragmatism was important because it allows the use of a multiple approaches to collect and analyze data that will provide meaningful patterns and themes.

\section{Problem Statement}

Manmade, natural, or technical disasters can occur near or on any school campus in the United States. Planning, preparing, mitigation, and ultimate recovery for these types of 
emergencies are crucial to potentially limiting or preventing harm to students and staff at a time of the crisis. Planning, preparation, mitigation, and recovery are all emergency management phases that are of equal importance in comprehensive school safety emergency readiness.

As part of the planning and preparation process, it is imperative that school administration and emergency first-responders collaborate in developing campus safety emergency management plans. School administration and local first responder agencies need to train and practice together to become familiar with the school buildings and expose potential hazardous shortcomings prior to an emergency event (Avery, Graham, \& Park, 2016; Bondu \& Beier, 2015 Chrusciel et al., 2015).

To meet minimum standards, school safety plans should include pertinent information from the Federal Emergency Management Agency (FEMA), the National Incident Management System (NIMS), the State Emergency Management System (SEMS), Incident Command Systems (ICS), and the Guide for Developing High-Quality School Emergency Operations Plans through FEMA and the U.S. Department of Education as guidelines. Additionally, plans must be uniquely suited to individual schools, which includes a map of the building and surrounding neighborhood to identify entry and egress points (Bradford, \& Wilson, 2013; Shiwaku, 2014; Wang, 2016; Watson, Loffredo, \& McKee, 2011).

The problem is attempting to find out how school and law enforcement personnel interact to create, implement, and sustain school emergency management safety plans in three high schools located in the Midwest, South, and Southwestern regions in the United States. This type of information is necessary to identify school needs and best practices in ensuring the safety of schools. 


\section{Purpose Statement}

The purpose of this qualitative multiple case study was to examine the interorganizational relationship between school administrators and law enforcement personnel in creating, implementing, and sustaining emergency management readiness of one rural and two suburban, pre-k-12 school districts in the Midwest, South, and Southwestern regions in the United States. At this stage of the research, the interorganizational relationship is defined as the integration of multiple agencies and jurisdictions that form a smoothly-functioning, interorganizational response system during routine operations and under the urgent, chaotic conditions of a full-scale disaster (Comfort \& Kapucu, 2006).

The initial process and follow through of school emergency plan development requires a multi-jurisdictional coordinated effort. Theories of interorganizational relationships assisted and guided this study in examining the relationships between multiple agencies. The majority of emergency response organizations previously mentioned includes local, regional, state, and federal government agencies.

\section{Significance of this Study}

This qualitative multiple case study hopefully provides an additional perspective to current studies that pertain to pre-k-12 school emergency management plans, readiness, and the collaborative interagency efforts between schools and law enforcement agencies. School administration, law enforcement personnel, first responders, and agencies involved in interagency relationships may find this study useful. An analysis will be provided to demonstrate a unique insight into educational and law enforcement systems collaborating to build a comprehensive approach to school emergency management readiness (Kapucu \& Hu, 2014; Provan \& Milward, 1995; Waugh, 2003). The choice to limit this study to schools and law 
enforcement was due to the partnerships currently formed through Memorandum of Understanding (MOU) and SRO programs across the United States.

Currently, there is a limited amount of research available on disaster preparation of emergency management for schools and the collaborative efforts between stakeholder agencies, namely law enforcement (Kano \& Bourque, 2008; Kapucu \& Hu, 2014; Cray \& Weller, 2011). One of the problems, according to Brown (2006), is determining if two different cultures, in this case schools and law enforcement, can effectively work together. This study looked into the dynamics of two significantly different systems, school and law enforcement personnel, and their ability to form a school emergency management partnership.

The theory that is integrated within this study was the theory of interorganizational relationships (Aiken \& Hage, 1968; Bhandari et al., 2014; Hougland \& Sutton, 1978; Perry \& Lindell, 2003; Reitan, 1998). This study hopefully adds to the current literature on school emergency management plans by contributing a distinct viewpoint from schools and law enforcement personnel networking toward a common goal (Steelman \& Mandell, 2011).

This study may assist schools and law enforcement in preparing emergency management plans to create an effective response in crisis situations. Schools and law enforcement personnel have distinct cultures, missions, and objectives but also possess a common goal of keeping students and staff members safe. With the current state of potential terrorist acts, school shootings, and natural disasters, the need for effective and efficient school emergency management plans is at an all-time high (Kano \& Bourque, 2007).

The U.S. Department of Education (2013) strongly suggested that all schools implement school emergency plans, which is a step in the right direction; however, written plans alone do not create comprehensive readiness in the event of a disaster. According to Comfort and Kapucu 
(2006), written plans must be developed using a collaborative effort among responding agencies to ensure a coordinated reaction in a chaotic, dynamic environment. In taking emergency management readiness to a more effective level, the plans must include regular joint training exercises, meetings among the stakeholders, and opportunities to develop familiarity and trust among the network (Kapucu, 2006b; Provan \& Milward, 2001).

Several authors have discussed the concept of shared risk, frequent interaction, and a common set of goals as imperative in creating effective networks in emergency management (Brudney \& Gazley; Kapucu, 2006b; Waugh \& Streib, 2006). This study should add a different perspective of the network dynamic between schools and law enforcement personnel in the creation, implementation, and sustaining of school emergency management plans.

\section{Research Questions}

An effective interorganizational relationship between school administration and law enforcement personnel is an essential component of developing a comprehensive school emergency management plan. Currently, a limited amount of research indicates that comprehensive school emergency management is not being implemented consistently across the country (Kano \& Bourque, 2008). The following research question will be used to examine the readiness of school emergency management of three distinct school districts:

\section{Central Question}

How do school and law enforcement personnel in the Midwest, South, and Southwestern regions in the United States with diverse cultures, missions, and objectives collaborate to create, implement, and sustain emergency management readiness? This study is an investigation into the dynamic relationship between school and law enforcement personnel and their ability to work together toward the goal of school emergency management readiness. 


\section{Sub Question 1}

How do personnel from schools and law enforcement create school emergency plans? School emergency management readiness has been a requirement of schools in the United States for decades (Cray \& Weller, 2011). School emergency plans should be a joint effort between schools and law enforcement as each organization can bring their unique experiences and expertise (Coon \& Travis, 2012). This question will provide insight into the process used to develop school emergency plans.

\section{Sub Question 2}

How do personnel from schools and law enforcement implement school emergency plans? Once school emergency management plans have been created, the next step will be implementation, which requires networking between stakeholders to ensure the effectiveness of school safety plans (Sowa, 2008). This question is an opportunity to investigate the mechanism of taking written plans and putting them into practice.

\section{Sub Question 3}

How do personnel from schools and law enforcement sustain school emergency plans? While having effective written plans is a critical component of school emergency management, sustaining the plans requires continual collaboration, drills, exercises, and evaluation (Trump, 2011). This final sub-question will provide insight into the precise procedures that schools and law enforcement use to take written plans and put them into physical form through drills, exercises, and evaluation.

The research questions set the groundwork of this qualitative multiple case study. For this study, the research questions were taken from literary research while attempting to fill the gaps in the literature. The central question will seek to answer the phenomena of how two 
distinct organizations can collaborate to obtain the common goal of school emergency management readiness.

\section{Definitions}

1. Disaster - A disaster is a sudden, calamitous event that seriously disrupts the functioning of a community or society and causes human, material, and economic or environmental losses that exceed the community's or society's ability to cope using its own resources (Drabek, 1970).

2. Interorganizational relationships - Interorganizational relationships are the joint cooperation with other organizations (Hougland \& Sutton, 1978).

3. Jurisdiction - Jurisdiction is the geographical area over which authority extends, legal authority, and the authority to hear and determine causes of action (U.S. Department of Education, 2013).

4. Memorandum of Understanding - A memorandum of understanding is an instrument used to build mutual respect and trust between partners while delineating specific roles and responsibilities of the partnering agencies (COPS Office, 2013).

5. Mitigation - Mitigation is the capability necessary to eliminate or reduce the loss of life and property damage by lessening the impact of an event or emergency (U.S. Department of Education, 2013).

6. Prevention - Prevention is the ability to avoid, deter, or stop imminent crime or a threatened or actual mass casualty incident (U.S. Department of Education, 2013).

7. Protection - Protection is the ability to secure schools against acts of violence and manmade or natural disasters (U.S. Department of Education, 2013). 
8. Recovery - Recovery is the capability necessary to assist schools affected by an event or emergency in restoring the learning environment (U.S. Department of Education, 2013).

9. Response - Response is the capability necessary to stabilize an emergency once it has already happened or is certain to happen in an unpreventable way (U.S. Department of Education, 2013).

\section{Summary}

Emergency management for American schools is a top priority because of the number of attacks on school campuses, the potential for terrorist attacks, and the likelihood of a natural or technical disaster occurring on or near school campuses. Not every school can be prepared for every contingency; however, it is still the responsibility of school leaders to make every effort to ensure the safety of students and staff. As part of that preparation process, schools and first responding agencies must use a coordinated bid to ensure that prevention, protection, mitigation, response, and recovery have been thoroughly evaluated, practiced, and applied.

This study intended to be an avenue to evaluate the current emergency readiness by examining the emergency management plans of one rural and two suburban pre-k-12 schools that employ the use of an SRO in Alabama, Illinois, and New Mexico. Additionally, this study may also serve as a guide for other educational institutions to use in examining and improving their current emergency management plans and interagency partnerships. 


\section{CHAPTER TWO: LITERATURE REVIEW}

\section{Overview}

School emergency management has been a challenge for decades. Because of the potential for natural disasters to occur anywhere in the world and oftentimes without warning, it is crucial for schools to employ and practice an all-hazards approach to safety. With the increase in terrorist activity and school attacks, it is becoming increasingly difficult to provide a safe environment for staff and students. School emergency management plans and templates are available through federal and state guidelines to assist schools in creating a safe school environment. However, research has shown that school emergency plans alone may not prove effective enough in diminishing or preventing the harmful effects of a calamity (Allen, Will, Brennon, \& Poirer, 2010; Kano et al., 2007; Roher \& Warner, 2006).

An all-hazards approach with multi-jurisdictional collaboration in school emergency planning is imperative to create a safe school environment. An all-hazards or comprehensive approach to school emergency management readiness includes planning, preparation, response, mitigation and recovery methods. Research in the area of interorganizational relationships between law enforcement and school administration in school emergency management is lacking (Cray \& Weller, 2011; Kano \& Bourque, 2008; Kapucu \& Hu, 2014). This study examined the areas of interorganizational relationships between school administration and law enforcement personnel as it relates to school emergency management.

This chapter contains the theoretical framework and related literature section that will guide this study. The literature review includes interorganizational theory, history of school emergency planning, federal and state guidelines for school emergency planning, international versions of school emergency management, and the roles of law enforcement and school 
personnel in the school emergency planning readiness context. Lastly, this chapter concludes with an overview of threat assessment teams, security measures, and a summary.

\section{Theoretical Framework}

The coordination of agencies has been and will continue to be problematic unless the organizations involved agree to collaborate toward a common goal (Aiken \& Hage, 1968; Coon \& Travis, 2012; Hougland \& Sutton, 1978; Kapucu, 2006a). There are multiple conflicting definitions and no clear solution that can provide a framework for analyzing the problems associated with interorganizational relationships (McGuire, \& Silvia, 2010; Reitan, 1998). As stated by Reitan (1998), one of the most pressing issues is the coordination of agencies, which is not a common occurrence among organizations for a variety of reasons. Aiken and Hage (1968) relayed that the greater the number of joint programs, the greater number of problems associated with interorganizational decision-making.

Multiple organizations do not have common goals, missions, policies, and interests that create a chasm in the relationship (Quarentelli, 1997). However, the likelihood of an individual entity successfully operating in a small or large-scale disaster is unlikely and dangerous. Each type of disaster brings its own unique challenges and obstacles that cannot be predicted but must be overcome with the assistance of other organizations (Perry \& Lindell, 2003; Waugh \& Streib, 2006). Some of the obstacles associated with multiple organizations combining their efforts must be facilitated to provide effective responses to emergency settings.

Interorganizational relationships between schools and law enforcement agencies in this study focused on the obstacles and difficulties that are presented when separate organizations endeavor to work together effectively. In developing interorganizational relationships, each organization must understand the others' missions, structures, styles of operation, capabilities, 
and limitations (Perry \& Lindell, 2003; Schnobrich-Davis \& Terrill, 2010). Stakeholders involved in emergency management have distinct roles and responsibilities; however, the importance of overcoming the disparity of each other's' roles can make the difference in an effective response (Chung, 2015; Coon \& Travis, 2012).

Organizational culture, missions, and objectives are unique to schools and differ significantly from law enforcement agencies, which may create obstacles to interorganizational effectiveness (Jackson, 2002). For example, the predominant mission of law enforcement is to protect and serve the community, whereas pre-k-12 schools have the mission of preparing students to become productive members of society (Coon \& Travis, 2012). The individual roles of law enforcement officers and teachers display significant differences as well. Paoline (2003) posited that police officers must work with an air of authority, which is ingrained in preparing officers from the police academy through the field training officer process. Police officers are described as distrustful of the general public, and decisions they make are based on potential threats; however, they are loyal to their own group because of the similar lifestyles (Blumenstein, Fridell, \& Jones, 2012; Lambert \& Steinke, 2015; Paoline, 2003). On the other hand, educators are groomed to be mentors and enlighten students in preparing them for life after high school (Blackaby, \& Blackaby, 2011). Mission and objectives among educators and law enforcement are varied, which creates a natural chasm that can be conquered by using a deliberative and multilateral process in creating an effective interorganizational relationship (Ansell \& Gash, 2007; Cray \& Weller, 2011).

One of the first steps in creating a collaborative atmosphere between organizations is to develop mutual trust and respect through frequent interactions (Ansell \& Gash, 2007; Kapucu et al., 2010). These joint interactions would be in the form of regular emergency preparation 
meetings, joint training exercises, or non-business-related functions. While the importance of emergency management training cannot be overstated, non-business-related meetings allow for opportunities for personnel to interact in a relaxed environment (Kapucu et al., 2010).

Social networking among individuals from separate organizations that are part of a partnership is essential in developing trust (Brudney \& Gazley, 2009; Kapucu, 2006b; Provan \& Milward, 2001). Sowa (2008) noted that working and interacting together influences how the two organizations will effectively progress over time, which could strengthen the trust factor within the interorganizational relationship. Trust and fostering relationships, according to Kapucu and $\mathrm{Hu}$ (2014), is essential in building an effective emergency management network.

Kapucu and Sadiqu (2016) explained that sharing information and developing a willingness to collaborate through mutual trust and understanding is critical to developing an effective relationship among differing agencies. Trustworthiness is based on the extent to which each organization can depend on its honor and commitment to each other (Provan et al., 2007). Therefore, it is imperative for the organizations to engage in a formal, collective, mutual goal setting process (Ansell \& Gash, 2007). Trust is crucial for an effective response during the chaos caused by an extreme event (Brudney \& Gazely, 2009). However, there is little to no time to develop an effective, trusting relationship in the midst of an emergency. Building trust is an ongoing, sustainable endeavor (Kapucu, 2006b; Kapucu, et al., 2010; McGuire \& Silvia, 2010).

Recent studies have shown that building and sustaining an effective collaborative network is crucial in effective emergency management (Kapucu \& Hu, 2014). Collaboration is a process of facilitating and operating in a multi-organizational structure to achieve a purposeful arrangement (McGuire, 2006a). Brudney and Gazley (2009) added that studies of collaborative activity both in and out of the emergency management field have found that the characteristics in 
strong partnerships include frequent interaction, shared experiences, and goals and expectations. Developing and engaging in effective collaborative networking and relationship building between organizations does not happen by chance; it takes time and hard work (McGuire \& Silvia, 2010).

Rapid technology, scarce resources, and rising interorganizational interdependency are creating reasons for new levels of collaboration among public agencies (Thomson \& Perry, 2006). Interorganizational relationships are close, long-term, mutually-beneficial agreements between two or more partners with shared objectives (Casey, 2007). These types of partnerships are usually developed when separate organizations find that working together is beneficial to both organizations.

Emergency management starts at the local level and must first use its own resources and strengths for mitigating, preparing, responding, and recovering from a disaster (Kapucu \& Van Wart, 2008). However, disasters have complexities that may require interorganizational cooperation because the dynamics involved in emergency response often exceed a single jurisdiction's ability (McGuire \& Silvia, 2010). Emergency management is a type of function that frequently crosses jurisdictional boundaries because the demands can easily overload local and state resources (Casey, 2007). Agencies must be prepared to seek additional assistance because of the dynamic changes that can take place during a disaster.

Emergency management response in a school related disaster is challenging because most school personnel are not trained to react as first-responders, but school staff will often be the first on scene (Bobo, Hallenbeck, \& Robinson, 2003; Kano \& Bourque, 2007). Additionally, formal first-responders are frequently delayed in reaching the disaster area, and the layperson initiates first-aid and search and rescue attempts (Kano \& Bourque, 2007b). The majority of schools in 
the United States are not tasked with specifically training school personnel to act as a professional first-responder (Kano \& Bourque, 2007a). For this reason, it is imperative that schools develop an interorganizational relationship with first-responder agencies, namely police departments, in developing, implementing, and sustaining school emergency management readiness.

One of the formal steps in creating an interorganizational relationship with a law enforcement agency that provides law enforcement services within the jurisdiction is through a memorandum of understanding (MOU). An MOU is an agreement between agencies that spells out the responsibilities of each agency that assists in alleviating any misunderstandings of each other's roles in the agreement (Burling \& Hyle, 1997; Schnobrich-Davis, \& Terrill, 2010). Cray and Weller (2011) added that effective SRO programs must have an MOU in place that contains the goals of reducing school violence and the steps to provide a safe learning environment. Furthermore, the MOU must provide detailed information on the roles and expectations of each position within the organizations that will assist in improving collaborative efforts of the schools and law enforcement agencies (Burling \& Hyle, 1997; Cray \& Weller, 2011). Basic information in an MOU should include terms of agreements, sites of assignments, regular duty hours, SRO training requirements, employment status of the SRO, vacation and summer hours, appointment of the SRO, and termination of the agreement. All stakeholders should annually review the MOU to ensure understanding of roles and to make any necessary adjustments (Cray \& Weller, 2011).

While it may be obvious that both school officials and members of law enforcement organizations value school safety, the varied backgrounds, educational attainment, and training bring vastly different perspectives in achieving school safety. A lack of understanding each 
other's expectations and goals creates problems unless these differences are resolved (Coon \& Travis, 2012). Brown (2006) questioned if school and law enforcement relations with two completely different cultures can effectively work together on a consistent basis. Jackson (2002) added that some of the obstacles with the SRO programs are due to the cultural differences between law enforcement officers and educators. Collaboration is often difficult because of the closed climate associated with law enforcement and the open environment of schools. Moreover, Jackson (2002) observed that SROs are primarily concerned with school safety, whereas educators support the ideal of a supportive learning environment that is focused on educational achievement.

The SRO and school administration partnership is a complex issue that has competing orientations, practices, and assumptions regarding the handling of discipline and safety within the school environment (Cray \& Weller, 2011). Coon and Travis (2012) purported that the SRO program has the potential to be more effective if the officer is well matched with the school. Additionally, the roles and responsibilities of school administration and the SRO need to be clearly defined and supported (Casey, 2007).

Collaboration and networking within these types of partnerships are critical from the early stages and throughout these types of dynamic relationships. Networking among emergency organizations is an important topic in emergency management, but another form of networking and communication involves local government and the citizenry of that community (Ansell \& Gash, 2007). The ability of local, state, and federal government to communicate with members of the community before, during, and after a crisis is of obvious importance to provide necessary safety information (Sadiq, Tharp, \& Graham, 2016). 
Developing, implementing, and the sustaining of school emergency management plans will not be effective without the collaborative efforts of primary stakeholders such as firstresponder agencies, namely, law enforcement (Dorn \& Dorn, 2005; Trump, 2011). A culture of continual improvement in school emergency response must take place through joint-exercises, regular meetings, and continual review of plans. Interagency cooperation and collaboration is a fundamentally imperative process and a major key in effective school emergency management (Ansell \& Gash, 2007; Price, Khubchandani, Payton, \& Thompson, 2015).

\section{Related Literature}

School emergency operation guidelines were developed in collaboration between the U.S. Department of Education, U.S. Department of Health and Human Services, U.S. Department of Homeland Security, U.S. Department of Justice, Federal Bureau of Investigation, and the Federal Emergency Management Agency. It is strongly suggested that public schools utilize these school emergency plan guidelines in preparing school safety emergency initiatives (U.S. Department of Education, 2013). The U.S. Department of Education guide follows the emergency management framework design that closely resembles the comprehensive plan created by FEMA. Currently, 33 states have specific laws that require every school or school district to have a comprehensive school emergency management plan (Council of State Governments Justice Center, 2014).

\section{U. S. Department of Education School Emergency Operations Plans}

The U.S. Department of Education’s “Guide to Developing High-Quality School Emergency Operations Plans" emulates the mission areas of FEMA's disaster response methodology, which is the standard emergency response across the nation (FEMA, 2016). However, the definitions in FEMA's mission areas vary slightly compared to the U.S. 
Department of Education guide that is tailored to meet the needs of the schools. For example, the areas of prevention are actions that may thwart a threatened or actual incident from occurring. Protection focuses on keeping students, staff, and property safe (U.S. Department of Education, 2013). The definitions for mitigation, response, and recovery are similar to the definitions from the FEMA framework.

Kano and Bourque (2008) reported that approximately $56 \%$ of the schools in the United States have plans that follow federal government school emergency management protocols. Additionally, fewer than 20 of the 50 states have an agency whose primary focus is school emergency management (Hull, 2011). Moreover, the majority of schools that have comprehensive school emergency plans rarely conduct coordinated training drills with local first responders (Allen et al., 2010; Kapucu, et al., 2010). The paucity of school emergency planning and lack of coordinated training across the country creates a dilemma that could unnecessarily cost lives and property that could have otherwise been avoided.

In addition to emergency operations plans, schools also need additional security measures on site to complement the prevention and response process. For example, the use of SROs and armed security on campus can serve as a response mechanism and deterrent. Other security measures include security cameras, controlled access to schools, visitor management, and metal detectors that serve as additional tools in security management (Jennings, Khey, Maskaly, \& Donner, 2011). The combination of armed security personnel and security measures provides a comprehensive security element.

The U.S. Department of Education's school emergency plans are provided to schools for use as part of their individual readiness for emergencies. However, other countries that have 
suffered through major calamities have sets of plans that are unique to their country but may be helpful for school emergency planning in the United States.

\section{International Versions of School Emergency Plans}

Countries like Japan and Armenia train teachers to perform significant roles in school emergency management. In Armenia, teachers are trained through an Emergency and Rescue Team concept developed by emergency management trainers. Instruction from these trainers is given to teachers in courses that last from 21- 48 hours depending on teacher grade level.

Teachers study such topics as psychological care, evacuation place management, feeding and caring for students, search and rescue operations, and research and planning in disaster management (Shiwaku, 2014). The training is a comprehensive course for teachers to learn the basics of survival techniques for themselves and for assisting students before, during, and post recovery in a disaster (Shiwaku, Sakurai, \& Shaw, 2016).

In Japan, the Disaster Risk Resilience system requires teachers to not only serve as emergency managers in the schools but also to provide instruction to students about disaster survival techniques as part of the regular school curriculum (Chen, Yu, \& Chen, 2012). Teacher Disaster Risk Resilience training consists of disaster education, identification of disaster prevention, campus disaster response, drill planning, and emergency medical services (Matsuura \& Shaw, 2014). This type of comprehensive training provides an additional level of safety for students, staff, and local community members.

In an effort to become competent in school emergency management, other countries like India, Ghana, Kenya, Haiti, Congo, and Zambia have implemented five-year Disaster Risk Resilience training plans for their schools (Wang, 2016). Disasters are inevitable, but the 
schools in the United States can build resilience in the face of a disaster by practicing and using the techniques taught in Disaster Risk Resilience.

As previously mentioned, the U.S. Department of Education's school emergency plans follow the framework of the FEMA, NIMS, and ICS. For schools that receive federal funding, use of the U.S. Department of Education Safe Schools Guidelines is suggested to receive the benefits provided by the federal government in the event of a crisis (U.S. Department of Education, 2013). These benefits include reimbursement for recovery, which in some cases can cost millions of dollars (FEMA, 2016). Training, support, and important instruction in the use of NIMS and ICS in disaster preparation, response, and recovery are provided by FEMA and the U.S. Department of Education through individualized and free online courses (FEMA, 2016).

\section{Federal Emergency Management Agency}

In 1979, FEMA was created under then President Jimmy Carter by merging several federal emergency management agencies under one organization. In the 1960s and 1970s, several natural disasters brought about increased legislation to assist victims during and after large-scale disasters. After the September 11, 2001 terrorist attacks, Congress passed the Homeland Security Act of 2002, which in turn created the Department of Homeland Security (DHS). Former president George W. Bush placed FEMA under the authority of DHS in 2003 (Perrow, 2011). The DHS and FEMA created the National Incident Management System in 2003 that is a comprehensive national approach to incident management by federal, state, territorial, tribal, and local responders (FEMA, 2016).

Under FEMA, emergency management consists of five predominate missions: prevention, protection, response, mitigation, and recovery. Each category has unique definitions and is a part of the comprehensive framework for emergency management in the United States. 
The individual missions included in the FEMA guidelines are comprehensive yet exclusive to that component of the overall framework. However, each component is equally important in the implementation of emergency protective actions before, during, and after a disaster (FEMA, 2016).

According to FEMA (2016), prevention is the on-going succession of continuous planning, organizing, training, equipping, exercising, and taking of corrective action to ensure effective coordination during incident response. As part of the planning process, vulnerabilities within the area of concern must be evaluated and decisions made to enhance the current resilience in the area (Chung, 2015). For example, a current written plan may be outdated or additional equipment may be needed to provide support. The preparation process therefore is an opportunity to correct shortcomings before an actual event.

Multi-agency training provides the greatest affect and information to make the needed improvements (Kapucu \& Hu, 2014). It is beneficial to provide collaborative training opportunities for first-responder agencies to gain experience in disaster response for certain geographical areas in the United States (Fast, Weaver, Miller, \& Ferrin, 2016). Particular regions in the U.S. present unique challenges such as the West Coast with earthquakes, the Midwest with tornadoes, and the East Coast with hurricanes as examples (Perry \& Lindell, 2003). These unique challenges in various parts of the country make it important to conduct interagency training that is germane to these types of emergencies.

Mitigations for a disaster are the measured steps taken to reduce or eliminate the risk of harm to people or property by taking proactive steps before a disaster (FEMA, 2016). For example, before the Tangshan earthquake that occurred in Japan on July 28, 1976, government officials heeded an early warning system four days before the earthquake by evacuating people 
to safe places and checking vulnerable areas to reinforce certain infrastructures $(\mathrm{Col}, 2007)$. Col added that the preeminent actions taken by government officials potentially saved countless lives.

The response phase includes immediate actions to save lives, protect property, and meet basic human needs (FEMA, 2016). The actions taken by first responders include a variety of factors that will determine the course of action. The term first responders include citizens who are in the immediate vicinity of an emergency who also provide some form of help to those affected. Professional first responders are typically those who work in emergency services like law enforcement agencies, fire departments, and emergency medical services (Quarantelli, 1997). The initial response of emergency personnel will be dictated by the size, intensity, scope, and duration of the disaster impact (Fast et al., 2016; Lindell, 2013; Perry \& Lindell, 2003).

In some weather-related disasters, there is time for people to prepare through evacuation, boarding up windows, or finding a safe place inside of the house. In the event of a sudden earthquake, the preparation time needs to be completed long before the crisis strikes, such as the response to the Paso Robles 6.5 Richter scale earthquake in California on Dec 22, 2003 that caused extensive damage to several buildings and gas lines where over 100,000 residents lost electrical power (McEntire et al., 2012). After the initial quake, the police chief immediately set up an incident command that was later taken over by the emergency services chief until representatives from FEMA arrived. The quick professional response and strong mutual aid agreements made in advance significantly reduced any chaos and assisted the recovery process (McEntire et al., 2012).

FEMA (2016) defined the recovery process of emergency management as the community's return to pre-disaster conditions. A successful recovery process requires a unity of 
effort and expertise of each participating organization while working toward a common recovery objective. The speed and success of recovery can be greatly enhanced by the establishment of certain protocols and plans before a disaster. The recovery process may begin during the response phase of a disaster and will continue until the return to normal daily activities (FEMA, 2016; Lindell, 2013).

\section{National Incident Management System (NIMS)}

Emergency management success is dependent on the involvement of several agencies and levels of government that are part of a global approach to emergency management (FEMA, 2016). Most disasters begin as a local problem, but depending on the speed of onset and size of the disaster, it may quickly become a multi-jurisdictional response with local, county, state, and federal agencies working in a coordinated fashion (Perry \& Lindell, 2003). The creation of NIMS took place in February 2003 in response to organizing a multi-jurisdictional emergency response during a large hazard (Homeland Security Presidential Directive 5, 2015). According to this directive, the purpose is "... to enhance the ability of the United States to manage domestic incidents by establishing a single, comprehensive national incident management system" (p. 1).

According to FEMA (2016), the dominant function of NIMS is to provide a standard set of guidelines for managing disasters, which includes a template for a consistent approach for multiple agencies to perform under hazardous conditions. The NIMS framework provides a plan for responding agencies from various regions of the country to act in a coordinated fashion with limited direction and time wasted in the response. Local, county, state, and federal agencies must attend various training and obtain particular FEMA certifications to remain in federal compliance or potentially lose the opportunity for monetary assistance. 


\section{Incident Command System (ICS)}

As a component of NIMS, the ICS is a management system with six major categories: command, operations, planning, logistics, intelligence and investigations, and finance and administration. The objective of ICS is to allow incident commanders to coordinate and communicate with multiple organizations that are responding to a disaster (FEMA, 2016). As a unity of command, ICS is centralized to allow for a structured environment with plans and procedures in place that are flexible, predictable, and adaptable in any hazard situation (Cooper, 2013). The ICS system can be immediately implemented by any responder that is familiar with ICS such as a police officer, fire fighter, building principal, or emergency manager until they are relieved by a supervisor or experienced incident commander (McEntire et al., 2012).

Quarantelli (1997) and Waugh and Streib (2009) disagreed that the ICS model is the best form of an emergency command system for two primary reasons. It is more centralized, which causes delays in approving and dispatching assistance, and different organizations have unique goals, policies, and interests that can create obstacles with collaboration. As previously mentioned, the U.S Department of Education guide is a working template for schools to utilize in implementing school emergency operations plans.

\section{School Emergency Operations Plans}

School emergency management plans have been a major requirement in our schools for decades (Cray \& Weller, 2011). One of the first recorded school tragedies occurred in the Greenwich Avenue School in New York City in 1851. A fire alarm sounded, and the students tried to escape the fire in mass chaos. The students were not familiar with escape routes, and 40 of them died and several others were injured. After this tragedy, many teachers around the nation began to practice fire drills to train their students to exit the school safely (Golway, 2003). 
Tragedy struck again in New York in 1882, and without the quick reaction of school staff, police, and firefighters, many of the hysterical students could have lost their lives (Heath et al., 2007). Soon after this incident, the New York City Superintendent John Jasper created the concept of "practice fire drills," to teach all students how to exit the building in a safe and orderly fashion. However, the use of fire drills was not commonplace across the nation until around 1961 when fire drills became a part of the safety routine in a school (Taugher, n.d.).

During the 1950s and 1960s, several public and private schools began air raid drills in anticipation of a potential nuclear attack where each student had to hide under their school desk for protection (Heath et al., 2007). Similarly, many school districts located in regions known for certain types of natural disasters practice safety procedures specifically for these types of hazards (FEMA, 2016). Today school principals are required to have fire and other types of safety drills to remain in compliance with county, state, and federal regulations (Trump, 2011).

In recent decades, lockdown drills in schools have become a necessary tool to protect students and staff from an intruder intent on causing harm. School district lockdown procedures vary depending on the type of intrusion. For instance, if there is a credible threat on a school campus, teachers will begin what is termed in some schools a full-lockdown. All doors are locked, lights are turned off, and everyone in the classroom moves away from any windows and doors to wait for an all clear signal. A partial lockdown is used in the event of an attack that occurs at a different location but near the school. The teachers lock all doors and keep students in the classroom but continue instruction until the all clear signal is given or if necessary, upgrade to a full-lockdown (Trump, 2011).

Attacks on school and college campuses are not a new phenomenon. These incidents have been taking place for years. In 1927, one of the worst school attacks occurred in Bath 
County, Michigan that involved an irate school board member who carried out a series of bombings that killed 45 people, and 38 were children (Heath et al., 2007).

Nearly 60 school shootings have taken place in American schools with a rash of armed attacks occurring in the 1990s (Rocque, 2012). One of the worst school shootings occurred in the U.S. at Columbine High School in Littleton, Colorado on April 20, 1999. Twelve students and one teacher were killed by gunfire from students enrolled at the school (Newman \& Fox, 2009). On December 14, 2012, a mass shooting took place on the Sandy Hook Elementary School in Newtown, Connecticut. A single shooter who was the son of one of the teachers entered the campus and shot and killed 20 children and six adults (Agnich, 2014; Leary, Kowalski, Smith, \& Phillips, 2003).

According to several authors, school shootings on America's campuses are rare events that receive intense media coverage (Agnich 2014; Bondu \& Scheithauer, 2014; Dorn \& Dorn, 2005). However, there have been over 200 school shootings since 2013, which is an average of one per week. Relatively few of these shooting events were a national news story, which could mean that school attacks are more severe than some believe (The long shameful list school shootings in America, 2017).

The potential for an armed attack or natural or technical disaster to occur on or near any one of our school campuses in the United States creates an immense need to have an effective school emergency plan. More importantly, these types of plans should be developed in collaboration with first-responder agencies (Gerber \& Robinson, 2009; Hull, 2011; Kano \& Bourque, 2007b). The creation, implementation, and sustaining of school emergency plans requires the expertise and assistance from outside organizations that have a vested interest in the school district (Allen et al., 2010; O'Toole \& Meier, 2011). Local law enforcement, fire 
departments, emergency medical services, county and state emergency managers, counseling offices, and public works should share their expertise in the planning stages. Individual agencies can provide the schools with pertinent information about their agencies including the organization's mission, policies, procedures, and limitations in assisting the school (Sinclair, Doyle, Johnston, \& Paton, 2012). This information will help in understanding each organization's structure and what role it plays before, during, and after a disaster.

\section{SROs}

One of the first known situations of a police officer working in a school district was in 1939 where the Indianapolis School District hired a special investigator who eventually became the supervisor of a group called The Watchmen in 1952. In 1970, the department became the Indianapolis Public School Police. In 1948, the Los Angeles School District created a security group that became the Los Angeles Unified School District Police Department, which now has over 300 officers (Brown, 2006). In Flint, Michigan in the 1950s, schools began using a police officer in the school district as part of their proactive crime prevention approaches (Coon \& Travis, 2012; Cray \& Weller, 2011). A police chief in Miami in the mid-1960s coined the term school resource officer (Brown, 2006; McDaniel, 2001). As of the late 1970s, there were approximately 100 identified school resource officers assigned to public schools throughout the United States (Brady, Balmer, \& Phenix, 2007).

In the 1990s, SRO programs grew out of concern for the surge of violence in American schools (Brown, 2006). However, McDaniel (2001) asserted that the concept of communityoriented policing was the reason for the growth of SROs on school campuses. By the year 2005, the Office of Community Oriented Policing Services supported the use of over 6,500 law enforcement officers assigned to school settings (Kachel, Laslo, \& Nickles, 2005). 
A major push for the use of SROs became a priority following the tragedy at Sandy Hook Elementary School. President Obama's administration passed an executive order to increase the presence of resource officers in schools (Chrusciel et al., 2015). In 2014, the Obama Administration invested over $\$ 61$ million dollars to improve school safety by providing additional resources and research for school safety measures (Wolfe, Chrusciel, Jordek, Hansen, \& Kaninki, 2017).

It is difficult to obtain an exact number of SROs assigned to schools across the nation because of the lack of a standard definition for the position. No matter the particular reason, SROs have become a primary part of school safety practices.

Role of the SRO. School districts and police departments throughout the United States have no clear-cut definition for the SRO or their function, which makes it difficult to integrate goals (Coon \& Travis, 2012). Maranzano (2001) argued that legislators should clarify the vague role of police officers assigned to schools to aid in global understanding. Brown (2006) provided a unique definition for the SROs as “... a new species of law enforcement officer that performs an amalgam of educational, correctional, law enforcement, and role modeling tasks ..." (p. 600).

The SRO is a sworn law enforcement officer employed by a local law enforcement agency that is assigned to a school or schools (Coon \& Travis, 2012; Cray \& Weller, 2011; Trump, 2011). Additionally, the SRO must serve multiple supervisors from their originating police agency and to the assigned school, which creates a natural conflict in this dual capacity (Maranzano, 2001). On one hand, the SRO must serve the school in the capacity of keeping it safe, while on the other, he or she takes on an educational and mentoring role with students.

Duties of the SRO. The duties of SROs vary from school to school but include performing law enforcement functions, counseling students, teaching classes, and being a liaison 
between law enforcement agencies and schools known as the triad model (Brown, 2006;

Lambert \& McGinty, 2001; May, Fessel, \& Means, 2004). Of the SRO duties, teaching classes, providing counseling, and law enforcement and patrol duties take up the majority of the time (Gulen, 2010; Trump, 2011).

McDaniel (2001) clarified the triad difference in explaining that the high school level typically requires a stronger law enforcement presence while the middle and elementary schools require more teaching and counseling from the SRO. In looking at the importance of the triad role of the SRO, Stinson and Watson (2014) discussed the fact that training for the SRO should include mentoring, counseling, teaching, or intervening in a school setting. Cray and Weller (2011) asserted that SROs should receive a minimum of 40 hours of training specifically related to policing in a school setting; however, Stinson and Watson (2014) noted that most states do not require SROs to receive any specialized training prior to taking on the assignment and working with youth.

In taking the training aspect of the SROs a bit further, Lambert and McGinty (2001) purported that generalized SRO classes are not enough because the training should be tailored to meet the needs of the specific school. For example, if the school has a problem with bullying, gangs, drugs, or basic discipline, the training for the SRO should be custom-fitted to take the necessary measures to prevent or stop these types of activities. Brown (2006) noted that law enforcement officers, including SROs, cannot control crime and delinquency in schools because of the multiple variables that exist that influences the crime rate. For example, the SROs cannot change the poverty rate of the community, the number of students in the school, or the crime rate in the surrounding neighborhood. 
The triad model of SRO duties provides a general framework; however, Brown (2006) provided some of the specific daily duties of a SRO, which include but are not limited to traffic control before and after school, assisting with disruptive students, attending parent and faculty meetings, intelligence gathering for local agencies, giving presentations, traveling with school groups on trips, and acting as the liaison between the schools and law enforcement agency. According to the author, the role of the SRO is an amalgamation of functions to keep the school and staff safe.

School administrators, teachers, staff, and student views on SROs. In general, school administration, teachers, and staff are supportive of having SROs on campus because they assert that it makes the school a safer place to work (Coon \& Travis, 2012; May et al., 2004; Trump, 2011). Most school administrators believe that the best security measure in the schools is the SRO because of the training and expertise in law enforcement action and response to emergencies and illegal activities (Chruscial et al., 2015; Myrstol, 2011). However, some argue that there is no empirical evidence that the use of SROs reduces school violence and in some cases have been counterproductive, according to some students (Crawford \& Burns, 2015; Madfis, 2016).

In the context of school emergency management and the collaborative efforts between schools and law enforcement organizations, the likely liaison between the two organizations is the SRO. However, as part of the networking processes, other stakeholders would need to be included in school emergency management such as school administration, teachers, law enforcement supervision, and local political leaders (Burns \& Slovic, 2007; Kapucu \& Garayev, 2013). 


\section{Role of School Administration}

All educational facilities are vulnerable to threats, hazards, or disasters that create major school disruptions (Hull, 2011; Kano \& Bourque, 2007a), and parents, communities, and society have an expectation of the district to care for their children while they are in school (Hull, 2011; Sindhi, 2013). Lindle (2013) argued that parents assume that when their children are in school, they are receiving greater protection than before and after school hours. The author added that the safety and security of children are foregone conclusions, thus any intrusion to the safety of students creates shock. In other words, the safety of students is expected without question, and it is the school district's responsibility to make sure that safety is established. The term in loco parentis is a legal expectation that educators have the best interests of the children in absence of the parents (Hull, 2011).

Building administrators need to realize the importance of school safety management. This enormous task cannot be completed without assistance (Fletcher \& Nicholas, 2015; Trump, 2011; Cray \& Weller, 2011). Similarly, school principals are tasked with raising student test scores, graduation rates, and preparing students to become productive members in society (Glickman, Gordon, \& Ross-Jordan, 2014). A significant element of educational success is implementing a safe learning environment (Cray \& Weller, 2011). A safe school environment, according to Sindhi (2013), can be a direct contribution to an effective learning environment, which enhances the opportunity for students to become productive members of society.

Building principals must also take responsibility for the safety of staff and visitors alike. Sindhi (2013) posited that a safe school environment is the responsibility of school leadership by providing the necessary guidance for teachers and students. According to Fletcher and Nicholas (2015), in many communities, school leaders are looked upon as significant leaders and must 
have the ability to effectively communicate with students, staff, parents, and the community. May, Fessel, and Means (2004) reported that in the case of a disaster, the building principal and teachers are expected to take a significant lead role in dealing with the response activities to prevent or reduce harmful effects.

Both principals and teachers play a dominant role in dealing with the short- and longterm effects on students, parents, and the local community created by a disaster (Roher \& Warner, 2006; Seyle et al., 2013). Additionally, principals play a key role in dealing with the psychological effects on students and teachers from an extreme emergency by preparing and returning the school to some type of normalcy as soon as possible (Fletcher \& Nicholas, 2015). Fletcher and Nicholas also added that principals are key players in the emotional well being of the staff by providing support to allow staff members to deal with their own homes, family, and their students. The ability to effectively navigate the recovery process has a direct effect on how and when school activities can return to normal, quickening the healing process.

As part of the recovery process, principals must ensure that everyone has on-going information regarding community services and updates on a return to school (Watson et al., 2011). Students are especially susceptible to psychological trauma when exposed to a disaster (Fletcher \& Nichols, 2015). Educational leaders must be prepared to offer medical and mental health services for students and staff. Children that receive protection and counseling immediately following a disaster suffer less post-traumatic stress disorder (PTSD) symptoms (Clettenberg, Gentry, Held, \& Mock, 2011). School administration plays a significant role in the post-disaster recovery process by re-establishing the school process and having services available and easily accessible. 
Physical security measures and new legislation are effective tools in preventing potential problems within the schools, but more important is the role that school administrators, teachers, and staff play in keeping the school safe. School building leaders are tasked within each school district, and individual schools staff members play particular roles outside of their typical duties as educators when it comes to school safety. For instance, school administrators typically assist in the development of written school emergency plans or ensure they are updated continually. However, it is equally valuable for school administrators to ensure that interagency drills, tabletop exercises, and multi-agency meetings become part of the overall plan (Allen et al., 2010; Trump, 2011).

Additionally, while there is much discussion regarding preparation, response, mitigation, and recovery, one topic receives little attention yet is crucial in school emergency management readiness - equipment and resources on hand. For example, first aid supplies, food, water, flashlights, and communications, including radios and additional resources for children with special needs, must be located on or near school facilities (Bobo et al., 2003).

\section{School Administration and SRO Partnership}

Building an all-hazards school safety plan seems best addressed by having school administrators and law enforcement personnel work together in developing comprehensive plans, but the relations between these two groups is not always smooth (Coon \& Travis, 2012). Coon and Travis (2012) added that if there is no agreement between schools and police, it is difficult to develop an effective interorganizational partnership. As a part of this dyadic relationship, law enforcement is a potentially important partner in the development and implementation of school emergency plans (Dwyer, Osher, \& Hoffman, 2000). Law enforcement officers can aid in 
bringing an air of authority, crime-fighting expertise, and familiarity in the use of security measures.

Coon and Travis (2012) asserted that there is a moderate amount of disagreement about the amount of time spent on school activities between SROs and school administration. They said, "Police reported greater levels of law enforcement activities such as patrol, response/investigations, and solving school crimes, enforcing truancy laws, and performing sweeps for drugs and weapons" (p. 23). In the area of school safety planning, 95\% of school administrators reported that law enforcement played a role in developing school safety plans, while only $64 \%$ of law enforcement said that they contributed to the development of plans with schools. Thus, there is a gap in the perceptions and the roles played by school administrators and SROs in the development of school emergency plans and readiness. Effective partnerships between schools and law enforcement are a critical factor in creating and sustaining effective school safety plans.

Principals should not only play a role in selecting the SRO for their school, but they would also benefit by gaining an understanding of the role and training of SROs (Lambert \& McGinty, 2002). Both law enforcement and school administration value the importance of school safety, but each has drastically different cultures and backgrounds that could significantly alter this goal (Coon \& Travis, 2012). The authors added that problems are likely to occur when open communication is lacking between SROs and school administrators. Effective school safety is not only the job of police and schools. Leaders in the community, parents, researchers, and local and government officials all play a significant part in school security (O'Toole \& Meier, 2011). 


\section{Role of the Teacher}

Teachers are not traditional emergency first responders; however, oftentimes they will be the first to arrive on the scene of a school disaster and must begin the process of caring for the sick and injured (Kano \& Bourque, 2007a; Kano et al., 2007). Few schools, if any, train teachers to conduct first aid, mental health counseling, sheltering, or search and rescue techniques (Kano et al., 2007). However, teachers and school staff have a duty to protect children (Hull, 2011).

In a large-scale event, emergency assistance may not arrive for hours or possibly days, which means teachers and staff will be tasked to take over first responder duties until assistance arrives (Hull, 2011; Kano \& Bourque, 2007a; Shiwaku et al., 2016). The lack of responder training is not the fault of teachers because their primary duties are educating students with the majority of training time dedicated to learning instructional techniques, curriculum development, and improving test scores (Trump, 2011). School personnel receive little to no emergency management training, and few school districts collaborate with area emergency responders, which creates a chasm in emergency preparation (Allen et al., 2010).

Beyond the idea of acting as an emergency manager in school, the teacher plays a significant part in developing a climate of trust between teachers and students. Students that trust their teachers are more likely to take the risk of reporting bullying and unsafe behavior, which will result in safer schools (Mitchell, Kensler, \& Tschannen-Moran, 2016). Trust among individuals in an organization is linked to collaborative behavior thereby increasing organizational effectiveness and efficiency (Hoy \& Sweetland, 2001). Mitchell et al. (2016) argued that educators cannot change the demographic characteristics of the school, but by developing a level of trust, teachers can contribute to a safer school even with students that come from problematic communities. Boulten et al. (2009) reported that students who do not have a 
sense of belonging and who do not value school as an important part of their life are at-risk for behavior and school related problems.

Teachers play a major role in the relationships with students that can ultimately provide a sense of well-being for students (Sindhi, 2013). School safety has been shown to improve academic success of students in a safe environment and in retaining teachers (Johnson, 2006). Students expect teachers to make them feel safe, and when they observe teachers intervening, the students see them as competent and trustworthy (Mitchell et al., 2016).

School safety is not solely about students; teachers can also be negatively affected in a disruptive and dangerous school environment. There is a direct correlation between teacher burn-out (Brouwers \& Tomic, 2000) and high teacher turnover in the schools that serve high poverty and minority populations, which makes it difficult to close the achievement gap (Jameyson, 2006). Not only is school safety important for obvious reasons, but also the feeling of safety is a direct contribution to student success and teacher retention.

Currently, the majority of schools in the United States do not provide specific emergency management training for school personnel to take on the role of an emergency responder; however, it is in area that should be considered (Kano \& Bourque, 2007a).

\section{Threat Assessment Teams}

Many believe that the deadly shooting that took place on April 16, 2007 on the Virginia Tech campus that took the lives of 32 people could have been thwarted with the use of a threat assessment team (Sampson, 2012). The imminent danger posed by student and shooter Seung Hui Cho may have been revealed by trained stakeholders sharing information. Newman and Fox (2009) reported that Cho had displayed warning signs of mental instability and aggressive behavior with campus police, professors, deans, and employees at the college mental health 
facility. The level of information sharing between these groups is unclear, but this tragedy may have been prevented.

Threat assessment teams are made up of a group of professionals from school staff, school psychologists, mental health counselors, and law enforcement personnel who meet periodically to discuss potential risk factors and warning signs and conduct threat assessments (Trump, 2011). Randozzo and Cameron (2012) described the work of the threat assessment team as an information-gathering process that is analyzed by the group to implement a strategy to help solve the problem if one exists.

Threat assessment teams provide schools with options or alternatives to the zero tolerance suspension policies that were introduced to prevent school violence (Cornell, Gregory, \& Fan, 2011). The authors added that zero tolerance policy mandates a fixed, long-term punishment that is applied in all serious circumstances in the hopes that the removal of disruptive students will improve school climate. In some cases, the zero-tolerance policy does not investigate particular reasons behind the behavior. It simply requires that suspension or expulsion is automatic. For instance, if a student were to bring a weapon to school, suspension or expulsion is automatic; however, an investigation may reveal that the incident showed no malice. The American Psychological Zero Tolerance Task Force (2008) conducted a review, which showed no particular evidence that pointed to the zero tolerance practices improving school safety.

The Commonwealth of Virginia, in response to the Federal Bureau of Investigation and Secret Service recommendation, implemented threat assessment teams in their approach to school safety (Cornell et al., 2011). The end result was the development of the Virginia Student Threat Assessment Guidelines (Virginia Guidelines). According to the Virginia Guidelines, the 
threat assessment teams are made up of a school principal, law enforcement officer, and one or more mental health professionals. The threat assessment protocol begins with a reported threat to a team leader, and a series of interviews are conducted to determine the seriousness of the threat (Cornell et al., 2011). In most cases, the team leader handles the conflict that led to the threat. The authors added that in the most serious cases, the team conducts a comprehensive evaluation, which includes a law enforcement investigation and an evaluation of the student by a mental health professional.

Threats on school campuses can come in a variety of forms. Typically, a student overhears or directly is told of a potential attack on school campus. One of the primary warning signs of an impending attack is a term coined by O'Toole (2000) called leakage. Leakage is a set of clues displayed by a student who intentionally or unintentionally reveals feelings, thoughts, or attitudes in front of other people that may signal the possibility of a violent act. Madfis (2014) reported that several successfully thwarted attacks are due to students that directly or indirectly overheard or saw behaviors from another student and came forward to tell authorities of their concerns. In many cases, it is not easy for students to come forward with negative information about peers.

One way of overcoming the "code of silence" among students is to create a culture of trust between staff and students (Daniels, Royster, Vecchi, \& Pshenishny, 2010; Daniels et al., 2009). Trust is one of the key components of students feeling safe enough to come forward because they feel a sense belonging (Mitchell et al., 2016). Teachers can play a significant role in developing a climate of trust and collaboration with students. 


\section{Security Measures}

Physical security measures seen on campuses across the nation include but are not limited to electronic access cards, locked doors, visitor check-in processes, security camera systems, metal detectors, random searches, zero tolerance policies, and changes in the physical structures of some schools (Gereluk, Donlevy, \& Thompson, 2015). Other types of school security measures include school uniforms, peer mediation, the development of social skills, and adding safety training into the school curriculum (Cherurprakobkit \& Bartsch, 2005).

Most school districts have one or more physical security measures, with the most common being SROs along with a mandatory requirement that visitors check in with the office immediately upon entering the school (Perumean-Chaney \& Sutton, 2012). Crawford and Burns (2015), however, argued that the use of physical security measures, also known as target hardening, has not been proven to reduce crime in schools, and in some cases, create a sense of fear. Chrusciel et al. (2015) disagreed and suggested that law enforcement executives and school administrators believe that the use of SROs and physical security measures is a benefit in keeping schools safer.

The United States Congress enacted several laws to prevent gun violence (Borum, Cornell, Modzeleski, \& Jimerson, 2010). In 1994, the federal government created new legislation to establish the new Gun-Free School Zones Act, which is a part of the Improving America's Schools Act of 1994 (Improving America's Schools Act of 1994, 1995). This act required all schools that receive federal funding to adopt the act by 1995 . Following the Sandy Hook shooting on December 14, 2012, the state of New York created the Secure Ammunition and Forearms Enforcement Act (SAFE) in January 2013 (as cited in Cornell et al., 2011). This act contains a number of firearms provisions such as increased penalties for people who use 
illegal guns and universal background checks for all people who purchase a firearm (NYSAFE Act Gun Reform, n.d.).

\section{Summary}

Physical effects of disasters on schools can be lessened or prevented by hazard mitigation, emergency preparation practices, and heightened awareness coupled with effective interorganizational relationships. Emergency organizations train continuously either as part of a mandatory certification process or an individual and departmental desire to become proficient in their profession. School personnel also attend professional development to enhance their instructional ability to provide excellent educational opportunities for their students. However, educational personnel are also tasked with the responsibility of ensuring their students are safe, but training in this area for teachers is largely ignored because it is not part of the school's primary mission.

A comprehensive review of the current emergency written plans, extent of emergency drills, and exercises, along with a detailed examination of the current interorganizational relationship with local law enforcement, is an important first step toward improving the safety preparedness of school personnel. The written plans must be continually updated with the changing school climate, area demographics, and environmental changes in the area.

Additionally, it is not enough to regularly practice, but the exercises need to be evaluated by objective observers who will provide analysis and feedback (Sinclair et al., 2012).

The events of a natural or technical disaster or an active shooter coming onto campus is rare; however, the absence of emergency planning and preparation is opening the door for loss of life that could have been preventable. School emergency management plans are not only critical to the mission of safe schools, it is also legislated by local, state, and the federal governments 
(U.S. Department of Education, 2013). School administrators that do not heed the potential for a disastrous event to happen at or near their schools are not taking the legal and moral responsibility they undertook when entering into the profession. 


\section{CHAPTER THREE: METHODS}

\section{Overview}

The purpose of this qualitative multiple case study is to explore and provide an understanding of the various approaches to school administrators and law enforcement personnel working together in creating, implementing, and sustaining emergency management readiness in one rural and two suburban pre-k-12 schools located in the Midwest, South, and Southwestern regions in the United States. School administrators and law enforcement personnel must be proactive in designing and implementing school emergency plans that will diminish or prevent harm to students or staff in the event of a hazardous situation or disaster (Kano \& Bourque, 2007). This study could lead to a clear understanding of the interorganizational relationships between schools and law enforcement agencies in developing school emergency plans. This chapter will examine the type of design utilized for this study and why it is the best fit for this topic. The procedures, setting, participants, research design, analysis, and ethical consideration for this study are included.

\section{Design}

For this study, qualitative research is the best method, as it will provide an in-depth analysis in answering the research question from the viewpoint of the participants (Creswell, 2013). The author described the five types of research designs as narrative study, phenomenology, grounded theory, ethnography, and case study with individual and multiple designs. A qualitative multiple case study design was selected for an in-depth analysis of this topic with the use of data collection in the form of interviews, observations, and documents that will include a variety of information from multiple sources. According to Yin (1989), "the multiple case design is often considered more compelling and is therefore regarded as more 
robust" (p. 52). The goal of this qualitative multiple case study was to create an overall understanding of the similarities and differences between the individual cases that result in a prevailing theme (Yin, 2014). In this study, the resulting theme was the interorganizational relationship between two distinct systems with differing mission, goals, and objectives.

Yin (2014) contended that there are five major components of case study design: the questions, its proposition, its unit of analysis, logic linking of the data to the propositions, and criteria for interpreting the findings. Case study methodology allows for an in-depth examination of interorganizational relationships between law enforcement and school administration personnel as it relates to school emergency management.

First, the study's question is key to the entire case study and should be based on the literature, which is narrowed down to a key topic or two that have been previously researched. In this case, the key question pertains to the idea of two organizations with diverse cultures, missions, and objectives collaborating toward a common goal of school emergency management readiness. Second, the proposition directs attention to what is being examined within the scope of the study. The proposition guides the question within the case study that needs to be answered. In other words, how do two distinctive systems, schools, and law enforcement agencies and personnel create an effective partnership? Third, the use of the unit of analysis is used to keep the study focused on the research question and proposition. Selection of the unit of analysis occurs when the primary research questions are accurately specified (Yin, 2014).

The two primary units of analysis in this study were the three separate high schools that employ SROs and their respective law enforcement agencies. Other units of analysis included the individual participant's role within the study, for example, the relationship between the building principal and the SRO and the relationship between the SRO supervisor and the district 
administrator. In this multiple case study, the research question is: How do school and law enforcement personnel with diverse cultures, missions, and objectives collaborate to create, implement, and sustain school emergency management readiness in three high schools from one rural and two suburban pre-k-12 school districts in the Midwest, South, and Southwestern regions in the United States? For this qualitative multiple case study, the units of analysis focused on interagency relationships between the schools and law enforcement agencies in school emergency management readiness.

The fourth component, linking data to the proposition, requires the use of pattern matching, explanation building, and cross-case synthesis, which is described in the data analysis section of this chapter. In a case study analysis, pattern-matching logic compares one observation from the study with a predicted outcome. Explanation building is similar to pattern matching except that the patterns are used to build a description or theme of the case. Cross-case synthesis specifically applies to multiple case studies where each case is independent of the other, but the data from each case is compared (Yin, 2014). This qualitative multiple case study provided a complex collection of information from two independent organizations and personnel that are working in partnership to provide safe schools under their care.

The fifth component, the criteria for interpreting a study's findings, involves the use of rival explanations. According to Yin (2014), “... the challenge is to anticipate and enumerate the important rivals" (p. 34). It is important to therefore to collect data from additional studies that oppose the current study to demonstrate openness to varied conclusions. However, there is a dearth of studies that specifically examines the interorganizational relationships between school and law enforcement in the direct context of school emergency management. For this study, the 
rivals had to include other interorganizational relationships that are similar to each school and the law enforcement dynamic.

This type of research provided themes and patterns that can only be constructed by using multiple forms of data collection and analysis. Qualitative research is required when answering a complex question that contains multiple layers of analysis to the overall theme (Yin, 2014).

\section{Research Questions}

This study sought to answer the following central question and sub questions:

Central Question: How do school and law enforcement personnel in the Midwest, South, and Southwestern regions in the United States with diverse cultures, missions, and objectives collaborate to create, implement, and sustain emergency management readiness?

Sub Question 1: How do personnel from schools and law enforcement create school emergency plans?

Sub Question 2: How do personnel from schools and law enforcement implement school emergency plans?

Sub Question 3: How do personnel from schools and law enforcement sustain school emergency plans?

\section{Setting}

In this qualitative multiple case study, three different school sites along with respective school and law enforcement participants, the assigned SRO, and his or her supervisor were included. Law enforcement agencies that serve the schools and district administrators were also the focus of this study. Yin (2014) recommended the use of three individual cases with a minimum of 12 participants, which establishes a sample size that is large enough to obtain the desired results. 
The cross-case sampling method was used to choose the high schools that provided similar predictive results or contrary results but for predictable reasons as asserted by Yin (1989). To obtain optimal results, this study used one rural and two suburban high schools and three law enforcement agencies that employ the use of SROs. One of the schools is located in a rural setting and the other two are located in a suburban setting within the Midwest, South, and Southwest regions of the United States. Each school has approximately the same number of students.

This study will use the following pseudonyms for the three schools: Region High School \# 1 (RHS \#1), Region High School \# 2 (RHS \#2), and Region High School \# 3 (RHS \#3). RHS \# 1 grades 9-12 has an enrollment of approximately 1,450 students with a student population consisting of approximately 70.5\% Caucasian, $12.8 \%$ Hispanic, 3\%, African American 2.6 \%, Asian 3.4, and $2.1 \%$ of one or more races. RHS \# 2 grades 9-12 has an enrollment of approximately 800 students consisting of a student population that is approximately $68.9 \%$ Caucasian, 3.3\% Hispanic, 23.7\%, African American, 0.9\% Asian, and 2.7\% one or more races. RHS \# 3 grades 9-12 has an enrollment of approximately 1,750 with a student population consisting of approximately 35.9\% Caucasian, 54.7\% Hispanic, 2.7\% African American, 4.6\% Asian, and $1.6 \%$ one or more races.

The law enforcement agency that has jurisdiction over RHS \#1 is known as the Region \# 1 Police Department, which employs 135 certified full-time police officers with one full-time officer assigned to the high school as a full-time SRO at RHS \#1. The law enforcement agency that has jurisdiction over RHS \#2 is known as the Region \# 2 police department, which employs 40 certified full-time police officers with one officer assigned to RHS \#2 as a full-time SRO. The law enforcement agency that has jurisdiction over RHS \#3 is known as the Region \# 3 
Police Department, which employs 55 certified full-time police officers with one officer assigned to RHS \#3 as a full-time SRO.

\section{Participants}

This qualitative multiple case study used participants from three high schools and three law enforcement agencies to create certainty with the results as suggested by Yin (2014). Creswell (2013) asserted that the use of purposeful sampling is best suited to gain an understanding of the central phenomenon. The selection of participants from the schools and respective law enforcement agencies in this study was done for this purpose. The selection of the participants provided literal replications in this embedded design where each case was a whole study by itself yet was able to provide convergent evidence. It was assumed that the selection of participants from each site provided enough information to gain similar or contrary results as noted by Yin (2014).

Participants interviewed from each school included the high school principal or designee and a representative from the district office. This meant the superintendent, assistant superintendent, or the head of safety and security, if there was one available, was interviewed as a participant. The personnel interviewed from the law enforcement agencies included the SRO assigned to the high school and his or her immediate supervisor. There were 12 participants between school administration and the respective law enforcement agencies covering each school. Verbal permission to conduct this study was provided by each of the school districts and law enforcement agencies.

\section{Procedures}

After receiving approval from the dissertation prospectus committee, this study was forwarded to the IRB for approval. Once IRB approval was received, consent was obtained from 
the district superintendents of the respective school districts and the top administrator from the respective law enforcement agencies. Upon receiving permission from the district superintendents and law enforcement command staff, this researcher contacted each participant including the high school building principal, district leader, assigned SRO, and the officer's immediate supervisor to explain the purpose of this study and to obtain consent for their participation.

Once consent was obtained, this researcher scheduled interview dates and times at the convenience of the participants. All interviews were recorded using two recording devices. A professional transcriptionist completed the transcriptions of the interviews. The documents were copied and kept in a locked room and password-secured computer to protect the anonymity of the participants and locations. Pseudonyms were used for the interviewees, the names of the school districts, high schools, and law enforcement agencies.

\section{The Researcher's Role}

As the "human instrument" in this study, it was crucial for me to understand the theoretical issue of the case study because interpretations of the data are needed to determine if there are contradictions suggested by Yin (1989). I am a director of crisis intervention and safety at a school district with over five years experience in school administration and over five years experience as a high school teacher. Before a career in education, I was a member of law enforcement with 20 years experience that included roles of investigator, SRO, gang unit, lieutenant, and chief of police. My background in law enforcement and education provides a unique perspective and understanding of the role of a school administrator and that of a first responder in school emergency planning efforts. 
The research for this qualitative multiple case study did not take place in my school district to avoid any bias or conflict of interest. I do not have connections with any of the participants, school districts, or law enforcement agencies other than through casual contact as a school administrator and law enforcement officer in the area before beginning this study.

\section{Data Collection}

Yin (2014) said, "Six sources of evidence can be the focus of data collection for case studies: documentation, archival records, interviews, direct observations, participant-observation, and physical artifacts" (p. 85). A triangulation method was used for this study to include site documentation, interviews, and artifacts. According to the author, the convergence of multiple forms of data is in line with the descriptive case study design. For this case study, the multiple forms of data included:

- Copies of school security plans, SRO job descriptions, safety training and exercise documentation, and minutes from school emergency committee meetings and MOUs

- Copies of the DOE Guide for Developing High School Emergency Operation Plans, the AWR-148 Guide: Crisis Management for School Based Incidents

- Copies of detailed notes from interviews and observations, photographs, videos, or maps that are pertinent to this study

As part of the data collection process, all interviews will be recorded and transcribed for analysis. Yin (2014) stated, “... the most important advantage presented by using multiple sources of evidence is the development of converging lines of inquiry, a process of triangulation and corroboration" (p. 115). This multiple case study presents a conclusion that is more highly trustworthy than a single case study because of the use of multiple sources of evidence, artifacts, and individual case studies. 
The data collection process commenced with document collection and analysis for developing theoretical ideas for use during the interview process. The data obtained from the interviews was used to answer the research question.

\section{Interviews}

Interviews will be primary source of data for this study. According to Creswell (2013), semi-structured interviews are one of the preferred methods of data collection for a multiple case study because of the open-ended type of questioning. In a case study, the interview is a guided conversation more than a series of structured questions (Yin, 2014). The conversational style of questioning allows for an easy flow rather than a dogmatic inquiry. Each of the interview questions is to be “... open-ended, general, and focused on understanding your central phenomenon in the study" (Creswell, 2013, p. 163). While interviews are a primary source of information, corroboration of the interviews with other sources of evidence is of value as well (Yin, 2014).

Interviews were completed at a prearranged date and time at the convenience of the interviewee. Each interview was conducted at the work place of the participant in a place where no one could overhear the interview. For data collection purposes, the interviews were recorded to maintain a record of the interviews and so that they could be transcribed. As previously mentioned, for the protection of the participants, school districts, and law enforcement agencies, pseudonyms were used. Table 1 shows the questions that were used for this study. 


\section{Table 1}

\section{Interview Questions}

1. Please introduce yourself, length of time in your current position, and length of time in your career.

2. Describe your working relationship with others (e.g., SRO, SRO supervisor, principal, district level participant)?

3. Discuss the process used to create the current written school emergency plan.

4. Describe the process that was used to implement the school emergency plan.

5. Discuss the training methods used to sustain school emergency management readiness.

6. Describe the barriers that affect the interagency relationship with others (e.g., SRO, SRO supervisor, principal, district level participant) in the context of school emergency management

7. What influences affect the interagency relationship with others (e.g., SRO, SRO supervisor, principal, district level participant) in the context of school emergency management

8. How do the mission objectives for your school affect the interagency relationship with school and law enforcement personnel in school emergency management?

9. What do you believe are the strengths of your working relationship with others (e.g., SRO, SRO supervisor, principal, district level participant) in the context of school emergency management?

10. What do you believe are the weaknesses of your working relationship with others (e.g., SRO, SRO supervisor, principal, district level participant) in the context of school emergency management?

11. How do you foster the relationship with others (e.g., SRO, SRO supervisor, principal, district level participant) in the context of school emergency management

12. Describe the process used to come to a consensus with others (e.g., SRO, SRO supervisor, principal, district level participant) in the context of school emergency management.

13. Describe the process used to examine and assess written plans, training, and the ability to effectively respond to an emergency event involving the school.

14. What steps can be taken to improve the interagency relationship between school and law enforcement personnel?

Interview question one was used to obtain background and demographic information

from the participants and describe their position within the framework of this study. Each of the participants will provide unique perspectives to the overall research question of the interagency relationships in school emergency management. 
Interview question two provided insight into the actual perception of the relationship between the schools and the law enforcement agencies (Lambert \& McGinty, 2002; SchnobrichDavis \& Terrill, 2010; Cray \& Weller, 2011). This information was crucial to the entire framework of this study and the research question. According to Yin (2014), this is a Level 2 question that caters to a unit analysis in the case study, which is the individual perception about the interorganizational relationship between schools and law enforcement.

Interview questions three and four were opportunities to explain the process that was used in the development, implementation, and sustaining of the school emergency plan (Hull, 2011; Kano \& Bourque, 2007b; Roher \& Warner, 2006; Trump, 2011). These questions garnered information about the sources, criteria, and thought process of how and why the current emergency plan was selected.

Interview question five was a fact-finding question to determine types of training, exercises, drills, meetings, and certifications that are used in sustaining the school emergency plans. School emergency management requires written plans, but of equal importance is the need to train, educate, and development a culture of improvement (Fast et al., 2016; Perry \& Lindell, 2003; Roher \& Warner, 2006).

Interview questions 6 and 7 were related to interorganizational relationships and some of the obstacles and influences involved in bringing two individual agencies to work together effectively (Coon \& Travis, 2012; Jackson, 2002; Schnobrich-Davis \& Terrill, 2010).

Interview question eight was similar but different than question five in that it questioned the primary mission, goals, and objectives of each organization and how they affect the interorganizational relationship (Ansell \& Gash, 2007; Coon \& Travis, 2012; Kapucu \& Hu, 2014). It was important to identify the differences and similarities in the organizations but also 
to analyze the process that is used to collaborate between agencies (Kapucu et al., 2010; Perry \& Lindell, 2003; Quarentelli, 1997).

Interview questions nine and 10 were questions directly related to the strengths and weaknesses of the relationships between the building principal and members of the law enforcement agency (Gulen, 2010; Trump, 2011; Cray \& Weller, 2011; Wolfe et al., 2017).

Interview question 11 was directed at fostering relationships between individuals and the agencies (Wolfe et al., 2017). Regular meetings and training sessions are significantly important in developing effective relationships; however, opportunities to meet outside of the business environment allows for an additional layer of relationship and trust building (Kapucu et al., 2013).

Interview question 12 asked participants for a description of the process that both organizations use to come to a consensus in the joint decision-making process (Ansell \& Gash, 2007; Kapucu et al., 2013; Kapucu \& Hu, 2014). It was important to learn how decisions are made when both organizations have a stake in the outcome. Additionally, this question assisted in identifying the leaders from each organization and their role in the decision-making process.

Interview question 13 provided information on the fact-finding process that determines the effectiveness of school emergency plans. According to Sinclair et al. (2012), it is crucial not only to prepare for disasters through training and exercises but also to have an outside unbiased auditor to review the written plans and exercises to provide an analysis of the current state of readiness.

Interview question 14 provided insight into the opinion of each participant to improve the interorganizational relationship between school and law enforcement personnel in developing, implementing, and sustaining school emergency management plans. 


\section{Documents}

The following artifacts were gathered and analyzed as additional data for this study:

- Interview transcripts

- Copies of school security plans

- Detailed notes from interviews and observations

- The Guide for Developing School Emergency Operation Plans

- The AWR-148 Guide: "Crisis Management for School Based Incidents"

Additionally, copies of safety training and exercise documentation, minutes from school emergency committee meetings, MOU, and the school resource officer job description were utilized. All artifacts that are gathered were kept in a secure location. As this study progressed, some additional artifacts were included or removed if they did not provide information conducive to it.

\section{Artifacts}

One of the tentative goals in this case study was to gather documents related to school emergency planning, safety drills, minutes from safety meetings, safety audits, and any other artifacts related to school emergency management readiness. Creswell (2013) asserted that documents, audits, photographs and other forms of artifacts provide a unique insight into the types of documents that schools use in emergency management readiness; however, bias may occur due to participants' manipulation of the documentation. The artifacts allowed for an opportunity to see some types of documents preserved by the schools for record-keeping purposes. The researcher's role was as a non-participant to examine the various documents for further analysis of each school's record keeping of school emergency related events. 


\section{Data Analysis}

Analysis of data will include a variety of techniques and strategies to gain a thorough understanding of each case within this study. First, the use of pattern matching was used to determine if there were patterns that emerged which were relevant to this study. For example, "in each outcome, the initially predicted values have been found, and at the same time alternative patterns of predictive values emerge, strong causal inferences can be made" (Yin, 2014, p. 137). The use of pattern matching assisted in finding relevant and compelling themes that provided an explanation for the differences or similarities in findings.

Next, a coding system was used to put interview information and particular events in numerical forms, or in different arrays. This process assisted in creating data displays to provide an organized method of analyzing the data (Yin, 1989). Yin (2014) discussed the use of theoretical propositions as the cornerstone of this type of data inquiry. For this study, the theoretical framework of interorganizational relationships provided an analysis for the relationship between school administration and law enforcement personnel.

In multiple case studies, the use of cross-case analysis and synthesis is important in providing a method to examine each case as a separate study. This technique further provided opportunities to analyze the information from each of the cases and units of analysis that were studied. Lastly, the use of explanation building was used to provide an explanation for certain phenomenon as they appear with a set of causal links of potentially theoretical significance about how or why something occurred (Yin, 2014).

The data was uploaded into the ATLAS.ti software program that stores and organizes data for further analysis to identify common passages, create visual pictures of codes, make comparisons, and create a working template for coding purposes. As part of the disaggregation, 
a multiple bounded case study analysis of each school district and law enforcement agency provided various sources of documentation that were used in triangulating the data to obtain a visualization of converging lines of data (Creswell, 2013; Yin, 2014).

\section{Trustworthiness}

For this study, trustworthiness was built on a four-step foundation using the following techniques. First, construct validity was developed through an operational set of measures and subjective judgments using multiple sources of evidence. A chain of evidence was then established, and the use of key informants assisted in analyzing and auditing the results. Second, internal validity was used to provide an explanation as to how and why events happened through the use of pattern matching, explanation building, addressing rival explanations, and using a logic model. Third, external validity was determined if the findings from the study were generalizable beyond this study through replication logic. Replication logic is a way of testing the theory with all three case studies (Yin, 2014).

\section{Credibility}

One of the primary steps in determining the credibility of this study was the use of member checking. Creswell (2013) described member checking as the data analyses, interpretations, and conclusions taken back to the participants to have them verify accuracy.

\section{Dependability and Confirmability}

Achieving dependability and confirmability required auditing of the entire research process and the steps that were taken to obtain all of the data used in this study. Dependability in this case meant that the results were subject to change and instability (Creswell, 2013). The researcher had to adjust to new or unexpected information and share the results accordingly. Creswell also explained that some of the validation steps in a case study involve a peer review 
clarifying researcher bias and an examination by an external unbiased auditor to review the process and an assessment for accuracy. Such was followed in this study.

\section{Transferability}

Transferability of a case study means another researcher will be able to replicate the study in a different or similar setting. The use of thick, rich description of the data collected and the analysis, setting, and participants provided the information required for another researcher to conduct the same study (Creswell, 2013). Another analytic technique to ensure transferability is pattern matching. Yin (2014) explained that pattern matching is the examination of the theoretical proposition used in the analysis. If a study is replicated but uses a different theoretical framework, the final analysis will differ even if the same study methodology is used in both studies.

\section{Ethical Considerations}

The IRB and all committee members approved this study before the collection of data. Consent forms were obtained from each participant with pseudonyms used to protect individual identities and locations. All data was kept in a locked room or on a password-protected computer and backed up using thumb drives, which also were stored in a locked cabinet. The recorded interviews of the participants and the recorder were stored in a safe, locked location.

One of the ethical considerations in this study was to remain unbiased. In my attempt to remove any personal bias, bracketing was used to allow for a fresh lens in which to conduct a multiple case study (Creswell, 2013). Additionally, I had an outside auditor analyze the findings to ensure that researcher bias was not reflected in this study. 


\section{Summary}

For this study, a qualitative multiple case study methodology was used. This study added to existing research in the area of school and law enforcement relationships in creating, implementing, and sustaining school emergency management readiness. The justification for the design and reasons for choosing participants were presented in this chapter.

The selection of participants, data collection, and all other pertinent information followed IRB guidelines. This researcher used various methods of data collection and analyses to obtain the most accurate results for this study. Protection of participants was a major priority throughout this process. All documents and materials obtained during this study were kept in a secure location that was either locked or password-protected with backup devices that were also protected. 


\section{CHAPTER FOUR: FINDINGS}

\section{Overview}

This multiple case study seeks to describe the interorganizational relationship between school and law enforcement personnel in creating, implementing, and sustaining school emergency management readiness located in the Midwest, South, and Southwestern regions in the United States. This study used various data collection methods including participant interviews, documents, artifacts, and member checking to search for codes, themes, patterns, and ideas to describe the interorganizational relationships between school and law enforcement personnel.

A central research question and three sub-questions explored the interorganizational relationship between school and law enforcement personnel in the creation, implementation, and sustaining of school emergency management readiness. The first sub question examined the process of how school and law enforcement personnel create school emergency plans for each site. The second sub question explored the process of how school and law enforcement personnel have implemented school emergency management plans at each site. The third sub question focused on the processes of how school and law enforcement personnel sustain school emergency management plans at each site.

\section{Participants}

This qualitative multiple case study included four participants from each high school for a total of 12 participants who agreed to complete a face-to-face interview as one part of the data collection process. Each participant shared his or her individual experiences and role in the school emergency planning, implementation, and sustaining process. To protect the identity of the participants and their unique roles within their school sites, each participant was assigned a 
name that begins with a letter that correlates with their role as a participant. For example, Daniel, Dwight, and Darryl are all district administrators. Pamela, Patricia, and Peggy are all principals. Samantha, Sally, and Sharon are all SRO supervisors, and finally, Ronald, Rodney, and Roger are all SROs in this study. Actual participants included three male district administrators, two male and one female principal, two male and one female SRO supervisor, and two male and one female SRO. All of the participants had less than five years in their current roles at the time of the interviews; however, most of the participants had between five and 20 years in their respective careers as shown in Table 2. 
Table 2

Participant Demographics

\begin{tabular}{llcc}
\hline Name & Position & Overall experience & \multicolumn{2}{c}{$\begin{array}{c}\text { Experience at current } \\
\text { school }\end{array}$} \\
\hline Daniel & District Administrator & $20+$ & $0-5$ \\
Dwight & District Administrator & $15-20$ & $0-5$ \\
Darryl & District Administrator & $20+$ & $6-10$ \\
Pamela & Principal & & \\
Patricia & Principal & $20+$ & $6-10$ \\
Peggy & Principal & $10-15$ & $0-5$ \\
Samantha & SRO Supervisor & $20+$ & $0-5$ \\
Sally & SRO Supervisor & $10-15$ & $0-5$ \\
Sharon & SRO Supervisor & $10-15$ & $0-5$ \\
Ronald & SRO & $20+$ & $0-5$ \\
Rodney & SRO & $5-10$ & $0-5$ \\
Roger & & $15-20$ & $0-15$ \\
\hline
\end{tabular}

\section{Daniel}

Daniel has spent his entire career in education, first as a classroom teacher, then as an assistant principal, and later as a building principal until he moved into his current role in district administration. During his tenure as a building principal, he worked in different districts, each of which has had an SRO assigned in his building. Daniel's experience working with an SRO as a building principal and currently as a district administrator has provided different results. In two 
cases, his previous relationship with the SRO was distant due to lack of time working together on a daily basis; however, while in another high school and acting as a building principal, Daniel worked closely with an SRO daily to keep an open line of communication although the agency that employed the SRO did not show as much support, thus creating a weak relationship between himself and the SRO.

In his current role as a district administrator, Daniel reported a strong sense of camaraderie with the SROs and the SRO supervisor because of support from the police agency and the desire to promote school safety within his school district. He described the relationship as, "building that positive relationship and understanding each other's roles and understanding that we are not here for each other, we're here to keep the kids safe." Daniel has a unique understanding of the relationship between schools and law enforcement because of his experiences.

\section{Dwight}

Dwight has worked in education for over 15 years and has more than three years' experience in his current role as an assistant principal and district administrator over school safety. In this capacity, he works closely with the SROs throughout the district and leads monthly group safety meetings. Dwight has played a significant role in developing the current district safety plans, emergency drills, and training in the area of school safety.

Over the past three years, Dwight has been developing a new school safety plan together with district employees, including building principals and school safety liaisons. As part of the new school safety plan, the district added safety liaisons on each campus. The liaisons are school staff members who have the additional assignment of being a member of the district safety committee. Additionally, he has attended yearly state conferences for SROs to further his 
understanding of the role of the SRO, which has helped promote his relationship with the SROs. Dwight reported that his position is that school administrators should attend SRO training with their counterparts but SROs should in turn attend school administration conferences.

\section{Darryl}

Darryl has worked in education for over 20 years and has been in his current position as a district administrator for over five years. He plays a significant role in developing training programs and administering regular school safety meetings with the SROs and building administrators in the district. According to Darryl, one of the district goals is to have "concept of uniformity" across the district.

In his role, Darryl works closely with the SROs and building principals. His goal is to continue to bolster the school safety concepts and working relationship with the area police departments. One important step in developing uniformity has been to create an MOU that is specifically tailored to the relationship and objectives of the school and local law enforcement. Darryl has been instrumental in creating the district's current school emergency management plans, which have been in development for several years.

\section{Pamela}

Pamela has worked in education for over 20 years serving as a teacher and previously as a building administrator and district administrator. Her current role is as a building administrator (principal). In the area of school safety, Pamela shares her role in the building with her administrative staff. The staff plays a more significant role in the day-to-day school safety operations. Although Pamela reported being "well versed" in the area of school safety, she delegates the majority of safety practices to her assistant principals. Overall, her opinion is that school safety within her district is a priority because of the time dedicated to conducting drills, 
training school staff and on-going time spent on auditing their school emergency management plans. Nonetheless, Pamela said, "It's really tough to get professional development time for safety because we are focused on learning." She acknowledged the importance of school safety, but realizes that there is a limited amount of time during the school year to spend on school safety training.

\section{Patricia}

Patricia has worked in education for over 20 years as a teacher, assistant principal, and building principal. In her current role as a building principal, she works closely with the SRO to develop plans and strategies to enhance the school safety program. This year Patricia decided to include the SRO in all administrative meetings and to make the SRO a part of the administrative team. The practice of having the SRO included in the administrative meetings is relatively new, but in her opinion, there are positive results from this decision. Patricia shared, "I notice a difference with cooperation and buy in [with the SRO]." However, the SRO is assigned to other schools within the district and is not available all of the time, which creates a gap in SRO services at her school. Patricia is well versed on school safety concepts but added that her district administrator is tasked with the responsibility of creating and sustaining their school emergency management plans.

\section{Peggy}

Peggy has worked in education for over 20 years as a teacher, assistant principal, and building principal. She is relatively new to the district in her current position, so she has not played a role in the development of the school's safety planning. During her career, Peggy has worked with SROs in other districts as a building principal. She revealed that the personalities of the SROs have played a distinct role in their working relationship. 
In Peggy's current capacity, assistant principals and deans handle the day-to-day safety operations while working closely with the SRO. Her goal is to find time to work closely with the SRO on a regular basis, although her duties have not allowed much time to develop this relationship. Peggy said, "the more aligned [a person is] terms of objectives, the better the relationship is going to be and the better the communication [will be]." Before arriving at this high school, the previous administration made the SRO a member of the administrative staff and one who attends all school administrative meetings. She welcomes the process of having the SRO as a part of the administrative team.

\section{Samantha}

Samantha has over 15 years experience in law enforcement, although she has never served directly as an SRO. She has a dual role working in investigations and acting as a supervisor of the SRO program. However, since the SRO's work with school administration on a daily basis, Samantha reported that her role is to be a mentor or an advisor when needed.

Samantha works with district administration throughout the year to discuss upcoming training plans and the current state of school safety throughout the district. She said, "Finding those windows of opportunity to do the drills can be very challenging." Samantha asserted that the SRO program in her police department is one of the strongest in the nation due to positive comments that she has received at national SRO training seminars.

\section{Sally}

Sally has over 20 years experience in law enforcement and currently works as an SRO and SRO supervisor. She handles SRO responsibilities at her assigned schools while also serving in the role of a supervisor. Sally described her role as "being the person that makes sure it is carried out." She works closely with school administration across the district on a daily 
basis. Sally added that she also handles supervisory duties over patrol in her agency when needed. Her multiple roles within the police department makes it difficult at times to focus on all of the schools; however, when the opportunity arises, she enjoys working with the students oneon-one in a mentoring capacity.

\section{Sharon}

Sharon is currently assigned to the investigations unit as a supervisor, but she also manages the SRO program. She asserted that the SRO assigned to the school works well with school administration. Sharon only steps in when necessary or when the SRO or school administration asks her to do so.

According to Sharon, "The SRO is a good fit with the day-to-day operations of working with administrators." Additionally, she enjoys a solid working relationship with school administration and makes herself available if needed. While the SRO reports to Sharon on a daily basis, it is only to provide updates or discuss incidents that may have occurred that she needs to know about. There are times, however, that she assists with school emergency drills as an observer or consultant.

\section{Ronald}

Ronald has been in the law enforcement field for approximately five years although he is relatively new to the SRO role. He is assigned solely to the high school but assists at other campuses as needed. Ronald reported about the importance of his current role and ensuring the safety of students and staff. He makes it a point to remain visible throughout the building while also serving in a multitude of capacities. For example, Ronald may handle some counseling services with students, teach classes on safety, serve in traffic control, conduct criminal investigations related to school matters, and work with school administration on law enforcement 
matters to name a few duties. He said, "I don't have enough time to accomplish all of the law enforcement objectives, [and] all of the safety objectives." Ronald added that he works closely with the assistant principals and school nurse to assist in working through student related issues.

\section{Rodney}

Rodney has worked in law enforcement for over 15 years and is currently assigned as an $\mathrm{SRO}$, a role he has had for over five years. He is assigned to the high school but has responsibilities at other campuses as well. His relationship with school administration has been positive because of the administration's willingness to communicate at all hours to assist each other. Rodney described his relationship with administration by saying, "At any time, we speak on and off work hours when needed."

Rodney shared that more training is necessary throughout the district to enhance current school safety readiness. He said, "That's why I preach, train, train, train on both sides, education and law enforcement." Rodney also reported that he wishes he had more time to handle all of the emergency drills and calls for service to his assigned schools.

\section{Roger}

Roger has worked in law enforcement for over five years and has been in his current position for three years. He is solely assigned to his campus full-time as the SRO and is a part of the administrative team. Roger regularly attends administrative meetings and is used as the law enforcement liaison. He expressed his role "as not just an SRO, [but] a police counselor."

Roger reported having an excellent working relationship with school administration, but he primarily works with the deans or assistant principals in day-to-day school safety matters. As part of the administrative team, he also assists with the Restorative Justice Program, truancy, and 
drug and alcohol issues with students. As the SRO, Roger plays a significant role in the planning of drills and other safety events.

\section{School Sites}

\section{RHS \#1}

The school safety program in this district was built upon a school safety plan template provided by the state department of education. RHS \# 1 has used the template but has also tailored revisions that are specific to each school building. Each building principal ensures that each school plan is current through an auditing process and conducting regularly-scheduled emergency drills.

RHS \# 1 utilizes a regular schedule to conduct emergency drills, which are reported to the state department of education. School administration, the SRO, and SRO supervisor assess each drill, exercise, or live event after it concludes. The assessment includes specific information regarding actions of teachers and staff members' reactions to and handling of the event, whether they are positive or negative. As a continual improvement process, the results of each assessment are also shared with faculty and staff.

This school district also participates in a yearly, countywide safety summit attended by school administration, law enforcement, and fire personnel. There they share information related to school safety. Several topics discussed in this daylong event include an active shooter on school campuses, "Run, Hide, Fight," and school law updates.

The law enforcement agency for RHS \#1 offers additional school safety training for school staff members on Saturdays for those interested in attending. Furthermore, the district is working to develop a video emergency training series in addition to current training offered in the area of sexual harassment, health and wellness, and child abuse reporting. Due to time 
constraints of faculty, offering classes online could provide more opportunities to stay current on school safety concepts.

\section{RHS \#2}

The current implementation of the school safety program began in earnest approximately three years ago with the collaborative efforts of district and law enforcement administration. The district safety program utilizes a template developed by the state, but each school site also has plans that are tailored to meet the needs of their school. As part of the school safety planning, specific mandatory drills are conducted on a monthly basis, which include tornado, fire, and lockdown drills. Upon the completion of each event, school administrators and the SROs complete an after-action report to ensure that any discrepancies are addressed and to improve any issues before the next drill. After the debriefing of emergency drills or events, the principal discusses the after-action report during regularly scheduled faculty meetings.

At RHS \# 2, the SRO plays a major role in planning, conducting, and evaluating each of the emergency drills for each school building. Given the small number of SROs assigned to the district, the SRO at this high school is also responsible to handle safety-related responsibilities at other school locations, which periodically creates a gap in service. The district employs the use of an emergency management software program that allows first-responder personnel and school staff members to receive emergency notifications immediately after an emergency alert has been sent. The information from the alert includes the location, type of emergency, maps of the buildings, and real-time information via an application on cell phones.

\section{RHS \#3}

The development of the current school emergency management plans for this school district began approximately 15 years ago when a nearby city fire department received a federal 
grant. This school district and other first-responder agencies then began collaborating on best practices to use in the event of an emergency. While the state also plays a role in school safety planning, this school district employs plans that closely mirror concepts from the DHS, FEMA, NIMS, and ICS. School safety plans are consistent with an all-hazards approach. As part of the overall plan, school administration must complete EMI training provided online through FEMA. District administration conducts on-going safety training, which includes tabletop exercises and safety related discussions with building principals during regularly scheduled administrative meetings. Additionally, the district administrator meets with the SROs on a monthly basis to ensure that there is uniformity in operating philosophies from a juvenile justice standpoint and general policing concepts at school sites.

Mandatory drills assigned by the state are also completed on a monthly basis, which include fire, lockdown, and evacuation drills. Each of the building principals is responsible for ensuring that each drill is conducted; however, the assistant principals and deans have the primary duty of handling safety protocols along with the SRO.

As part of the administrative team, the SRO attends all school administrative meetings and is considered an equal in handling disciplinary and safety matters involving students and staff. Decisions made concerning student discipline are handled collaboratively with the assistant principals and deans on campus.

Creating school emergency management plans for school districts is a priority among school districts throughout the United States. The federal government does not make it mandatory for every school district to have an SEP in place, although it is highly recommended. To take it a step further, the federal government provides free training, safety plan templates, and other tools for schools to use in implementing their emergency plans. The primary 
implementation of school emergency management plans was dictated by the state educational agency for each of the school sites.

In this qualitative multiple case study, each of the three high schools created, implemented and have sustained school emergency management plans to some degree. Each school has unique and similar processes that pertain to their respective school emergency management readiness. The participating school sites were located in the Midwest, South, and Southwestern regions of the United States.

\section{Results}

The results for this qualitative multiple case study are reported in detail using a systematic approach that assists in clarifying the subject matter. The research process for this study included an analysis of relevant documents, in-depth interviews, and triangulation of data to facilitate validation of the data collected from multiple sources. The findings are presented by a cross-case and within-case synthesis. This is followed by a discussion of various codes that were identified and narrowed into major themes during the data analysis. The themes that emerged after a thorough analysis of the data are then presented. Lastly, an examination of participant answers to the central question and three sub research questions are presented.

\section{Cross-Case Synthesis}

The cross-case synthesis sought to illustrate the process used to plan, implement, and sustain school emergency management plans across the three high schools included in this study. This type of cross-case synthesis examined the interorganizational relationships between school and law enforcement personnel in the creation, implementation, and sustaining of school emergency management plans. The roles of each participant across cases are further analyzed to seek similarities and differences within the various roles. A cross-case analysis examines some 
of the barriers that affect the interorganizational relationships between school and law enforcement personnel.

\section{Cross-Case Analysis}

The cross-case analysis compared each of the individual roles across each of the sites, RHS \#1, RHS \#2, and RHS \# 3. A cross examination analysis was used to juxtapose the similarities and differences between the unique roles of their positions within the district. In this multiple case study, there were three district administrators, three principals, three SRO supervisors, and three SROs. Each of the district administrators, principals, SRO supervisors and SROs were compared and contrasted as part of the analysis process.

District administrators from each district play a substantial role in the creation of their current school emergency management plans. In each of these cases, the district administrators are responsible for ensuring that their school emergency management plans adhere to state education agency standards. For example, when Daniel began his current position, the state department of education departments changed the safety plan template. He worked closely with SRO supervisors, SROs, and principals to enact the change. David also observed that the current district safety plans were not up to date, so he decided to create new plans using the state template. However, Darryl did not necessarily use the state template but worked closely with a local fire department and developed an all-hazards plan that closely resembles the FEMA and ICS systems for emergency management.

The district administrators in each of these cases are responsible for ensuring that their school emergency management plans adhere to state education agency standards. Additionally, district administrators must oversee and ensure that the building principals carry out the mandatory emergency drills according to the schedule. 
Principals in this study played varied roles in the implementation and sustaining of school emergency management plans, which require taking written plans and putting them into practice using regular training and drills. In two of the schools, the assistant principals and/or deans have taken a more active role in ensuring that emergency drills are conducted throughout the school year. This observation is not to suggest that principals do not take safety seriously; however, delegating this responsibility makes sense to provide the building principal more time to focus on educational priorities. In RHS \# 2, the building principal has been the one to take lead for their building under the guidance of the district administrator.

Supervisors of the SROs in two of the districts are located at off-school sites and are assigned to the investigative division; however, they have the added responsibility of supervision of officers within their command. In RHS \# 1, Samantha works directly with district administration but also makes herself available to building principals in the district when needed. The SRO supervisor's role in the creation, implementation, and sustaining of the school emergency management plans is somewhat limited due to having assignments that take priority within their respective law enforcement agencies.

In RHS \#2, Sally is assigned as an SRO to a set of schools while fulfilling her duties as the SRO supervisor. In her capacity as a supervisor, she must ensure that the SROs under her supervision carry out their assigned duties at their respective schools while also handling her own schools as an SRO.

Not one of the SROs in the three high schools in this study had any input into the creation or implementation of their respective school emergency management plans, but each plays a major role in the sustaining of their respective school plans. In all three schools, the SROs have had a role in the scheduling and completion of the drills and exercises associated with the safety 
plans. In two of the schools, the SROs work primarily with the assistant principal or deans at their buildings while in the other school, the SRO works directly with the building principal.

Both Ronald and Roger are SROs specifically assigned to their respective high schools, but only Roger attends all of the school administration meetings while Ronald is invited periodically if the principal believes it is necessary. On the other hand, Rodney is assigned primarily to the high school but must also answer calls for service at the middle and elementary schools. At the beginning of this school year, the principal made the decision to include Rodney in their administrative meetings, and she has observed positive results in their collaborative efforts.

As part of the cross-case analysis, reviews of the interorganizational relationships across the districts were studied to determine the existence of the school and law enforcement partnership in school emergency management. Interorganizational relationships have the potential to be fluid, dynamic, and at times quite difficult given the differing perspectives and cultures within the individual entities. Differences between school and law enforcement personnel are no different given the vast differences in mindsets and objectivity. Schools and law enforcement agencies have collaborated for decades with the goal of keeping students, staff, and community members safe on school grounds. The dynamic relationship between school and law enforcement personnel in this study demonstrated natural barriers within the partnership depending on the individual roles.

In this study, two of the participants expressed concerns during the change of leadership at their respective law enforcement agency. One participant was unsure if the new police chief would support the SRO program in their district and thought that the SRO program would be pulled. Ultimately, the new chief was an avid supporter of the SRO program. Darryl reported 
that with each new police chief, he has had to go through the process of "introducing" the SRO program to garner continued support. When a change in leadership occurs either at the school or law enforcement administrative level, there stands a possibility of losing support for the SRO programs.

Participants described other obstacles during the interviews regarding specific roles they have within their individual school sites. Peggy asked rhetorically, "What is the role of the SRO?" Of course, as a principal, she has a general idea of the role of the SRO but feels that a better line of communication is necessary to discuss specific details about their daily functions. Conversely, Roger who is the SRO revealed that he sees himself as a liaison between the police department and the school. He also noted that he also plays other roles such as a mentor, counselor, and teacher. Roger shared, "I am not always dealing with bad kids. I also deal with good kids." His assignments or duties vary depending on the need of the school. It is clear that there is miscommunication as to the specific role of the SRO at this school site, but this a shared instance among the other cases, as well.

Rodney discussed some of duties as "going from school to school, educating students on everything from drugs to alcohol, cyber-bullying, you name it." He expressed that it is important for people to know that they are there for positive reasons as well. It is difficult to pin down the exact expectations of an SRO with all of the dynamic features attached to this type of assignment.

Pamela said that in her role as a principal, she has had three SROs in six years. She understands the need for a change in law enforcement assignments; however, she asserted that it would be beneficial to have an SRO for a longer period, which would allow them the opportunity to get to know the students and staff. Daniel had similar thoughts and explained, "Relationships 
are formed, and they go away." He noted that it is not just turnover with SROs, but it is also observed with transitions in school administration.

Dwight and Patricia relayed concern about the multiple schools that the SROs in their district must serve, which has taken them off-site when they are needed. Rodney agreed, "There is just not enough time [to get everything done because he has to] cover four schools with four different principals." Dwight acknowledged that he would like to see SROs and school administrators attend training together to have opportunities to collaborate beyond the typical workday. He said that he has "established his best relationships - going to the beach, eating, talking with those folks — outside of the school setting," during a training conference. Patricia added, "We need to trust each other by working together awhile to work out the kinks in a relationship." According to several participants, there is a need for school and law enforcement personnel to spend time together to develop an effective relationship.

Another hindrance in this type of interorganizational relationship is the basic philosophy of deciding which options to choose in given circumstances. As Roger explained, "We have disagreements on how certain situations should be handled." Part of the reason he asserted is that school administration does not fully understand criminal law, which creates misunderstandings. Samantha had similar sentiments; she added, "[School and law enforcement] are different organizations: they are education and we are law enforcement ... we have to find a middle ground." She also acknowledged that it is important to try to understand the other's goals, but in turn, it should be reciprocated. Samantha added, "When it comes to school discipline, we need to take a secondary role.”

One of the SROs in this study shared, "The school sees me as a guest. I am here to support but not necessarily as part of the staff," while his department sees him solely in a law 
enforcement role. Ideally, in any organization, it is preferable to have one supervisor but in the case of SROs, they typically must answer to their law enforcement supervisor and building principal. For example, one principal discussed this problem and said, "Although they are police officers, there are people in the building that trump them. I think they (SROs) have a hard time understanding that." She understands that law enforcement prioritizes situations differently, and having differing perspectives is okay as long as they end up on the same page.

\section{Within-Case Synthesis}

As previously mentioned, the three school districts in this study are located in the Midwest, South, and Southwestern regions of the United States, which make it mandatory for each public-school district to have school emergency management plans that include regularly scheduled emergency drills. Each of these states provide emergency plan templates to use in developing school emergency management plans; however, in some cases, it is not mandatory to use the template as long as the school emergency management plans fit the criteria expected by each state.

Two of the school districts in this study used their state emergency plan template but tailored each school emergency management plan to meet the needs of each school, pre-k through high school. For example, one state mandates that one of the districts undergo a threeyear review. Pamela said, "Three years ago the state came out with a template change, and that's when we had to go through a review and resubmit it," meaning the school emergency management plans. Through that process, the district had to review and assess their current school emergency management plans to demonstrate adherence to the standards set for by the state education agency. 
Conversely, another district voluntarily decided to update its school emergency management plans completely to create an improved system. As Dwight stated, "We have basically started from the ground up. We scrapped everything and started over." As part of that process, the district put their school emergency management plans into a digital format through their state education agency. The state digital system allows each district to show their school emergency management plans, training, drill schedules, and school maps, which can also be accessed by law enforcement officers in the state through a software program.

The third district in this study created its plans in conjunction with a nearby city fire department that received a federal safety grant. Darryl explained, "The current district school safety crisis plan began development [of the plan] about 15 years ago through a grant." He reported that an all-hazards plan was developed for area schools based on DHS, FEMA, NIMS, and ICS concepts.

School safety training for administration, staff, and SROs is mandatory at each of the districts that participated in this study; however, the training is not the same at all three sites. One district requires school administrators to attend school safety courses provided online by FEMA. Darryl said, "Several years ago all of our administrators had gone through the emergency management institute (EMI), which is FEMA's online training system for NIMS and ICS for schools." He also noted that due to attrition at the school building administrator level, newly-hired administrators must complete the EMI course this year.

Pamela, in describing part of their training program, said that the district SROs and their respective law enforcement agency created a video series on the school lockdown process to use in training school administration and staff. The goal, according to Pamela, is to compile a series 
of safety-related training videos for "emergency response crisis training." The online video format makes it easier for each staff member to obtain the needed training.

Dwight reported that his school district recently added current staff members to act as safety liaisons for each school. Safety liaisons represent their campuses at the monthly safety meetings and are familiar with their respective schools. The liaisons assist with emergency drills and the after-action reports that pertain to school safety that are discussed in the monthly safety meetings.

Sustaining school emergency management plans requires a regular assessment of the written plans, emergency drills, and current training methods. Assessing a drill requires observers from the schools, law enforcement agencies, and fire departments that typically have jurisdiction over the school district. Once the drill is completed, an after-action meeting takes place to evaluate the positive and negative results of the emergency drill. The information derived from the after-action meeting is then relayed to the participants of the drill to either modify or preserve the current safety procedures. When deficiencies have been identified through this process, further professional development may be implemented by the school district.

School districts in this qualitative multiple case study use methods to sustain emergency plans by using debrief type meetings after emergency drills and exercises. As an example, Darryl said, "Immediately following a drill or actual event, administrators complete an afteraction debrief of the event." Similarly, Sally described the after-action process in her district as "sitting down with school administration and discussing what happened during the drill and identifying items for improvement." According to Daniel, each of the SROs have a checklist for 
their respective school and before the beginning of the school year to ensure that staff at that school are well versed and reminded about their school emergency management plans.

\section{Themes}

Several themes emerged from interviews, documents, and artifacts from each of the three school sites and the respective participants. Initial analysis of the interviews resulted in 25 codes that were garnered from the answers to questions related to interorganizational relationships between school and law enforcement personnel and school emergency management processes. Next, the 25 codes were narrowed down into five major themes Communication, Networking, Mutual Understanding, Priorities, and Training (see Appendices D and E).

\section{Theme One: Communication}

All of the participants in this study reported communication as being one of the primary aspects of school emergency management plans and their working relationship with their immediate counterparts. The prevailing line of thought among the participants is the importance of having the opportunity to meet and discuss various issues related to safety.

Communication within the participants' respective districts has both positive and negative aspects. Patricia, a principal, and Rodney, a SRO at RHS \# 2, discussed their willingness to talk anytime, day or night. to help resolve problems associated with school safety and discipline. Conversely, Peggy, the principal at RHS \# 3, mentioned the difficulty in finding time throughout her busy schedule to have discussions with the SRO.

For district administrators, communication is of major importance. They are responsible for school safety within their respective districts, which happens through all phases of school emergency planning. Each of the district administrators in this study is responsible for 
establishing school emergency management plans and ensuring that the district meets minimum standards expected by the state education agency.

Dwight reported that he establishes communication through monthly meetings with safety liaisons and building principals to discuss school emergency management plans as a district and for individual school sites. Daniel acknowledged that he primarily communicates with building principals and the SROs. He proclaimed that the best way to establish a strong working relationship is to have "constant communication between the district, SRO, and building principal." As a former principal, Pamela asserted that improving her relationship with the SRO requires daily opportunities for communication.

The fact that SROs do not have a "centralized command system but a school-based one," causes a lack of information sharing according to Darryl. To overcome this communication obstacle, monthly meetings supervised by Darryl have been initiated and have met with some resistance initially. However, after a couple of months, some trust was established, and communication flowed more willingly. Darryl surmised that since the inception of the monthly meetings, the "floodgates opened and commonalities were established." Two of the three district administrators established that working together to handle safety issues and having constant communication creates a system of comradery and trust.

At RHS \# 3, the assistant principals handle the primary communication with the SRO. Peggy understands that communication with the SRO is necessary although finding the time given the responsibilities of a building principal has proven to be difficult. She said, "Making to time communicate with the SRO has to become a priority." Peggy reported that sitting down and talking to share each other's viewpoint on school safety helps to find a middle ground and establish a mutual understanding. 
According to Pamela, building a consensus among stakeholders involved in school safety requires improved communication to involve all stakeholders. In order to make a rational and fully informed decision regarding a case of student discipline and whether or not it meets the criteria of a criminal act, she has to sit down with the SRO and discuss the options. Pamela explained, "We are just going to come together and say, 'What way do we need to go?"” Likewise, Patricia prefers "talking it out to come to a consensus" with the SRO when it comes to making decisions about student behavior that may be considered a criminal act.

\section{Theme Two: Networking}

According to the participants, meaningful networking and collaborative practices for organizations that are involved in an interorganizational relationship are critical components of a successful endeavor. Successful education/law enforcement relationships require on-going collaboration and networking to navigate through the myriad of issues related to school safety. Networking within these organizations comes in various forms whether it is a part of a training conference, a scheduled meeting, or ad-hoc situations that requires discussion to mediate a solution.

Successful networking in interorganizational relationships does not just happen, it takes time and work to build a level of comfort, trust, and comradery as reported by all the participants in this study. Dwight said, "It takes time for them to start to trust you and for you to trust them because a lot happens behind closed doors." He noted that much if not all of the discussions between school and law enforcement employees pertaining to the safety of staff, students, and community members must remain confidential. Establishing trust and openness to be able to discuss some of these potentially life-changing decisions requires a high-degree of confidence in each other. 
Sharon talked about the networking process as being "a group of stakeholders sitting in a room and putting the problem out for everyone to hear. Next, those within the group offer suggestions and solutions until we find a viable resolution." Patricia described, "It's not a dictatorship ... it's let's sit there and talk about what our needs are and how we can work this out." Pamela added, "[When a] big decision needs to be made, we're going to sit down at a table and all get on the same page." Sharon purported that she prefers to discuss everything and provide an explanation for her decision and not simply assume that the others in the group understand her reasoning.

The participants argued that other methods of developing a strong, collaborative network occur through regular meetings before, during, and following the school year. For example, Daniel reported that at the beginning of each year the county puts together an administrative retreat called a Safety Summit. Administrators from each school district within the county along with law enforcement personnel are invited to attend the summit to share knowledge and discuss various safety related issues. The other schools in this study have similar meetings, but as Darryl stated, "Getting all of those people in the same room at the same time to be on the same page took a couple of years." He also reported that their collaborative efforts have led to familiarity among the group members, which eventually has created a sense of trust as partners.

Having SROs regularly attend school administration meetings may not be a typical practice. Patricia discussed a recent change in having the SRO attend the weekly leadership team meetings; a difference is also evident in the amount of cooperation and buy-in with their school mission and objective. She said, "The more meetings that we're at together, the better off it will all become," referring to the relationship between school administration and law enforcement. 
Roger discussed the idea of attending weekly meetings with school administrative staff and how doing so contributes to the relationship. He stated, "I work to be on the same page with the dean; I listen to them and they listen to me." All of the participants discussed the importance of networking and collaboration as a way of getting through their differences. Sally added, "You need communication to bridge that gap." Patricia described the value of meetings as providing an avenue for networking by saying, "The more meetings that we're at together, the better off it will all become." Rodney surmised, "It's all about working together; that's our main goal, to work together to ensure safety first." Peggy concluded, "Finding common ground, having dialogue about what we ultimately want to accomplish, what we feel [comfortable] doing or giving up, [and coming to a consensus]" is a process.

\section{Theme Three: Mutual Understanding}

In interorganizational relationships between schools and law enforcement personnel, it is imperative to understand each other's perspective, role, or policies in school safety. However, this type of mutual understanding is not always a standard practice according to the participants. One of the SROs in this study described this plight by reporting that his view is that schools have an entirely different agenda than law enforcement. Educational performance goals create an impasse in school safety at times.

Samantha had a similar perspective and said, “They don't fully understand what police policies and procedures are, and at the same time I don't understand their policies and practices." She reported that it is an ongoing, evolving procedure to understand each other to avoid miscommunication. Sharon further speculated that school personnel in general has a different philosophy than those in the police department in many areas because, "it's a different organization." As one principal stated: 
SROs look at school safety from a police perspective and sometimes they think a situation is really bad, but I prefer to talk to the student first to see if I can handle the matter within our school disciplinary process. I look at it from a softer side because I can work with the kid, whereas SROs look at it from criminal law perspective. Police are used to taking charge but the way I see it is this is my school, and I am ultimately responsible for what takes place in my building.

Pamela described her perspective by reporting that an incident will occur and the assistant principals or principal have to determine if a law has been broken or if it falls under school discipline. "Sometimes the two kind of blend into one another, she said." Pamela concluded, "in these circumstances, we have to sit down with the SRO to help make a determination."

Samantha described one of the differences in perspectives by saying, "Teachers [and] school administrators go into schools and their beliefs are kind of daisies and roses, but they don't see the bad things." Rodney described the difference in perspective by saying that, "we go to worst case scenarios." Roger provided another perspective of this dynamic when he explained:

I am not a social worker, I am a police officer, so obviously I think a lot different[ly] than a dean. We have a different mindset. According to one SRO, the lack in mutual perspective has led to being asked to do something that cannot be done by law. Other times, I want to do something that they do not want done.

Similarly, Sharon added, "The two have different goals, one side may not agree with the other, but it is what it is and at the end of the day you try and work it out the best you can."

According to the Patricia, some of the misunderstanding in this relationship results from the dual roles that SROs must fulfill. Sally described this dynamic saying, "The police 
department knows we work for them and you are assigned as an SRO but before anything you are a certified officer first." If the police department needs her to handle emergencies outside of the school, she has to respond accordingly.

Darryl added a different perspective. He said, "I told the chiefs [that] they are still your officers, but when they are here during the day they are really my guys and I need them to operate as though this is a school district police department." Dwight shared, "If both groups can understand and know where the lines are drawn ... it's the idea of folks letting go and being able to collaborate together." Through this discussion, two recommendations emerged. First, Dwight stated, "SROs and school administrators should attend training together whether it is an educational or a law enforcement conference to gain a mutual understanding." Second, Sharon added, "We have to be willing to bridge that gap." She also suggested that SROs should review the school handbook to gain an understanding that schools are bound by a set of guidelines. The majority of participants agreed with the idea that understanding each other's perspective and roles will enhance their relationships.

\section{Theme Four: Priorities}

SROs are in an untenable position because of the dual role they have as both a police officer with their respective law enforcement agency and their assignment to a school with another quasi-supervisor. In certain events, the participants asserted that the SRO must decide between (a) staying on campus to ensure the safety of students, and (b) leaving campus to respond to an emergency in the community.

One of the principals reported experiencing situations when their SRO left campus to handle an off-campus emergency, which left the school vulnerable in the aftermath of the same incident. Sally described this dilemma by saying, "When it comes to the police department 
needing me, [I'm] torn between the two [and I] feel like [I'm] being pulled in several different directions." In similar fashion, Rodney, who is assigned primarily to the high school, could be pulled away to handle a situation at any one of the other four elementary schools under his supervision.

Similarly, decisions have to be made when the police department needs the SRO to attend certain training or the school would like to have an SRO attend their training but supervision at the police department level does not allow the time off. Darryl described occasions when he wanted an SRO to attend a particular training conference but the police department said that they could not release them for the training. Conversely, Pamela replied, "The police department needed the SRO to attend particular training which left the school without coverage." As Darryl stated, "I can suggest a lot of things, but it's really up to the departments."

Another difficult compromise in the relationship between law enforcement and school is deciding when additional meetings, trainings, and related safety programs should occur. Administrative participants noted that their primary focus is education, which covers a wide range of responsibilities. For example, school administration must make time for testing, data analysis, faculty meetings, faculty evaluations, meetings with parents and community members, attending district administrative meetings, and after-school and weekend events among others. Within this active daily routine, the principals in this study acknowledged that there is often not time to build relationships with SROs and other personnel related to safety.

In one interview, Peggy asserted there must be time to share issues and said, "As a school, you just get going, and you don't have time to bring the SRO into the mix." She reported that it is a matter of setting aside time and making it a priority to meet. However, the real question is where do you find the time while school is in session? Pamela, responded, "We need 
to take time out of our regular professional development, which is tough when our focus is on learning." These principals asserted the importance of relationship building with the SROs and focus on safety matters, but it comes down to deciding on their priorities. One way of finding this time, according to Patricia, is to have the SRO attend the weekly school leadership meetings. She commented, "The more meetings and trainings we go to together, the better it will all work."

The perspective of the SRO is similar to the principals with this dynamic. Ronald reported that he has daily informal contact with various school administrators but, "I would love to do more, there's just not enough time." He added that activities outside of the classroom inundate school administrators, which make it difficult to get time to discuss safety with teachers. Ronald concluded, "There is no time to accomplish all of the law enforcement objectives plus all of [the] safety objectives and school objectives and find time to participate in all of the activities that I would like to participate in."

Rodney provided similar sentiments when he said, "Schools have so much to get done, testing, making goals each month and year, but you're only allowed so much time." Samantha added, "Finding windows of opportunity to do drills, after-action meetings, and [getting] everyone on the same schedule can be very challenging." While the principals and SROs in this study all agreed that building relationships and spending time together is crucial in building a solid, working relationship, finding the "windows of opportunity" to train and conduct meetings proves problematic.

\section{Theme Five: Training}

In this study, training referred to meetings between school and law enforcement personnel, large-scale exercises, tabletop drills, emergency drills, and actual emergency events. Actual emergency events are included as a part of training process because of the practice of 
completing after-action exercises to review their performance and make the necessary adjustments. Training in this context can also include online and face-to-face courses.

Each of the schools in this study is housed in states that require a number of emergency drills each year. These drills include fire, lockdowns, severe weather, active-shooter, and reverse evacuations to name a few. Most of the participants discussed mandatory drills as related to their individual school sites. Darryl noted that the state education agency requires assessments but also reported that they add additional emergency drills to ensure readiness. One particular state requires one drill per year to be conducted alongside of law enforcement. Darryl's district executes three of these per year because in his opinion this is an area of needed improvement.

Dwight had similar thoughts regarding state mandated drills; however, when the drills are completed in his district, the results are uploaded into a state software program. He reported that the state mandates how often the various types of drills will be performed, but when and where are somewhat flexible. Sally explained, "The police department completes training each month to include, fire, intruder, and inclement weather drills, and [RHS \# 1] is required to have nine drills throughout the year." Samantha described how the SROs in her police department assist school administration by monitoring the drills and ensuring that everyone follows policies and procedures implemented by the district. However, as one of the SROs said, "The only thing we do are mandatory drills, and even those do not seem very effective."

As far as the actual emergency drills, each of the schools in this study has its own unique way of completing them; however, there are also similarities. In describing emergency drills, Pamela said, "Emergency drills are [the] most effective training method." She said that most of the staff members have similar reactions in an actual event as they have in the drills, which speaks enormously to the reason of practicing through emergency drills. Pamela shared, "Even 
though we have implemented the use of technology through online methods to do some training, it is difficult to find to the time train because the district is focused on learning." She talked about a fire drill in which "the principals were running around clearing buildings, but that is not their role: they should be directing personnel." Pamela added, "We learned to change our methodology by making the principals a part of the central command." She also disclosed that they now have security guards and other staff members to assist with clearing the buildings.

Daniel surmised that emergency management readiness comes down to continual practice and ensuring that school safety remains a top priority. According to Dwight, "They have done a lot of training with our staff members and different groups in order to improve our practice." Sharon described conducting a large-scale drill annually along with other first responding agencies. The training takes a full-day, and individuals perform as role players to make the drill more realistic. In similar fashion, Rodney added:

We have to train, train, train. It takes constant training, as we learn something new each time we conduct a drill. You have to gain their trust [and] learn to trust each other, through the process of training and working together.

Samantha acknowledged that their training method includes those involved learning which staff member makes the 911 call, communicates with students, contacts other high school administrators, and communicates directly with law enforcement.

As previously mentioned, after-action meetings are methods of reviewing the drills to look for what those involved did correctly and which areas need improvement. Rodney likened the after-action process at his district by stating, "It's like a football team: we play on Saturday [and] tomorrow we come into a locked film room to see what we can correct or what we can make better." Darryl described the after-action process by saying, "We do an after-action 'hot- 
wash' debrief of each scenario." In his district, the participants in the drill expect to have a meeting in the main office after a fire drill, lockdown, or a real event. Darryl asserted that debriefs are essential to improving the next time. According to Dwight, they sit down with the principal or team members and go over the events of the drill to figure out what needs to be improved. "When we meet after a drill, everyone brings an individual report for further analysis," he said.

Each component of training such as meetings, emergency drills, large-scale exercises, tabletop drills, and after-action meetings is as important as the next in creating an atmosphere of school emergency management readiness. Each of the schools in this study uses these training methods as a part of a regular practice and opportunities for improvement.

\section{Answers to Research Questions}

The research questions were developed from the literature review regarding issues of interorganizational relationships between school and law enforcement personnel in developing school safety plans. The central question (CQ) sought to discover how district administrators, building principals, SROs, and SRO supervisors perceive their individual roles in the context of school emergency management. Together, the sub questions (SQ) sought to explore specific factors that are contained within the central question. The answers to the sub questions combine to provide an in-depth answer to the central question and were answered through the five themes that materialized. Codes were identified from the data analysis process, which methodically narrowed the list of codes until the emerging themes were developed.

CQ: How do school and law enforcement personnel in the Midwest, South, and Southwest regions of the U.S. collaborate to create, implement, and sustain school emergency management readiness? School and law enforcement personnel have distinct 
cultures in the way that they approach their unique vocations. Most states require that teachers hold a bachelor's degree and specific training on classroom instructional methods. Additionally, most principals must have a master's degree_-most frequently in educational leadership —along with completing an internship to become an instructional leader. There is no set amount of time teaching that building principals must have before moving into administration but typically, it is a minimum of five years. Building principals typically serve as an assistant principal or dean first before moving and taking the lead role in a school building.

On the other hand, most police departments require anywhere from a high school diploma, high school equivalency, some college, or in rare instances, a bachelor's degree. Once hired, most police officers receive training through a police academy followed by one-on-one training on the job with a field-training officer.

Most SROs typically have a few years of experience assigned to patrol before working in a specialized unit. In the case of SRO supervisors, they typically have served a certain number of years in law enforcement before moving into supervision. In this qualitative multiple case study, each of the SRO supervisors is a first-line supervisor, which is either a sergeant or lieutenant.

Each participant's role in this study had a distinctly unique path to his or her current position. It makes sense that the cultural differences between school and law enforcement personnel are boundless. Additionally, the mission objectives for school and law enforcement personnel are unique to the role that each serve within the school. Customarily, the mission objectives for educational personnel within a school system are to provide educational opportunities for students working toward the goal of high school graduation. Conversely, law enforcement's primary mission objective is to enforce the laws. SROs are in an atypical 
situation in that they work under the supervision of two organizations with unique cultures and mission objectives that do not consistently have the same goals.

Darryl defined the differences of the mission objectives by reporting that the school's primary mission is to serve the students and ensure that they meet students' academic needs. Part of the objective is to provide educational opportunities for the community. He posited that school and law enforcement provide a social service to the community, which creates some cross over between the two organizations. Darryl said, "We operate independently of each other because of the nature of our jobs, but in the end, it's a uniform approach to service in the community." With a slightly different perspective, Pamela divulged that her primary mission is to provide an education while law enforcement handles emergency management. She recalled:

Our thinking has transitioned to more of a joint command during emergency incidents. The assistant principals or myself handle the faculty and staff. Law enforcement supervision handles their officers but we work together in the decision-making process.

Dwight had similar reflections saying, "Our mission is to teach and keep everyone safe while law enforcement makes sure that we are following the laws. It is important that we work together toward the same objective."

Commensurate to what has been discussed, Dwight said that the school and law enforcement are on two completely different paths, but their goals go hand-in-hand. He concluded, "[When] we create a safe learning environment then student learning takes place." From a law enforcement perspective. Samantha said:

Our number one goal is that no one gets hurt and everybody goes home. Even though we run on parallel tracks and are headed in the same direction, school 
administration has different policies and procedures, which is why it is important to train together.

Similarly, as stated by Sally, "Our goals, rules, and regulations differ, but we have to work together toward a common goal." Rodney shared, "At the end of the day, the mission objectives for the schools and law enforcement are about working together, that's our main goal, safety first."

Most of the participants had similar viewpoints when discussing the idea of having differing mission objectives. In the day-to-day working environment between school administration and SROs, according to Pamela, "There's a lot of nuances" in this relationship. For example, she explained:

We may have a principal dealing with a student and have to determine if a law has been broken or if it just a school discipline type of event. At times, the two situations, criminal and school discipline, blend together, and we have to work through the problem. In these types of cases, our SRO usually walks us through the decision-making process.

To put it more succinctly, Patricia said that in most cases, "they talk it out but if it is a true emergency, law enforcement takes the lead; other than that, we will determine what is going to happen in our schools." Samantha stated, "If there is an emergency involving an armed intruder or gunman, we will take charge and school administration will have to take a step back. However, when it comes to school discipline, we take a secondary role." Roger added, "As a way of reviewing our performance as a team, we have a meeting at the end of the year to evaluate our system." He concluded, "At times we disagree, but it does not turn negative; we work to find a happy medium." 
The interorganizational relationship between school and law enforcement personnel with differing missions plays a key role in creating a safe environment for students and staff. In accomplishing this task, most of the participants agreed that the two entities must have an effective communication process to work through their differing viewpoints and perspectives.

\section{SQ1: How do personnel from schools and law enforcement create school emergency}

management plans? The three high schools participating in this qualitative multiple case study all have similar approaches to creating school emergency plans for their respective districts. Each school through its district has adopted its respective state education agency's recommendations in creating their school emergency plans. However, Darryl, did use other sources in developing their plans by joining in with a local fire department that received a FEMA grant. He worked closely with the local fire department to create school emergency management plans based on FEMA and DHS guidelines. Through the collaborative efforts over a 15-year period, the district developed a district-wide, all-hazards school emergency plan that was adopted by neighboring school districts.

Through their position within the district and as the liaison with their respective state education agencies, district administrators perform an important role in the creation of school emergency plans. As part of their responsibility as the designated district administrator in charge of school safety, it appears that they follow the lead provided by the state education agency. At the district level, two of the high schools in this study used the safety plan template provided by the state education agency but tailored the plans to meet the unique needs of their schools. The other school and district specifically utilized FEMA and DHS guidelines.

Other participants such as the principals, SROs, and SRO supervisors explained that they do not contribute to the creation of school emergency management plans within their districts. 
Two of the principals were knowledgeable about the historic creation of the plans while the other principal did not because he was relatively new to the district. At the same time, two of the SRO supervisors reported having a solid working knowledge of the creation of the school emergency management plans within their district. According to Samantha, schools write emergency plans at the request of the state education agency, but when the schools have questions or concerns, they help as consultants.

\section{SQ2: How do personnel from schools and law enforcement implement school} emergency management plans? Implementation of school emergency management plans is a process whereby each school district takes the set of written emergency plans and puts them into practice. Each of the three schools in this study differed in their approach to implementing their respective school emergency management plans but, similarly to the creation of school emergency management plans, district administrators play a significant role in the implementation process.

Darryl described the implementation process by reporting that once all of the stakeholders come to a consensus on the written plans, the superintendent has to adopt them with the approval of the board of education. He added that having a district plan solved a myriad of problems due to building principals having to manage their own plans. Similarly, Sally expressed her thoughts on their district implementation development noting that the district underwent approval through the superintendent and school board.

From a different perspective, Daniel viewed the implementation of school emergency management plans as a yearly event in which school and law enforcement personnel have an opportunity to foster their relationship. He disclosed that the SROs go through a checklist with educators as reminders that ensure everyone is well versed on the school emergency 
management plans. Additionally, each year has new requirements and changes in the statute or school emergency management plan policies. This process allows everyone to make the necessary adjustments as needed for their individual school emergency management plans.

Pamela revealed that their yearly implementation process is organized by having assistant principals lead safety discussions with faculty and staff. As a group, they discuss the various types of counteraction to various types of emergencies. As part of the training, each teacher receives a key chain that contains information on what to do in the event of certain emergencies, along with key contact phone numbers. Pamela also reported that the SROs and SRO supervisors address response methods with faculty and staff regarding various types of emergency events.

As part of the implementation process, Sally stressed the importance of working together to refine school emergency management plans and putting them into practice. Dwight discussed a digital application that provides a linkage to the state education department. This particular digital application gives each district the opportunity to demonstrate its emergency readiness by answering a series of questions through an online format. Dwight noted that the application is shared with local first-responders who can access instant information as to the nature of the problem, school maps, and persons to contact.

Each school district in this study has an implementation process for its respective school emergency management plans. Similar to the creation of school emergency management plans, implementation appears to be the primary responsibility of district administrators who in turn work with each of the building principals, SROs, and the SRO supervisor to ensure that the school emergency management plans are put into practice. 


\section{SQ3: How do personnel from schools and law enforcement sustain school}

emergency management plans? Mandatory or required monthly emergency drills stand out as one the most significant methods of sustaining school emergency management plans. For example, the majority of the participants discussed the realization that respective state education agencies require each to conduct a minimum number of emergency drills per month, including fire, severe weather, intruder, and evacuations. Depending on the particular school, the principal, assistant principal, dean, or SRO ensures that their respective school building is in adherence with mandatory state drills. Furthermore, each of the districts schedules annual safety meetings to discuss the drill itinerary for the upcoming school year.

RHS \# 3 conducts their annual meetings in collaboration with local fire, police, emergency dispatch, area hospital, and other interested parties. Stakeholders in this meeting discuss the current school emergency management plans, what occurred over the past year, and which items they would like to change for the upcoming school year. As a regular practice, RHS \# 2 and RHS \# 3 conduct safety meetings throughout the school year. As Dwight explained, "Recently, we scrapped our school emergency management plans and started over ... we developed a committee with safety liaisons from each school so we can meet and discuss safety matters." According to Darryl, he conducts weekly safety meetings throughout the year to discuss safety issues and conduct tabletop exercises two to three times a year. He added that every few years, building administrators are responsible for taking online FEMA school safety courses as a re-certification process. As part of the process of sustaining school emergency management plans, Pamela and Daniel mentioned that their local police agencies developed a series of video training seminars related to school safety with the assistance of the SROs. 
Additionally, RHS \# 1 participates in a countywide emergency management training to discuss safety related issues in their area.

As a whole, the SROs and SRO supervisors play a supporting role in school emergency management by acting as observers, consultants, and trainers. Samantha characterized her role as an observer to confirm that everyone participating in the emergency drills is following policy and procedures. She said, "We then have after-actions to discuss the drills, what we did good, what we did bad, and what we can do better."

\section{Summary}

This chapter provided the results of the experiences of 12 participants from one rural and two suburban high schools located in various regions throughout the United States. It included findings from the data that explain the interorganizational relationships between school and law enforcement personnel in the creation, implementation, and sustaining school emergency management plans. The purpose of this qualitative multiple case study was to explore and understand school and law enforcement personnel perceptions on the interorganizational relationship in the context of school emergency plans. The participants included district administrators, building principals, SROs, and SRO supervisors. Face-to-face interviews, analysis of relevant documents, and within-case and cross-case analyses identified the perceptions of school and law enforcement personnel. The within-case and cross-case analyses also provided and in-depth examination of the perceptions of each of the participant from each his or her unique roles in school emergency management. 


\section{CHAPTER FIVE: CONCLUSION}

\section{Overview}

The purpose of this qualitative multiple case study was to examine the interorganizational relationship between school and law enforcement personnel in the creation, implementation, and sustaining of school emergency management plans in three high schools located in Midwest, South, and Southwestern regions in the United States. Given past, recent, and potential for future armed attacks, natural and technical disasters on or near a school has provided a basis for this examination. This study provides a significant contribution in relevant literature to assist current and future school and law enforcement personnel in their interorganizational relationships as they pertain to school emergency management readiness. The findings and implications of relevant literature and theoretical concepts, delimitations and limitations, and recommendations for future research are presented in this chapter.

\section{Summary of Findings}

Data was collected for this study from interviews conducted with 12 participants-four from each school site - which consisted of a district administrator, building principal, SRO, and SRO supervisor. Following individual interviews, documents, and artifacts from each school site, data was compiled and downloaded into ATLAS.ti. Next, the data was analyzed to aggregate codes into emergent themes. Themes that emerged from the original list of codes that are directly related to each of the research questions were Communication, Networking, Mutual Understanding, Priorities, and Training. The use of within-case and cross-case analysis along with a thorough examination of findings from this study provides future researchers a baseline to further explore answers to research questions in consideration of developing or creating an effective school emergency management plan. 


\section{Central Research Question and Sub Questions}

The central question driving this study asked: "How do school and law enforcement personnel in the Midwest, South, and Southwestern regions in the United States with diverse cultures, missions, and objectives, collaborate to create, implement, and sustain emergency management readiness?" All of the participants agreed that differences exist among their respective cultures, missions, and objectives. However, there is a standard thought among the participants that through effective communication, collaboration, and agreed upon goals, these differences can be overcome in creating a safe school environment.

The analysis also showed that significant differences exist due to certain constraints. For example, one of the principals discussed the lack of available time during a school day to sit down and share ideas with their SRO. Another example, provided by a district administrator, was the problem associated with SROs being supervised by their law enforcement agency and school administration, which creates a dilemma for the SRO. Moreover, all of the SROs agreed that on-going training for emergencies should be a high priority, but there is a shortage of opportunities because of the educational priorities that trump emergency readiness.

This first sub question asked: "How do personnel from schools and law enforcement create school emergency plans?" The overall resounding answer to this question centered on the fact that district administrators from each of the respective school sites played a major role in creating their school emergency management plans. The two district administrators admitted using the state educational agency template for the school emergency plans, while the other district administrator worked closely with a local fire department to create an all-hazards type of approach which models FEMA, NIMS, and ICS concepts. It was surprising to learn that local first-responders, police, and fire did not have a hand in the creation of the school emergency 
plans at two of the sites. However, the state education agency may have collaborated in consultation with fire and law enforcement personnel in creating their school emergency plan templates.

The second sub question asked: "How do personnel from schools and law enforcement implement school emergency plans?" This answer varied among the participants depending on their individual definitions of implementation of school emergency plans. For example, one of the SROs from this study discussed the process of creating the plans and then going through the superintendent and school board for final approval as part of the implementation process. On the other hand, all of the district administrators viewed this process as handing the plans down to the principals who in turn put their plans into action at their site. Additionally, the process across this study showed that more of the participants between school and law enforcement personnel are engaged in the activities that lend itself to setting up and ensuring that the plans were in place.

The third sub question asked: "How do personnel from schools and law enforcement sustain school emergency plans?" The process of sustaining school emergency management plans requires actual practice of carrying out the plans through emergency drills, table-top exercises, and large-scale multi-jurisdictional exercises. Overall, the principals and SROs seemed to take the lead in planning and completing emergency drills. The role of the principals and SROs in this process is one of overseeing and observing the drills as active participants. In turn, at the completion of the drills and exercises, principals and SROs lead discussions with the participants to talk about the positive and negative results of the drill. The participant administrators noted that each of the school sites reports the completion of the drill to their respective state educational agency. 


\section{Discussion}

Empirical evidence from this study testified to the literature review regarding the effect of the interorganizational relationship of school and law enforcement personnel in the creating, implementing, and sustaining of school emergency management plans. The discussion begins with the empirical and practical examinations of this study. Next, an investigation of the theoretical findings is shown through multiple definitions of interorganizational relationships, which provides a generic framework shared by authors such as McGuire and Silvia (2010) and Reitan (1998).

\section{Empirical Foundations}

There is a limited amount of research available on disaster preparation of emergency management for schools and the collaborative efforts between stakeholder agencies, namely school and law enforcement personnel (Cray \& Weller, 2011; Kano, \& Bourque, 2008; Kapucu $\& \mathrm{Hu}, 2014)$. According to the Council of State Governments Justice Center (2014), 33 states have specific laws that require every school or school district to have a comprehensive school emergency management plan. Additionally, approximately $56 \%$ of the schools in the U.S. have school emergency plans that follow federal government school emergency management protocols (Kano \& Bourque, 2008). Furthermore, fewer than 20 of the 50 states have an agency whose primary focus is school emergency management (Hull, 2011). In this study, each of the schools has emergency plans and incorporates drills, meetings, and training exercises to maintain readiness.

The central research question for this study focused on the participants' perspectives in relation to the process of school and law enforcement personnel with diverse cultures, missions, and objectives collaborating to create, implement, and sustain school emergency management 
plans. Participants in this study provided a myriad of observations through the lens of their role within their respective school district. Coon and Travis (2012) opined that the development of school emergency plans should be a joint venture involving school and law enforcement personnel. While the relationship between district administration and SRO supervision is important, this study showed that the number of meetings between the two entities is limited and spread out through the year.

As part of the school emergency management plan process, it is imperative that school administrators take an active role in emergency drills, tabletop exercises, and interagency meetings (Allen et al., 2010; Trump, 2011). This study exhibits some of the roles that school administration has taken to ensure that emergency drills and school safety meetings take place at their sites.

Darryl, one of the district administrators from RHS \# 3, reported that he conducts weekly safety meetings with school administrators but has monthly meetings with SROs throughout the year to discuss safety issues. Daniel, the district administrator from RHS \#1, is directly involved in safety meetings periodically throughout the school year but primarily relies on the building principals and their SROs to make sure that emergency plans and drills are up to date. Dwight, from RHS \# 2, conducts monthly meetings with the safety liaisons, but also has other duties as a high school assistant principal, which does not leave much time to conduct ongoing meetings with SROs.

All of the principals and SROs in this study typically see each other every school day, but they do not necessarily meet to discuss school security issues. The chasm in the SRO and building principal relationship is not unique to this study. Coon and Travis (2012) argued the importance of having school and law enforcement personnel work together to build school safety 
practices, but the relations between these two groups is not always smooth. However, the interviews of the SROs and principals in this study did demonstrate that these participants have a sincere desire to work collaboratively; it is a matter of making this practice a top priority.

Sharing information and understanding their specific roles and abilities within the school emergency management system is a critical element in relationship building. First responder agencies can provide information about their organization, including their missions, policies, procedures, and limitations, which could be a benefit to the schools (Sinclair et al., 2012). However, this study showed that due to time constraints and educational endeavors, building relationships between principals and SROs is not a priority. This quandary can be resolved by setting aside time for mandatory meetings to discuss specific roles and ideas to develop an effective working relationship.

Dwight provided an analysis of sharing information with his law enforcement counterparts by saying, "Our mission is to teach and keep everyone safe while law enforcement makes sure that we are following the laws. It is important that we work together towards the same objective." Darryl had a different perspective by adding, "We operate independently of each other because of the nature of our jobs, but in the end, it's a uniform approach to service."

The key in this interorganizational relationship is establishing a common objective and what each organization provides in advancing their mutual goal. However, part of the dilemma of the SRO and building principal relationship is the lack of a clear-cut definition of the SRO role as also noted by Coon and Travis (2012). Brown (2006) asserted that the SRO performs an amalgam of educational, correctional, law enforcement, and role modeling tasks. With such a variation of tasks performed by the SRO and with no clear-cut definition of their job, it is arduous to create an alliance with building administrators. 
Samantha, one of the SRO supervisors, described this predicament by noting that building administrators do not fully understand law enforcement policy and procedures and she does not understand theirs. She added, "It takes ongoing, open conversations to avoid miscommunication.” According to Kapucu et al. (2010), building effective relationships between personnel in separate organizations requires ongoing sustainable sessions working together. One of the most effective tools in understanding the role of a counterpart is to study and learn the other's job description, policies, and procedures.

Another facet to the multilayered role of the SRO is the fact that they have multiple supervisors from their respective law enforcement agency and school administration. Rodney described this dynamic by acknowledging that his police department knows that he works for them first, but is placed on assignment to the school. With that in mind, if his police department needs him to respond to an emergency outside of the school, then he must respond accordingly, which may leave the school vulnerable.

Having numerous supervisors creates a dilemma for the SRO when having to make a choice of which lead to follow. Part of the process for building an effective relationship between the building principal and SRO is answering the question of which supervisor, school or law enforcement, takes the lead and under which circumstances. Additionally, having a clear-cut, well defined, agreed upon MOU between the school and law enforcement agency will assist in defining roles, supervision, and protocols in certain events.

MOUs help to alleviate misunderstandings between respective agencies by providing pertinent information in regards to individual responsibilities (Schnobrich-Davis, \& Terrill, 2010). The principal and SRO must gain a thorough understanding of each other's roles, limitations, and abilities before creating mutual objectives. Coon and Travis (2012) purported 
that school administration and law enforcement value school safety, but their differences in cultures and backgrounds could decidedly alter this goal.

In this study, there were a myriad of reasons for some of the shortcomings in developing an effective relationship between the building principal and SRO. For example, Pamela shared the fact that she has had three SROs in a span of six years. The turnover at this position has made it difficult to develop or strengthen this relationship. Likewise, Darryl mentioned the ongoing transitions that have taken place at the school administration level, which creates the same dilemma of having to start over again to build a new relationship. Patricia referred to the problem of having SROs in her district assigned to other schools, which creates a barrier in the communication process. The SRO, which is assigned to her high school, has to leave to handle calls for service elsewhere, which has weakened the level of trust. This is not to say it is the fault of the SRO but it is a hurdle in the relationship. Patricia also reported that SROs look at student issues from a police perspective, but as a building principal, "I must protect the student."

The primary mission of an SRO as a police officer is to uphold the law while a school administrator's mission is to help students towards graduation. Chung (2015) recognized that stakeholders involved in emergency management have distinct roles and responsibilities; however, the importance of overcoming the disparity of each other's roles can make a difference in an effective response. This chasm in the primary missions between the role of a building principal and an SRO has the potential to become troublesome.

Peggy provided a different analysis by asserting that the ability to effectively communicate is simply based on personality differences. She explained that in some cases, it was easier to communicate with the SRO, but in other cases, it was difficult because of their conflicting personalities. In developing organizational relationships, each entity must define 
each other's roles, expectations, missions, styles of operation, capabilities, and limitations (Perry \& Lindell, 2003; Schnobrich-Davis \& Terrill, 2010). Regardless of the reason for varied differences, the building principal must take the lead in developing the relationship with the SRO to provide clarity on how to effectively handle emergency and non-emergency situations as a team.

Creating, implementing, and sustaining school emergency plans require an amalgamation of documents from federal, state, and local areas that are related to emergency management. The process of putting all of this information together to create emergency plans is challenging enough without first having an effective working relationship between schools and law enforcement.

Sub question one focused on the perspective of participants in relation to the creation of school emergency management plans at their respective sites. While each of the schools in this study has comprehensive school safety plans, the creation of the plans is primarily the work of district administrators without the assistance of law enforcement personnel. District administrators are tasked with the responsibility of having school emergency management plans in place that meet the minimum standards created by their respective state educational agencies. Building principals have the responsibility of ensuring their respective school site plans are not only in place, but also maintained through meetings, training, emergency drills, and regular audits. The enormity of establishing school safety is one that cannot be completed without assistance (Cray \& Weller, 2011; Fletcher \& Nicholas, 2015; Trump, 2011).

Assistance in establishing a school safety plan in the three school sites in this study comes from support from law enforcement personnel only during the implementation and sustaining phases of school emergency management plans. According to Roger, when he 
accepted the assignment as an SRO, the written plans were already in place. With his experience as a police officer, he knew what to do in the event of an emergency. When it comes to completing emergency drills, Roger takes a lead role along with his supervisor to coordinate, observe, and conduct after-action meetings.

Rodney had similar sentiments. He divulged that the district administrator takes the lead in creating their school emergency management plans, but as the SRO, he takes the lead role in ensuring that the monthly emergency drills are completed at each of his school sites. Samantha discussed her role in creating school emergency management plans by reporting that the schools typically put the plans together, but "we do step in to help if there are any questions or safety concerns." She added that they act as more a consultant in this process.

An examination of sub question two focused on the perspective of participants in relation to the implementation of school emergency management plans at their respective sites. As argued by Dwyer et al. (2000), school and law enforcement personnel partnerships are important in the development and creation of school emergency plans. Ninety-five percent of administrators reported playing a role in developing school emergency management plans whereas only $64 \%$ of law enforcement have reported contributing to them (Coon \& Travis, 2012). This study showed different results from these two studies.

Pamela talked about not having a hand in developing their school safety plans, but she did have a role in ensuring that the plans were implemented and sustained through emergency drills and exercises. In the other two school sites, the building principal is ultimately responsible for ensuring that school emergency plans are in place, however, the principal delegates the daily responsibility to their assistant principals. The SROs and SRO supervisors discussed similar sentiments by adding that they did not have a role in school safety plan development; however, 
similar to the principals in this study, they do assist with implementation and ongoing sustaining of the school emergency plans. SROs and SRO supervisor's contribution to school emergency management plans in this study come in the form of a support role as an observer, trainer, or consultant.

Daniel reported that the implementation of school emergency management plans is a yearly event in which school and law enforcement personnel have an opportunity to foster their relationship. He said, "SROs go through a checklist with teachers and staff as reminders to that ensure everyone is well versed on the school emergency management plans." Pamela acknowledged that the yearly implementation process is organized by having assistant principals lead safety discussions with faculty and staff. As a group, they discuss the various types of options available during various types of emergencies. Pamela also noted that SROs and SRO supervisors address response methods with faculty and staff regarding various types of emergency events during their regular emergency meetings.

An examination of sub question three focused on the perspective of participants relating to the sustaining of school emergency plans at their respective sites. Participants in this study are in agreement about the importance of having school emergency management plans, but more importantly, the process of sustaining them through regular safety meetings, emergency drills, and training. Having school emergency management plans requires the expertise and assistance from outside agencies as reported by Allen et al. (2010) and O’Toole and Meier (2011) but specifically in collaboration with first-responder agencies as noted by Gerber and Robinson (2009), Hull (2011), and Kano and Bourque (2007).

Darryl from RHS \# 3 mentioned that their annual meetings are held in collaboration with local fire, police, emergency dispatch, the area hospital, and other interested parties, which 
differs slightly than the other school sites in this study. At RHS \# 1, their annual safety meetings are primarily made up of school administrators and law enforcement officers from around the county, whereas RHS\# 2 have safety liaisons from each campus along with their SROs and school administration that attend their annual safety meetings. Sustaining school emergency management plans shows as a collaborative process between school and law enforcement personnel at each of the sites in this study. Daniel added, "We have an SRO at each school site that keeps on top of all emergency drills." Each school site conducts school emergency drills beyond the ones mandated by their respective state along with a debriefing process to discuss the outcomes of the drills.

\section{Theoretical Foundations}

As surmised by McGuire and Silvia (2010), and Reitan (1998), there are multiple conflicting definitions with no clear solutions to provide a framework for analyzing the problems associated with interorganizational relationships. Another description of interorganizational relationships offered by Casey (2007) purported that these types of relationships are close, longterm, mutually-beneficial agreement between two or more partners with shared objectives. McGuire \& Silvia (2010) acknowledged that developing and engaging in effective collaborative networking and relationship building between organizations does not happen by chance: it takes time and hard work. Additionally, many authors believe that the coordination of multiple agencies has been and will continue to be problematic unless the organizations agree to collaborate toward a common goal (Aiken \& Hage, 1968; Coon \& Travis, 2012; Hougland \& Sutton, 1978; Kapucu, 2006a).

Multiple organizations do not have common goals, missions, policies, and interests, which creates a chasm in the relationship (Quarentelli, 1997). Two organizations were the focus 
of this qualitative multiple case study: schools and law enforcement. Each of the school sites in this study exhibits many of the problems associated with multiple organizations working together. For example, Darryl discussed the dilemma of wanting to send an SRO to a training conference, but the SRO's originating agency did not allow it. Dwight noted that school administrators and SROs are not on the same page because they do not attend training together, which creates a lack of collaborative efforts. Daniel offered a unique perspective by adding that the major concern at his site is continual change, whether it is turnover in staff, or changes in policy and procedures between the two organizations. He understands that policies and procedures continually evolve, but these changes make it difficult to establish continuity in creating an effective work environment. Predictably, schools and law enforcement agencies have vastly unique and diverse missions, goals, and objectives, which make it difficult to collaborate to work toward a common goal.

School and law enforcement personnel in this study showed that as part of two separate organizations they do not necessarily have common goals, missions, policies, and interests, which has created a chasm in the relationship. Both principals and SROs reported understanding that they have differing missions and goals. Because of a lack of understanding each other's organizations and roles, an impasse exists, which can be overcome by developing an effective relationship (Chung, 2015; Coon \& Travis, 2012).

One of the most pressing issues in a collaborative relationship involves the coordination of agencies, but it is not a common existence among organizations (Reitan, 1998). Unless the two organizations in this study agree to collaborate toward a common goal, the relationship between them will continue to be problematic. This is not in the best interests of school safety practices. 
In developing organizational relationships, each organization must understand each other's missions, structures, styles of operation, capabilities, and limitations (Perry \& Lindell, 2003; Schnobrich-Davis \& Terrill, 2010). Comments from participants in this study reflect the desire to understand each other's operational capabilities while working toward a collaborative relationship; however, various obstacles exist that deter this from being a possibility.

One of the major obstacles of collaborative opportunities for schools and law enforcement personnel are the priorities of the schools to ensure successful educational opportunities for its students. Schools focus on instructional strategies, educational testing, student attendance, student achievement, and extra-curricular activities, to name a few. These priorities are the responsibility of building principals and administrators who are the key stakeholders in the relationship between school and law enforcement. Given the amount of time needed to ensure school success, which is measured by student performance, there is little or no time to foster relationships outside of educational precedence.

Peggy described a prime example of this dilemma. She reported that she fully understands the importance of having an effective relationship with the SRO, which is crucial, but finding time with all of the educational responsibilities makes it extremely difficult. Stakeholders connected through the emergency management process have distinct roles and responsibilities; however, the seriousness of overcoming the diversity of each other's roles can make the difference in an effective response (Chung, 2015; Coon \& Travis, 2012).

SROs obviously serve a completely different role but clearly understand the importance of collaboration with key stakeholders, namely school administration. Rodney discussed some of his roles as "going from school to school, educating students on everything from drugs to alcohol, cyber-bullying, you name it." At the same time, he understands the predominant need to 
have a strong working relationship with school administration. Rodney also mentioned that one of the biggest barriers in his relationship with the building principal is the lack of time to train together and have meetings.

Sowa (2008) discussed how the interaction between two organizations could effectively progress over time, which could strengthen the trust factor within the relationship. One of the essential steps in developing a collaborative atmosphere between organizations is mutual trust and respect, which is developed through frequent interactions (Ansell \& Gash, 2007; Kapucu et al., 2010; Thomson \& Perry, 2006). Peggy admitted that finding time to collaborate during the school day is difficult because of all her other responsibilities. Nonetheless, it is imperative for these obstacles to be addressed when it comes to the safety of students, staff, and community members. This study shows that time constraints and a myriad of other important objectives in the school setting have created a void in finding time for school administrators to work together with SROs in school emergency management.

From the response of the participants in this study, school emergency management is challenging because most school personnel are not trained to react as first responders (Bobo et al., 2003; Kano \& Bourque, 2007). This plight provides a profound need for schools and law enforcement to establish an effective working relationship. This study clearly shows the need for school and law enforcement personnel to form a trusting, mutually beneficial, and interactive interorganizational relationship. This study provides a unique contribution to the literature on interorganizational relationships by showing the relationship between school and law enforcement personnel at three high schools. 


\section{Implications}

This study was conducted in an attempt to address a gap in the literature regarding interorganizational relationships between schools and law enforcement personnel in the creation, implementation, and sustaining of school emergency management plans. There are a number of studies regarding interorganizational relationships in the context of emergency management and others on the effectiveness of the SRO in schools; however, this qualitative case study is unique in examining the perspective of school and law enforcement personnel in the context of school emergency management.

\section{Theoretical}

According to Kapucu and $\mathrm{Hu}$ (2014), trust and fostering relationships is essential in building an effective emergency management network. Sowa (2008) noted that working and interacting influences how two organizations will effectively progress over time, which could strengthen the trust factor within the interorganizational relationship. The question is if two organizations with diverse cultures, missions, and objectives are able to collaborate to create a comprehensive approach to school emergency management readiness.

One of the major obstacles in establishing an effective relationship between schools and law enforcement personnel, namely the building principal and SRO, is the lack of time to collaborate and work together. Predominate stakeholders, namely, school superintendents and law enforcement command staff must first agree to allow school principals and SROs the necessary time throughout the year to form an effective working relationship. One of the first steps in creating an effective, collaborative atmosphere between organizations is to develop mutual trust and respect through frequent interactions (Ansell \& Gash, 2007; Kapucu et al., 2010; Tomson \& Perry, 2006). School safety is a vital topic for educators but is placed lower on 
the educational priority list because of the mission objectives of our schools. However, community members, parents, and society have a reasonable expectation that students are housed in a safe environment during the school day.

Changeover in the SRO and school administration is also a major problem in developing a sustainable relationship. Pamela, the principal from RHS \# 1 said, "We have had an SRO assigned to our school for years, but now they are rotating every couple of years." She noted that the SRO does not get a chance to know the students and vice-versa because of the constant change over. Darryl stated, "There is continual change in leadership with the law enforcement agency, which requires establishing a new relationship with the incoming police chief. However, change occurs in building administrative staff through turnover, as well, which interferes with building a relationship." Schools and law enforcement should attempt to establish a minimum amount of time assigned to the SRO program, which will assist in establishing a sense of trust and comradery among students and staff.

\section{Empirical}

This study illustrates the need to strengthen the mutual understanding and roles between school and law enforcement personnel. Mission and objectives among educators and law enforcement are varied, which creates a natural divide that can be conquered by using a deliberative and multilateral process in creating an effective interorganizational relationship as argued by Ansell and Gash (2007), and Cray and Weller (2011). Kapucu (2016) posited that sharing information and developing a willingness to collaborate through mutual trust and understanding is critical to developing an effective relationship among differing agencies. It would be beneficial to develop and provide training opportunities for school administration and law enforcement to attend as teams to better each other's roles and developing comradery and 
trust. One type of training opportunity that would be helpful is to develop a course whereby school administration and law enforcement personnel have the opportunity to share their roles, duties, procedures, and limitations. The training could also include mock scenarios that principals and law enforcement have to work through in partnerships, followed by a group debrief after each scenario to share thoughts and ideas.

\section{Practical}

From the results of this study, it is clear that specific duties of the SRO have not been established across school districts in the United States. Some of the roles of the SROs overlap, and each SRO and their respective school site have their own set of duties. This process can cause confusion and a lack of understanding between the two agencies.

One potential solution to clarifying the vague role of the SRO is having state legislators create a formal interpretation of the SRO position (Maranzano, 2001). Having a detailed MOU in place between schools and law enforcement agencies that explains the roles and expectations of each position within the organizations will assist in improving collaborative efforts (Burling \& Hyle, 1997; Cray \& Weller, 2011). Basic information in the MOU should include terms of agreements, sites of assignments, regular duty hours, SRO and building administration training requirements, summer hours, appointment of the SRO, established SRO supervisor (building principal/and/or law enforcement), and termination of the agreement.

Part of the complication in the SRO and building administration partnership is the SRO having supervisors from school and law enforcement. Both organizations must establish a chain of command for the SRO during school sessions and when schools are closed. Furthermore, establishing when and how to release the SRO to handle duties outside of the school when it is in 
session is crucial so the school is not left in a vulnerable state. While having a detailed MOU is a beginning point in addressing this problem, the document must be put into physical practice.

Having an understanding of each other's abilities, policies, procedures, and limitations is critical in creating and maintaining an effective interorganizational partnership. Participants in this study from both school and law enforcement discussed the importance of understanding the other's procedures and limitations in dealing with critical issues. For example, building administrators typically do not have a thorough understanding of criminal and procedural law when it comes to deciding if an arrest is in order. This shortcoming can hinder the relationship between school and law enforcement unless both parties discuss all of the options before becoming involved in a difficult situation. However, there is the possibility of not having the time needed to talk through these situations, which also creates a dilemma.

Structurally, the majority of decisions involving students will be the final decision for the building administrator; however, the student body is only a portion of the overall make-up of persons involved with schools. Each school will have teachers, non-certified staff, parents, and community members that frequent the schools. In cases involving non-students, the premise of the decision falls on the building principal's shoulders, but the SRO may see it from a different perspective. Again, it is imperative that these types of potential problems are discussed through training or meetings with both school administration and law enforcement working together.

The results of this study also indicate that SROs most likely will not understand the entire school disciplinary process and what options school administration can employ when handling student discipline. Student discipline has the distinct possibility of crossover between what is considered a school matter and what is considered a violation of the law. In these cases, it is crucial to discuss which cases will be handled through school discipline or the court system. In 
some cases, school discipline does end up being adjudicated through the juvenile or adult court system. These types of matters may have a tremendous effect on the future of a student, which means that every option should be weighed heavily. On the other hand, school administration must also have a working knowledge of the protocols and limitations of what actions SROs can take in handling student matters. It is impossible to have a contingency plan for every student situation, which makes it crucial to have pre-emptive discussions regarding the decision-making process with school and law enforcement personnel.

\section{Delimitations and Limitations}

Delimitation in this study is the rationale behind the decision to limit or define the focus of this study. In this qualitative multiple case study, this researcher chose three high schools located in school districts located in one rural and two suburban areas in three different states that were similar in the number of high school students served at their respective sites and located schools that employed the use of an SRO from a local law enforcement agency. This researcher's goal was to use only three high schools that were similar to derive analogous information from the participants.

This study was comprised of several limitations. First, the perceptions of the participants from RHS \# 1, RHS \#2, and RHS \#3 may be unique to those high schools, as the working relationship between school and law enforcement personnel are structured uniquely to their specific site. This makes it difficult to make widespread generalizations based on the results of this study.

Participants in this study were purposefully recruited due to fitting the criteria of having an SRO assigned from a local police department to a high school. Perceptions from a middle school that has an SRO assigned to their school may have been different. Additionally, some 
school districts have their own police department, which would have created a different perception of their working relationships of the SROs and building administrators in this study.

This study shows diversity among the participants, which included three male district administrators, two male and one female principal, two male and one female SRO supervisor, and two male and one female SRO. All of the participants had less than five years in their current roles at the time of the interviews; however, most of the participants had between five and 20 years in their respective careers. Perceptions of district administrators, building principals, SROs, and SRO supervisors who have been in their current positions for lengthier periods would most likely be different.

All of the participants in this study provided rich sources of information about school emergency management planning; however, they were not always the person with the most knowledge about the creation, implementation, and sustaining of school emergency management plans. In some interviews, the information provided was based on speculation and could only be validated through other sources of data.

Another limitation of this study is the reliance on participant interviews, documents, and artifacts that pertain to school emergency management planning. The majority of data analysis was focused on my interpretation of transcripts from participant interviews. Participant interview responses were subjective because of each person's individual experiences and perceptions according to their role.

Lastly, given my current position as a director of crisis and safety in a school district and my past experiences as a police officer, $\mathrm{SRO}$, and former building administrator, some researcher bias may have influenced the interpretation of the data. Although this researcher took steps to eliminate possible bias from interfering with individual participant interviews by remaining 
neutral and withholding approval or denial of my opinions during participant responses, it is possible some bias may exist. It was difficult not to respond to participant answers and viewpoints, but this researcher found neutrality to be effective in obtaining open and honest responses from participants during the interview process.

\section{Recommendations for Future Research}

This study examines the overall process of creating, implementing, and sustaining school emergency plans. Each of the 12 participants provided detailed information about this process. Data analysis of this study demonstrates the need for future research on the dynamic between school and law enforcement personnel gaining a thorough understanding of each other's policies, procedures, and limitations in the interagency relationship between schools and law enforcement. It is necessary to understand each other's roles thoroughly in order to make informed decisions regarding school emergency management. It was learned that the building principal and the SRO are the key role players in ensuring safe schools on a daily basis.

A quantitative study with a survey provided to SROs and building principals across the United States in this area could focus on the specific relationship between SROs and building administrators. A quantitative study will provide a multitude of responses based on a Likert scale to gain a general response to the building principal and SRO relationship in a school setting. Along the same lines of inquiry, a quantitative study of school districts that employ SROs through their local law enforcement agency compared with school districts that have their own police department could produce interesting results.

This study shows that some problems with the school and law enforcement partnership suffered in some ways due to dual supervision of SROs through school and law enforcement. Brown (2006) questioned if school and law enforcement relations with two completely different 
cultures can effectively work together on a consistent basis. However, does a school district that employs their own police officers change the cultural dynamic between school and law enforcement personnel?

A multiple case study could focus on state education agencies processes for developing school emergency templates that are provided to each of the school districts. This study indicates there is some reliance based on FEMA's recommendations, but the question remains, to what degree and what agencies assist in developing these templates? Kano and Bourque (2008) reported that approximately $56 \%$ of the schools in the United States have plans that follow federal government school emergency management protocols. Having a school emergency plan template is necessary. However, these types of plans should be developed in collaboration with first-responder agencies that are familiar with the regions and its propensity for their unique environmental hazards (Gerber \& Robinson, 2009; Hull, 2011; Kano \& Bourque, 2007). According to Allen et al. (2010) and O'Toole and Meier (2011), school emergency plans require the expertise and assistance from outside agencies that have a vested interest in the school district.

\section{Summary}

This researcher's experiences in the military, law enforcement, as a D.A.R.E. instructor, SRO, and chief of police, along with time as a high school teacher and building administrator led to questions regarding the effectiveness of the interagency relationship between schools and law enforcement. With the recent rise in school shootings and the potential for natural and technical disasters across the United States, this researcher intended to examine the relationship between school and law enforcement in the hopes of and possibly improving it and potentially saving lives. Many studies have explored the effectiveness of the SRO program and viability in making 
school safer, but few have explored the interagency relationship between school and law enforcement personnel.

It was easy for this researcher to identify with all of the participants in this study having worked in each of the roles of the participants except as an SRO supervisor. This researcher was cognizant of the potential for personal bias during the interview process and data analysis, but the results of this study share significant insight to the current body of literature on school safety and SRO programs. The perceptions and experiences of each of the participants will provide a valuable resource for law enforcement command staff, members of school boards, superintendents, district administrators, building principals, SROs, and SRO supervisors.

School and law enforcement administration must understand the value of having an effective interagency relationship that it takes time, effort, and a willingness to work together toward a common goal. School and law enforcement leaders must also explore the question of whether or not their plans and their relationship are effective. This study showed some of the problems currently associated with relationships between school and law enforcement personnel such as law enforcement not having much input into school emergency management policy. Nonetheless, this researcher witnessed resolve and willingness from the participants to work together to provide a safe haven for students and staff.

The differences associated with school and law enforcement can be overcome by setting aside the time to train and work together toward the goal of having safe schools. While this researcher realizes the goal of schools is to provide an avenue to graduation and prepare students for life after high school and it takes the majority of training and professional development opportunities to improve instruction to ensure student success in passing state mandated exams, school districts must take into consideration the safety of students and staff as well. This means 
that time must be made available for principals and SROs to train together. When parents see their children going to school, they have expectations that their child is going to learn but that they will also return home safely. Policy and decision makers need to realize the need for firstresponders and school staff to work together to develop effective relationships, but that it takes time, planning, and hard work.

The ability of practitioners to develop an effective interorganizational relationship will be enhanced by this study. School and law enforcement leaders should have a better understanding of the need for collaborative efforts to develop an effective relationship with an agreed upon goal of continually improving school safety. Understanding the need to overcome some of the existing barriers such as cultural and mission differences between the two organizations should enhance the possibility of creating an effective partnership. Focusing on the essential aspects of the interorganizational relationships in the context of school emergency readiness should allow other partnerships to be successfully implemented. Taking time to understand and learn each other's role will improve the effectiveness of school emergency management readiness. 


\section{REFERENCES}

Abbasi, A., \& Kapucu, N. (2015). A longitudinal study of evolving networks in response to natural disaster. Computational and Mathematical Organization Theory, 22(1), 47-70. doi:10.1007/s10588-015-9196-7

Agnich, L. E. (2014). A comparative analysis of attempted and completed school-based mass murder attacks. American Journal of Criminal Justice, 40(1), 1-22. doi:10.1007/s12103014-9239-5

Agranoff, R. (1991). Human services integration: Past and present challenges in public administration. Public Administration Review, 51(6), 533. doi:10.2307/976604

Aiken, M., \& Hage, J. (1968). Organizational interdependence and intra-organizational structure. American Sociological Review, 33(6), 912. doi:10.2307/2092683

Allen, P. K., Will, P. K., Brennon, M. D., \& Poirier, M. M. (2010). Preparing public school personnel to effectively respond to a large-scale crisis: Evaluation of an emergency management training. Journal of Emergency Management, 8(2), 21-33. doi:10.5055/jem.2010.0009

American Psychological Association Zero Tolerance Task Force. (2008). Are zero tolerance policies effective in schools? An evidentiary review and recommendations. American Psychologist, 63, 852-862. doi: 10370003-066X.63.9.852

Amsler, L. B. (2016). Collaborative governance: Integrating management, politics, and law. Public Administration Review, 76(5), 700-711. doi:10.1111/puar.12605

Ansell, C., \& Gash, A. (2007). Collaborative governance in theory and practice. Journal of Public Administration Research and Theory, 18(4), 543-571. doi:10.1093/jopart/mum032 
Avery, E. J., Graham, M., \& Park, S. (2016). Planning makes (closer to) perfect: Exploring United States' local government officials' evaluations of crisis management. Journal of Contingencies and Crisis Management, 24(2), 73-81. doi:10.1111/1468-5973.12109

Bhandari, R. B., Owen, C., \& Brooks, B. (2014). Organisational features and their effect on the perceived performance of emergency management organisations. Disaster Prevention and Management: An International Journal, 23(3), 222-242. doi:10.1108/dpm-06-20130101

Blackaby, H. T., \& Blackaby, R. (2011). Spiritual leadership: Moving people on to Gods agenda. Nashville, TN: B \& H Pub. Group.

Blumenstein, L., Fridell, L., \& Jones, S. (2012). The link between traditional police sub-culture and police intimate partner violence. Policing: An International Journal of Police Strategies \& Management, 35(1), 147-164. doi:10.1108/13639511211215496

Bobo, N., Hallenbeck, P., \& Robinson, J. (2003). Recommended minimal emergency equipment and resources for schools: National consensus group report. The Journal of School Nursing, 19(3), 150-156. doi:10.1177/10598405030190030501

Bondü, R., \& Beier, S. (2015). Two of a kind? Differences and similarities of attacks in schools and in institutes of higher education. Journal of Interpersonal Violence, 30(2), 253-271. doi:10.1177/0886260514533156

Bondü, R., \& Scheithauer, H. (2014). Leaking and death-threats by students: A study in German schools. School Psychology International,35(6), 592-608. doi: $10.1177 / 0143034314552346$ 
Borum, R., Cornell, D. G., Modzeleski, W., \& Jimerson, S. R. (2010). What can be done about school shootings? A review of the evidence. Educational Researcher, 39(1), 27-37. doi:10.3102/0013189x09357620

Boulton, M. J., Duke, E., Holman, G., Laxton, E., Nicholas, B., Spells, R., \& Woodmansey, H. (2009). Associations between being bullied, perceptions of safety in classroom and playground, and relationship with teacher among primary school pupils. Educational Studies, 35(3), 255-267. doi:10.1080/03055690802648580

Bradford, E., \& Wilson, M. A. (2013). When terrorists target schools: An exploratory analysis of attacks on educational institutions. Journal of Police and Criminal Psychology, 28(2), 127-138. doi:10.1007/s11896-013-9128-8

Brady, K. P., Balmer, S., \& Phenix, D. (2007). School-police partnership effectiveness in urban schools: An analysis of New York City's impact schools initiative. Education and Urban Society, 39(4) 455-478.

Brouwers, A., \& Tomic, W. (2000). A longitudinal study of teacher burnout and perceived selfefficacy in classroom management. Teacher and Teacher Education, 16(4), 239-253.

Brown, B. (2006). Understanding and assessing school police officers: A conceptual and methodological comment. Journal of Criminal Justice, 34(6), 591-604. doi: 10.1016/j.crimjus.2006.09.013

Brudney, J. L., \& Gazley, B. (2009). Planning to be prepared: An empirical examination of the role of voluntary organizations in county government emergency planning. Public Performance \& Management Review, 32(3), 372-399. doi:10.2753/pmr1530-9576320302 
Burling, W. K., \& Hyle, A. E. (1997). Disaster preparedness planning: Policy and leadership issues. Disaster Prevention and Management: An International Journal, 6(4), 234-244. doi:10.1108/09653569710179075

Burns, W. J., \& Slovic, P. (2007). The diffusion of fear: modeling community response to a terrorist strike. SSRN Electronic Journal. doi:10.2139/ssrn.912736

Casey, M. (2007). Partnership-Success factors of interorganizational relationships. Journal of Nursing Management, 14, 72-83. doi:10.1111/j.1365-2934.2007.00771.x

Chen, C., Yu, K., \& Chen, M. (2012). Planning of professional teacher-training program for disaster prevention education and executing efficiency evaluation. Disaster Prevention and Management: An International Journal, 2(5), 608-623. doi:10.1108/09653561211278734

Cheurprakobkit, S., \& Bartsch, R. A. (2005). Security measures on school crime in Texas middle and high schools. Educational Research,47(2), 235-250. doi:10.1080/00131880500104366

Chikoto, G. L., Sadiq, A., \& Fordyce, E. (2013). Disaster mitigation and preparedness. Nonprofit and Voluntary Sector Quarterly, 42(2), 391-410. doi:10.1177/0899764012452042

Chrusciel, M. M., Wolfe, S., Hansen, J. A., Rojek, J. J., \& Kaminski, R. (2015). Law enforcement executive and principal perspectives on school safety measures: School resource officers and armed school employees. Policing: An International Journal of Police Strategies \& Management, 38(1), 24-39. doi:10.1108/pijpsm-11-2014-0115

Chung, J. (2015). Conflicts and natural disaster management: A comparative study of flood control in the Republic of Korea and the United States. Disasters, 40(3), 554-572. doi:10.1111/disa. 12162 
Clettenberg, S., Gentry, J., Held, M., \& Mock, L. A. (2011). Traumatic loss and natural disaster: A case study of a school-based response to Hurricanes Katrina and Rita. School Psychology International, 32(5), 553-566. doi:10.1177/0143034311402928

Col, J. (2007). Managing disasters: The role of local government. Public Administration Review, 67, 114-124. doi:10.1111/j.1540-6210.2007.00820.x

Comfort, L. K., \& Kapucu, N. (2006). Inter-organizational coordination in extreme events: The World Trade Center attacks, September 11, 2001. Natural Hazards, 39(2), 309-327. doi:10.1007/s11069-006-0030-x

Comfort, L. K., Ko, K., \& Zagorecki, A. (2004, November). Coordination in rapidly evolving disaster response systems: The role of information. American Behavioral Scientist, 48(3), 295-313. Retrieved from http://www.iisis.pitt.edu/publications/Coordination_in_Rapidly_Evolving_Disaster_Resp onse_Systems-2004.pdf

Coon, J. K., \& Travis, L. F. (2012). The role of police in public schools: A comparison of principal and police reports of activities in schools. Police Practice and Research, 13(1), 15-30. doi:10.1080/15614263.2011.589570

Cooper, T. (2013). Empirical research on inter-organizational relations within a national disaster management network in the Caribbean. Public Organization Review, 15(1), 1-16. doi:10.1007/s11115-013-0248-5

COPS Office. (2013). Memorandum of Understanding for School-Based Partnerships. Washington, D.C.: U.S. Department of Justice, Office of Community Oriented Policing Services. https://cops.usdoj.gov/pdf/2014_MOU-FactSheet_v3_092513.pdf 
Cornell, D. G., Gregory, A., \& Fan, X. (2011). Reductions in long-term suspensions following adoption of the Virginia student threat assessment guidelines. NASSP Bulletin,95(3), 175194. doi: $10.1177 / 0192636511415255$

Council of State Governments Justice Center. (2014). School safety plans: A snapshot of legislative action. Retrieved from https://csgjusticecenter.org/wp-content/uploads/2014/03/NCSL-School-Safety-PlansBrief.pdf

Crawford, C., \& Burns, R. (2015). Preventing school violence: Assessing armed guardians, school policy, and context. Policing: An International Journal of Police Strategies \& Management, 38(4), 631-647. doi:10.1108/pijpsm-01-2015-0002

Crawford, C., \& Burns, R. (2016). Reducing school violence: Considering school characteristics the impacts of law enforcement, school security, and environmental factors. Policing: An International Journal of Police Strategies \& Management, 39(3), 455-477. doi:10.1108/pijpsm-05-2016-0061

Cray, M., \& Weller, S. C. (2011). Policy to practice: A look at national and state implementation of school resource officer programs. The Clearing House: A Journal of Educational Strategies, Issues and Ideas, 84(4), 164-170. doi:10.1080/00098655.2011.564987

Creswell, J.W. (2013). Qualitative inquiry \& research design: Choosing among five approaches. ( $3^{\text {rd }}$ ed.). Thousand Oaks, CA: Sage Publications, Inc.

Daniels, J. A., Royster, T. E., Vecchi, G. M., \& Pshenishny, E. E. (2010). Barricaded captive situations in schools: Mitigation and response. Journal of Family Violence, 25(6), 587594. doi:10.1007/s10896-010-9318-4 
Daniels, J. A., Volungis, A., Pshenishny, E., Gandi, G., Winkler, A., Cramer, D., \& Bradley, M. (2009). A qualitative investigation of averted school shooting rampages. Counseling Psychologist, 38(1), 69-95. Retrieved from http://www.wvpsychology.org/wpcontent/uploads/2015/04/Daniels_Volungis_etal_2010. pdf

Department of Homeland Security (DHS). (2013). National response framework, DHS, Washington, D.C. Retrieved from https://www.fema.gov/media.../final_national_response_framework_20130501.pdf

Dillon, R. L., Tinsley, C. H., \& Burns, W. J. (2014). Near-misses and future disaster preparedness. Risk Analysis, 34(10), 1907-1922. doi:10.1111/risa.12209

Dorn, M. S., \& Dorn, C. (2005). Innocent targets: When terrorism comes to school. Macon, GA: Safe Havens International Inc.

Drabek, T. E. (1970). Methodology of studying disasters: Past patterns and future possibilities. American Behavioral Scientist, 13(3), 331-343. doi:10.1177/000276427001300303

Drabek, T. E., \& McEntire, D. A. (2003). Emergent phenomena and the sociology of disaster: Lessons, trends, and opportunities from the research literature. Disaster Prevention and Management: An International Journal, 12(2), 97-112. doi:10.1108/09653560310474214

Dwyer, K. P., Osher, D., \& Hoffman, C. C. (2000). Creating responsive schools: Contextualizing early warning, timely response. Exceptional Children, 66(3), 347-365.

Dynes, R. (1994). Community emergency planning: False assumptions and inappropriate analogies. International Journal of Mass Emergencies and Disasters, 12(2), 141-158. Retrieved from http://www.ijmed.org/articles/430/download/ 
Edmonds, R. R. (1982) Programs of school improvement: An overview. Educational Leadership, 40(3), 4-9.

Fast, L. C., Weaver, J. F., Miller, S. D., \& Ferrin, T. E., Jr. (2016). Training effects on emergency management activation response. Journal of Organizational Psychology, $16(1), 71-77$.

Federal Emergency Management Agency. (2016). Retrieved, from https://www.fema.gov/

Fletcher, J., \& Nicholas, K. (2015). What can school principals do to support students and their learning during and after natural disasters? Educational Review, 68(3), 358-374. doi:10.1080/00131911.2015.1114467

Gerber, B. J., \& Robinson, S. E. (2009). Local government performance and the challenges of regional preparedness for disasters. Public Performance \& Management Review, 32(3), 345-371. doi:10.2753/pmr1530-9576320301

Gereluk, D. T., Donlevy, J. K., \& Thompson, M. B. (2015). Normative considerations in the aftermath of gun violence in schools. Educational Theory, 65(4), 459-474. doi:10.1111/edth.12127

Glickman, C. D., Gordon, S. P., \& Ross-Gordon, J. (2014). Supervision and instructional leadership a developmental approach. Boston, MA: Pearson.

Golway, T. (2003). So others might live: a history of New York's bravest: The FDNY from 1700 to the present. New York, NY: BasicBooks.

Gonzalez, R. A. (2010). Developing a multi-agent system of a crisis response organization. Business Process Management Journal, 16(5), 847-870. doi:10.1108/14637151011076502 
Gulen, E. (2010). Texas law enforcement management and administrative statistics program. Telemasp Bulletin, 17(2).

Heath, M. A., Pamela, K., Dean, B., \& Bingham, R. (2007). History of school safety and psychological first aid for children. Brief Treatment and Crisis Intervention, 7(3), 206223. Retrieved from https://btci.stanford.clockss.org/cgi/content/full/7/3/206/

Homeland Security Presidential Directive 5. (2015). Retrieved June 09, 2017, from https://www.dhs.gov/publication/homeland-security-presidential-directive-5

Hougland, J. G., \& Sutton, W. A. (1978). Factors influencing degree of involvement in interorganizational relationships in a rural county. Rural Sociology, 43(4), 649-670.

Hoy, W. K., \& Sweetland, S. R. (2001). Designing better schools: The meaning and measures enabling school structures. Educational Administration Quarterly, 37(3), 296-321.

Hull, B. (2011). Changing realities in school safety and preparedness. Journal of Business Continuity and Emergency Planning, 5(1), 440-450.

Improving America's Schools Act of 1994, Pub. L. No. 103-382, § 108, Stat. 3518 (1995).

Jackson, A. (2002). Police-school resource officers' and students' perception of the police and offending. Policing: An International Journal of Police Strategies \& Management, 25(3), 631-650. doi:10.1108/13639510210437078.

Jennings, W. G., Khey, D. N., Maskaly, J., \& Donner, C. M. (2011). Evaluating the relationship between law enforcement and school security measures and violent crime in schools. Journal of Police Crisis Negotiations, 11(2), 109-124. doi:10.1080/15332586.2011.581511

Johnson, S. M. (2006). The workplace matters: Teacher quality, retention and effectiveness. Washington, DC: National Education Association. 
Kachel, T. R., Laslo, A. T., \& Nickels, B. (2005). SRO performance evaluation: A guide to getting results. Washington DC: U.S. Department of Justice, Office of Community Oriented Policing Services.

Kano, M., \& Bourque, L. B. (2007). Experiences with and preparedness for emergencies and disasters among public schools in California. NASSP Bulletin, 91(3), 201-218. doi:10.1177/0192636507305102

Kano, M., \& Bourque, L. B. (2008). Correlates of school disaster preparedness: Main effects of funding and coordinator role. Natural Hazards Review, 9(1), 49-59. doi:10.1061/(asce)1527-6988(2008)9:1(49)

Kano, M., Ramirez, M., Ybarra, W. J., Frias, G., \& Bourque, L. B. (2007). Are schools prepared for emergencies? A baseline assessment of emergency preparedness at school sites in three Los Angeles County School Districts. Education and Urban Society, 39(3), 399422. doi: $10.1177 / 0013124506298130$

Kapucu, N. (2006a). Interagency communication networks during emergencies: Boundary spanners in multiagency coordination. The American Review of Public Administration, 36(2), 207-225. doi:10.1177/0275074005280605

Kapucu, N. (2006b). The evolving role of the public sector in managing catastrophic disasters: Lessons learned. Administration \& Society, 38(3), 279-308. doi:10.1177/0095399706289718

Kapucu, N., Arslan, T., \& Demiroz, F. (2010). Collaborative emergency management and national emergency management network. Disaster Prevention and Management: An International Journal, 19(4), 452-468. doi:10.1108/09653561011070376 
Kapucu, N., Garayev, V., \& Wang, X. (2013). Sustaining networks in emergency management. Public Performance \& Management Review, 37(1), 104-133. doi:10.2753/pmr15309576370105

Kapucu, N., \& Hu, Q. (2014). Understanding multiplicity of collaborative emergency management networks. The American Review of Public Administration, 46(4), 399-417. doi:10.1177/0275074014555645

Kapucu, N., \& Sadiq, A. (2016). Disaster policies and governance: Promoting community resilience. Politics and Governance, 4(4), 58-61. doi:10.17645/pag.v4i4.829

Kapucu, N., \& Van Wart, M. (2008). Making Matters Worse. Administration \& Society,40(7), 711-740. doi:10.1177/0095399708323143

Khantzian, E. J., \& Mack, J. E. (1989). Alcoholic's anonymous and contemporary psychodynamic theory. Recent Developments in Alcoholism, 7, 67-89. doi:10.1007/9781-4899-1678-5_4

Lambert, R. D., \& McGinty, D. (2002). Law enforcement officers in schools: setting priorities. Journal of Educational Administration, 40(3), 257-273. doi:10.1108/09578230210427172

Lambert, A. D., \& Steinke, C. M. (2015). Negative perceptions of asking for support in law enforcement: Potential impact on benefit avoidance. International Journal of Police Science \& Management, 17(2), 134-144. doi:10.1177/1461355715583004

Leary, M. R., Kowalski, R. M., Smith, L., \& Phillips, S. (2003). Teasing, rejection, and violence: Case studies of the school shootings. Aggressive Behavior, 29(3), 202-214. doi:10.1002/ab.10061 
Lindell, M. K. (2013). Disaster studies. Current Sociology, 61(5-6), 797-825.

doi:10.1177/0011392113484456

Lindell, M. K., \& Perry, R. W. (2011). The protective action decision model: Theoretical modifications and additional evidence. Risk Analysis, 32(4), 616-632. doi:10.1111/j.1539-6924.2011.01647.x

Madfis, E. (2014). Averting school rampage: Student intervention amid a persistent code of silence. Youth Violence and Juvenile Justice, 12(3), 229-249. doi:10.1177/1541204013497768

Madfis, E. (2016). "It's better to overreact": School officials' fear and perceived risk of rampage attacks and the criminalization of American public schools. Critical Criminology, 24(3), 467-467. doi:10.1007/s10612-016-9323-x

Maranzano, C. (2001). The legal implications of school resource officers in public schools. NASSP Bulletin, 85(621), 76-80. doi:10.1177/019263650108562110

Matsuura, S., \& Shaw, R. (2014). Exploring the possibilities of school-based recovery and community building in Toni District, Kamaishi. Natural Hazards, 75(1), 613-633. doi:10.1007/s11069-014-1344-8

May, D. C., Fessel, S. D., \& Means, S. (2004). Predictors of principals' perceptions of school resource officer effectiveness in Kentucky. American Journal of Criminal Justice, 29(1), 75-93. doi:10.1007/bf02885705

McEntire, D., Souza, J., Collins, M. L., Peters, E. J., \& Sadiq, A. (2012). An introspective glance into damage assessment: Challenges and lessons learned from the Paso Robles (San Simeon) earthquake. Natural Hazards, 61(3), 1389-1409. doi:10.1007/s11069-011-00717 
McDaniel, J. (2001). School resource officers: What we know, what we think we know, what we need to know (Report for the School Safety Strategic Planning Meeting). Raleigh, NC: Center for the Prevention of School Violence, North Carolina Department of Juvenile Justice and Delinquency Prevention. Retrieved from http://www.ncdjjdp.org/

McGuire, M. (2006). Intergovernmental management: A view from the bottom. Public Administration Review, 66(5), 677-679.

McGuire, M., \& Silvia, C. (2010). The effect of problem severity, managerial and organizational capacity, and agency structure on intergovernmental collaboration: Evidence from local emergency management. Public Administration Review, 70(2), 279-288. doi:10.1111/j.1540-6210.2010.02134.x

Meyer, H. A., \& Astor, R. A. (2002). Child and parent perspectives on routes to and from school in high crime neighborhoods. Journal of School Violence, 1(4), 101-128. doi:10.1300/j202v01n04_07

Mitchell, R. M., Kensler, L., \& Tschannen-Moran, M. (2016). Student trust in teachers and student perceptions of safety: positive predictors of student identification with school. International Journal of Leadership in Education, 21(2), 135-154. doi:10.1080/13603124.2016.1157211

Myrstol, B. A. (2011). Public perceptions of school resource officer (SRO) programs. Western Criminology Review, 12(3), 20-40.

National Association of School Resource Officers. (n.d.). Retrieved from https://nasro.org/frequently-asked-questions/ 
Newman, K., \& Fox, C. (2009). Repeat tragedy: Rampage shootings in American high school and college settings, 2002-2008. American Behavioral Scientist, 52(9), 1286-1308. doi:10.1177/0002764209332546

NYSAFE Act Gun Reform. (n.d.). safeact.ny.gov. Retrieved from https://safeact.ny.gov/

O’Toole, L. J., \& Meier, K. J. (2011). Public management in interdependent settings: Networks, managerial networking, and performance. Retrieved from http://citeseerx.ist.psu.edu/viewdoc/download?doi=10.1.1.596.9821\&rep=rep1\&type=pdf

O’Toole, M. E. (2000). The school shooter: A threat assessment perspective. Quantico, VA: Critical Incidence Response Group, National Center of the Analysis of Violent Crime, FBI Academy. Retrieved from http://www.fbi.gov/stats-services/publications/shool-shooter

Paoline, E. A. (2003). Taking stock: Toward a richer understanding of police culture. Journal of Criminal Justice, 31(3), 199-214. doi:10.1016/s0047-2352(03)00002-3

Perrow, C. (2011). The next catastrophe: reducing our vulnerabilities to natural, industrial, and terrorist disasters. Princeton, NJ: Princeton University Press.

Perry, R. W., \& Lindell, M. K. (2003). Preparedness for emergency response: Guidelines for the emergency planning process. Disasters, 27(4), 336-350. doi:10.1111/j.03613666.2003.00237.x

Perumean-Chaney, S. E., \& Sutton, L. M. (2012). Students and perceived school safety: The impact of school security measures. American Journal of Criminal Justice, 8(4), 570-588. doi:10.1007/s12103-012-9182-2 
Price, J. H., Khubchandani, J., Payton, E., \& Thompson, A. (2015). Reducing the risks of firearm violence in high schools: Principals' perceptions and practices. Journal of Community Health,41(2), 234-243. doi:10.1007/s10900-015-0087-0

Provan, K.G., \& Milward, H.B. (1995). A preliminary theory of interorganizational network effectiveness: A comparative study of four community mental health systems. Administrative Science Quarterly, 401(1), 1, doi: 10.111/033-3352.

Provan, K. G., \& Milward, H. B. (2001). Do networks really work? A framework for evaluating public-sector organizational networks. Public Administration Review, 61(4), 414-423. doi:10.1111/0033-3352.00045

Quarantelli, E. (1993). Community crises: An exploratory comparison of the characteristics and consequences of disasters and riots. Journal of Contingencies and Crisis Management, 1(2), 67-78. doi:10.1111/j.1468-5973.1993.tb00009.x

Quarantelli, E. (1997). Ten criteria for evaluating the management of community disasters. Disasters, 21(1), 39-56. doi:10.1111/1467-7717.00043

Randozzo, M. R., \& Cameron, J. K. (2012). From presidential protection to campus security: A brief history of threat assessment in North American schools and colleges. Journal of College Student Psychotherapy, 26(4), 277-290. doi:10.1080/87568225.2012.711146

Reitan, T. C. (1998). Theories of interorganizational relations in the human services. Social Service Review, 72(3), 285-309. doi:10.1086/515760

Rocque, M. (2012). Exploring school rampage shootings: Research, theory, and policy. The Social Science Journal, 49(3), 304-313. doi:10.1016/j.soscij.2011.11.001

Roher, E. M., \& Warner, M.A. (2006) Emergency response planning in schools. Education Law Journal, 16(1), 30-59. 
Sadiq, A., Tharp, K., \& Graham, J. D. (2016). FEMA versus local governments: Influence and reliance in disaster preparedness. Natural Hazards, 82(1), 123-138. doi:10.1007/s11069016-2183-6

Schnobrich-Davis, J., \& Terrill, W. (2010). Interagency collaboration. Policing: An International Journal of Police Strategies \& Management, 33(3), 506-530. doi:10.1108/13639511011066881

Seyle, D. C., Widyatmoko, C., \& Silver, R. C. (2013). Coping with natural disasters in Yogyakarta, Indonesia: A study of elementary school teachers. School Psychology International,34(4), 387-404. doi:10.1177/0143034312446889

Shaw, R., Shiwaku, K., \& Sakurai, A. (2016). Disaster resilience of education systems: Experiences from Japan. Tokyo, JP: Springer.

Shiwaku, K. (2014). Comparative study on teacher training for school disaster management in Armenia and Japan. Disaster Prevention and Management: An International Journal, 23(2), 197-211. doi:10.1108/dpm-12-2012-0144

Shiwaku, K., Sakurai, A., \& Shaw, R. (2016). Disaster resilience of education systems: Experiences from Japan. Tokyo, JP: Springer.

Sampson, Z. (2012). Five years after the Virginia Tech massacre, colleges gauge threats. Huffington Post. Retrieved from http://www.huffingtonpost.com/2012/04/14/5years-after-the-virgini_n_1425749.html

Sinclair, H., Doyle, E. E., Johnston, D. M., \& Paton, D. (2012). Assessing emergency management training and exercises. Disaster Prevention and Management: An International Journal, 21(4), 507-521. doi:10.1108/09653561211256198 
Sindhi, S., (2013). Creating safe school environment: Role of school principals. The Tibet Journal. 38(1-2), 77-89.

Sowa, J. E. (2008). Implementing Interagency Collaborations. Administration \& Society,40(3), 298-323. doi:10.1177/0095399707313701

Stake, R. E. (2006). Multiple case study analysis. New York: The Guilford Press.

Steelman, T. A., \& Mandell, M. (2011). Understanding what can be accomplished through interorganizational innovations: The importance of typologies, context and management strategies. SSRN Electronic Journal. doi:10.2139/ssrn.1931076

Stinson, P. M., \& Watson, A. M. (2014). The nature of crime by school resource officers: Implications for SRO programs. SAGE Open, 4(1). doi:10.1177/2158244014521821

Taugher, T. (n.d.). New York, NY fire drill causes panic, Sept 1882. GenDisasters.com. Retrieved from https://www.gendisasters.com/new-york/13698/new-york-ny-fire-drillcauses-panic-sept-1882

The long, shameful list of school shootings in America. (2017). Retrieved from https://everytownresearch.org/school-shootings/

Thomson, A. M., \& Perry, J. L. (2006). Collaboration processes: Inside the black box. Public Administration Review, 66, 20. Retrieved from http://ezproxy.liberty.edu/login?url=https://search-proquestcom.ezproxy.liberty.edu/docview/197177786?accountid=12085

Trump, K. S. (2011). Proactive school security and emergency preparedness planning. Thousand Oaks, CA: Corwin. 
U.S. Department of Education, Office of Elementary and Secondary Education, Office of Safe and Healthy Students. (2013). Guide for developing high quality school emergency operations plans. Retrieved from https://rems.ed.gov/docs/REMS_K-12_Guide_508.pdf

Wang, J. (2016). Study on the context of school-based disaster management. International Journal of Disaster Risk Reduction, 19, 224-234. doi:10.1016/j.ijdrr.2016.08.005

Watson, P. G., Loffredo, V. J., \& Mckee, J. C. (2011). When a natural disaster occurs: Lessons learned in meeting students' needs. Journal of Professional Nursing, 27(6), 362-369. doi:10.1016/j.profnurs.2011.09.001

Waugh, W. L. (2003). Terrorism, homeland security and the national emergency management network. Public Organization Review, 3(4), 373-385. doi:10.1023/b:porj.0000004815.29497.e5

Waugh, W. L., \& Streib, G. (2006). Collaboration and leadership for effective emergency management. Public Administration Review, 66(S1), 131-140. doi:10.1111/j.15406210.2006.00673.x

Watson, P. G., Loffredo, V. J., \& Mckee, J. C. (2011). When a natural disaster occurs: Lessons learned in meeting students' needs. Journal of Professional Nursing, 27(6), 362-369. doi:10.1016/j.profnurs.2011.09.001

Weinstein, N. D. (1989). Effects of personal experience on self-protective behavior. Psychological Bulletin, 105(1), 31-50. doi:10.1037//0033-2909.105.1.31

Wolfe, S. E., Chrusciel, M. M., Rojek, J., Hansen, J. A., \& Kaminski, R. J. (2017). Procedural justice, legitimacy, and school principals' evaluations of school resource officers. Criminal Justice Policy Review, 28(2), 107-138. doi:10.1177/0887403415573565 
Yin, R. K. (1989). Case study research: design and methods. Thousand Oaks, CA: Sage.

Yin, R. K. (2014). Case study research: design and methods. London, UK: Sage Publication. 


\section{APPENDIX A \\ INTERVIEW QUESTIONS}

Interview Questions

1. Please introduce yourself, length of time in your current position and length of time in your career.

2. Describe your working relationship with others (e.g., SRO, SRO supervisor, principal, district level participant)?

3. Discuss the process used to create the current written school emergency plan.

4. Describe the process that was used to implement the school emergency plan.

5. Discuss the training methods used to sustain school emergency management readiness.

6. Describe the barriers that affect the interagency relationship with others (e.g., SRO, SRO supervisor, principal, district level participant) in the context of school emergency management

7. What influences affect the interagency relationship with others (e.g., SRO, SRO supervisor, principal, district level participant) in the context of school emergency management

8. How do the mission objectives for your school affect the interagency relationship with school and law enforcement personnel in school emergency management?

9. What do you believe are the strengths of your working relationship with others (e.g., SRO, SRO supervisor, principal, district level participant) in the context of school emergency management?

10. What do you believe are the weaknesses of your working relationship with others (e.g., SRO, SRO supervisor, principal, district level participant) in the context of school emergency management?

11. How do you foster the relationship with others (e.g., SRO, SRO supervisor, principal, district level participant) in the context of school emergency management

12. Describe the process used to come to a consensus with others (e.g., SRO, SRO supervisor, principal, district level participant) in the context of school emergency management.

13. Describe the process used to examine and assess written plans, training, and the 
ability to effectively respond to an emergency event involving the school.

14. What steps can be taken to improve the interagency relationship between school and law enforcement personnel? 


\title{
APPENDIX B
}

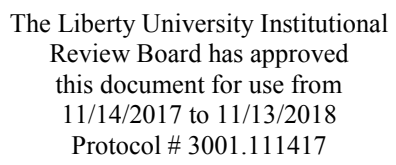

\author{
CONSENT FORM \\ A MULTIPLE CASE STUDY OF THE INTERAGENCY RELATIONSHIP BETWEEN \\ SCHOOL ADMINISTRATORS AND LAW ENFORCEMENT PERSONNEL IN THE \\ CREATION, IMPLEMENTATION, AND SUSTAINING OF SCHOOL EMERGENCY \\ MANAGEMENT PLANS
}

\author{
Robert Lopez \\ Liberty University \\ School of Education
}

You are invited to be in a research study that is designed to analyze the interagency relationships and emergency school management plans that are used to protect students and staff members in the event of a manmade, natural, or technical disaster on or near a school campus. As an educator and parent, my hope and motivation is that this study will contribute to school emergency management readiness, which could potentially save lives.

Please read this form and ask any questions you may have before agreeing to be in the study. Robert Lopez, a doctoral candidate in the School of Education at Liberty University, is conducting this study.

Background Information: School campuses, both pre-k-12 and post-secondary institutions, are vulnerable to a multitude of potential problems including terrorist attacks, natural, and technical disasters. You were selected as a possible participant because your school district and/or law enforcement agency has agreed to participate in this qualitative multiple case study.

The purpose of this study is to investigate how school and law enforcement personnel in the Mid-West, South, and Southwest regions of the United States with diverse cultures, missions, and objectives collaborate to create, implement, and sustain school emergency management readiness.

Procedures: If you agree to be in this study, I would ask you to do the following things:

1. Allow the researcher in this case, Robert Lopez, to conduct an interview that will take anywhere from an hour to an hour and half that will be audio recorded for transcription purposes.

2. Allow the researcher to examine or make copies of documents that are related to this study. For example, school safety plans, safety meeting minutes, maps of buildings, MOU's, exercise or drill(s), and job descriptions as allowed and applicable.

Risks and Benefits of Participation: The risks involved in this study are minimal, which means they are equal to the risks you would encounter in everyday life. There are no direct benefits to participating in this study.

Benefits to society include an additional qualitative examination of school safety practices and school emergency management. It is hopeful that this study will add to the current literature concerning school emergency management.

Compensation: Participants will not be compensated for participating in this study. 


\section{APPENDIX C \\ LIBERTY UNIVERSITY. \\ INSTITUTIONAL REVIEW BOARD}

November 14, 2017

Robert Lopez

IRB Approval 3001.111417: A Multiple Case Study of the Interagency Relationship between School Administrators and Law Enforcement Personnel in the Creation, Implementation, and Sustaining of School Emergency Management Plans

Dear Robert Lopez,

We are pleased to inform you that your study has been approved by the Liberty University IRB. This approval is extended to you for one year from the date provided above with your protocol number. If data collection proceeds past one year, or if you make changes in the methodology as it pertains to human subjects, you must submit an appropriate update form to the IRB. The forms for these cases were attached to your approval email.

Thank you for your cooperation with the IRB, and we wish you well with your research project.

Sincerely,

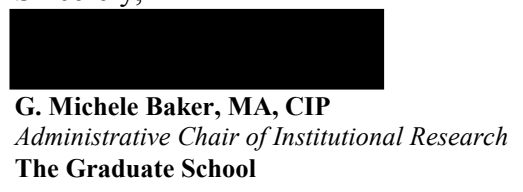

\section{LIBERTY}

U N I V E R I T Y .

Liberty University | Training Champions for Christ since 1971 


\section{APPENDIX D}

\begin{tabular}{|c|c|c|}
\hline \multicolumn{3}{|c|}{ Source Codes - I-Interviews, D - Documents, A - Artifacts, LR - Literature Review } \\
\hline Code & Number & Source \\
\hline After-Action & 2 & I, D \\
\hline Assessment & 14 & I, D \\
\hline Attrition & 10 & $\mathrm{I}$ \\
\hline Barriers & 12 & I, D, LR \\
\hline Collaboration & 20 & I, LR \\
\hline Communication & 74 & I, D, LR \\
\hline Consensus & 11 & I, LR \\
\hline Demographics & 12 & $\mathrm{I}$ \\
\hline FEMA/State & 26 & $I, D, A, L R$ \\
\hline Foster & 12 & I, LR \\
\hline Improved Relations & 13 & I, LR \\
\hline Influences & 12 & 1 \\
\hline Lack of Training & 3 & I, LR \\
\hline Mission Objectives & 19 & I, D, LR \\
\hline $\mathrm{MOU}$ & 5 & I, A, LR \\
\hline Mutual Understanding & 31 & I, LR \\
\hline Networking & 43 & I, LR \\
\hline NIMS/ICS & 10 & I, D, A, LR \\
\hline Priorities & 21 & $\mathrm{I}$ \\
\hline Role & 27 & I, LR \\
\hline Strengths & 13 & 1 \\
\hline Time & 29 & $\mathrm{I}$ \\
\hline Training & $\overline{54}$ & I, D, A, LR \\
\hline Trust & 10 & I, LR \\
\hline Weaknesses & 13 & 1 \\
\hline Written Plans Creation & 13 & $\mathrm{I}, \mathrm{D}, \mathrm{A}, \mathrm{LR}$ \\
\hline $\begin{array}{l}\text { Written Plans } \\
\text { Implementation }\end{array}$ & 11 & $\mathrm{I}, \mathrm{D}, \mathrm{A}, \mathrm{LR}$ \\
\hline Written Plans Sustaining & 10 & $\mathrm{I}, \mathrm{D}, \mathrm{A}, \mathrm{LR}$ \\
\hline
\end{tabular}


APPENDIX E

\begin{tabular}{l|l|}
\hline Codes & Themes \\
\hline $\begin{array}{l}\text { After-Action, Assessment, Collaboration, } \\
\text { Communication, Creation of Written Plans, } \\
\text { Implementation of Written Plans }\end{array}$ & Communication \\
\hline $\begin{array}{l}\text { Barriers, Demographics, Foster, Improved } \\
\text { Relations, Strength, Trust, Weakness }\end{array}$ & Networking \\
\hline Consensus, Mission Objectives, MOU, Role & Mutual Understanding \\
\hline Influences, Time & Priorities \\
\hline Attrition, FEMA/State, Lack of Training, \\
NIMS/ICS, Sustaining Written Plans, Time
\end{tabular}

